\title{
Numerical modelling of single- and multi-phase flow and transport processes in porous media for assessing hydraulic fracturing impacts on groundwater resources
}

\author{
Dissertation \\ zur Erlangung des mathematisch-naturwissenschaftlichen Doktorgrades \\ "Doctor rerum naturalium" \\ der Georg-August-Universität Göttingen \\ im Promotionsprogramm Geowissenschaften \\ der Georg-August University School of Science (GAUSS) \\ vorgelegt von
}

Reza Taher Dang Koo aus Ahvaz, Iran

Göttingen 2020 
Betreuungsausschuss

Prof. Dr. Martin Sauter

Georg-August-Universität Göttingen

Dr. Alexandru Tatomir

Uppsala University

Mitglieder der Prüfungskommission

Referent Prof. Dr. Martin Sauter

Georg-August-Universität Göttingen

Korreferenten Prof. Dr. Holger Class

Universität Stuttgart

Weitere Mitglieder der Prüfungskommission

Dr. Alexandru Tatomir

Uppsala University

Prof. Dr. Thomas Ptak-Fix

Georg-August-Universität Göttingen

Prof. Dr. Jonas Kley

Georg-August-Universität Göttingen

Prof. Dr. Volker Thiel

Georg-August-Universität Göttingen

Tag der mündlichen Prüfung: 19.05.2020 



\section{Abstract}

The environmental footprint of hydraulic fracturing has gained substantial attention in the water - energy nexus as the technology is relatively new and its long-term impacts are not thoroughly understood. Of primary concerns are the injections of large volumes of chemicallaced water into underground and possible upward migration of chemicals and hydrocarbons to groundwater systems. The current active debates within the scientific community are the environmental risks versus reward ratio of the operation. To date, different modelling and observational studies have explored the contamination of drinking water resources from fracturing operations.

This dissertation investigates migration of fracturing and formation fluids from gas formations towards shallow groundwater by means of numerical modelling. First, a generic features, events and processes (FEP) database is used to identify the most relevant factors to define failure scenarios for safety and risk assessment. Out of various scenarios, the focus is on (i) fracturing fluid and brine migration along a conductive fault over the injection and shut-in periods, (ii) fracturing fluid and brine migration along a leaky abandoned well over the lifetime of a typical horizontal well and (iii) methane migration from a natural gas well through overburden sediments. Single and two-phase two-component flow and transport models are employed for the numerical modelling purposes. The spatial and temporal behavior of the contaminant plume in the subsurface, the solute concentration and the arrival times to the aquifer are assessed. Sensitivity analysis are performed to understand the relative importance of key parameters (e.g. hydrogeological parameters) on the flow and transport of contaminants to the shallow aquifer.

The results showed that the contamination probability of shallow aquifers by the upward migration of fracturing fluid and brine from a deep gas formation is low. It was observed that only a limited amount of fracturing fluid could reach the aquifer in a long-term period under specific conditions, such as the presence of a permeable pathway. The hydrodynamic properties of the permeable pathway and its distance from the operation were the most important factors controlling the flow of fracturing fluid to the aquifer. Moreover, well 
production and dilution of fracturing fluid during the transport reduce the rise of fluids to the aquifer in the long-term.

Methane is more likely to migrate upward to shallower strata compared to fracturing fluid and deep brine due to the strong buoyancy. Time to breakthrough and flow rates of methane to groundwater monitoring wells strongly depend on the integrity and distribution of lowpermeability rocks with respect to the leaky natural gas well. Methane can be manifested in groundwater monitoring wells even at distances of larger than $1 \mathrm{~km}$ from the source of leakage because of the flow deviation along low-permeability rocks. The presence of tilted features could further explain fast-developing methane contamination and large lateral spreading reported in field studies. The shape of the contaminant plume in the subsurface, the arrival time to groundwater (if at all) can vary based on hydrogeological characteristics of formations intercalated between the aquifer and gas reservoir. 


\section{Acknowledgments}

I would like to express my thanks and appreciation to my very first teachers, my parents, for all the great lessons throughout my life. Since childhood, I have been fortunate to learn from an exceptionally supportive and kind network of teachers. I owe all of you a great debt of gratitude and I cannot thank you enough for what you have done for me.

Special recognition should go to my advisor Prof. Martin Sauter for the tremendous support during the program. I would like to thank you for giving me the opportunity to be part of an interesting research and for constructive and critical discussions, which greatly helped me to find my $\mathrm{PhD}$ path. Thanks for providing the funding assistance and making it possible to focus only on the research.

I am grateful to my advisor Dr. Alexandru Tatomir for mentoring me during the last four years. I would like to take the time to express the deepest appreciation for your time, patience, encouragement, and scientific support. I could come to your office whenever I faced difficulties in my work, and your suggestions always helped me to find the solution. I was lucky to work with you Alex and thanks for being more than an advisor.

I would like to extend my appreciation to the examination board Prof. Holger Class, Prof. Thomas Ptak, Prof. Jonas Kley and Prof. Volker Thiel. Thanks to my friends and colleagues at the department, especially Iulia, Bettina, Marco, Quan and Xintao. Thanks to Marco for taking the time and effort to review the first version of the dissertation. I appreciate the research assistant job offered by Prof. Jonas Kley and Dr. Elco Luijendijk from the Structural Geology and Geodynamics Department. Thanks to Elco for the insightful comments and suggestions. I had the chance to share the office with wonderful colleagues Viet, Pengxian and Monthon that later become my friends. We shared many happy moments.

Finally, I wish to register a huge vote of thanks to my family, in particular my parents, for their love, support and encouragement. Words are powerless to express my feeling and thanks to my family as it was not possible to finish this $\mathrm{PhD}$ without their support. To them, I dedicate this dissertation. 


\section{Table of Contents}

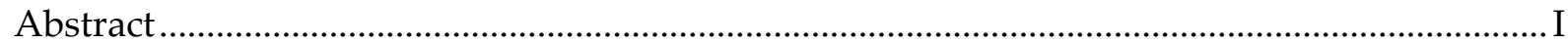

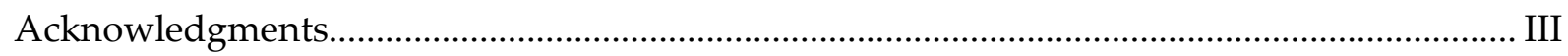

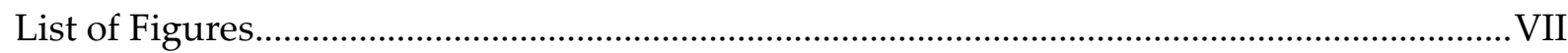

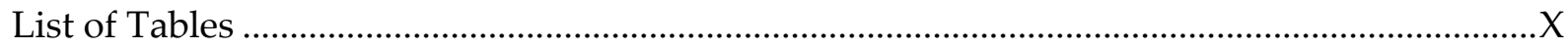

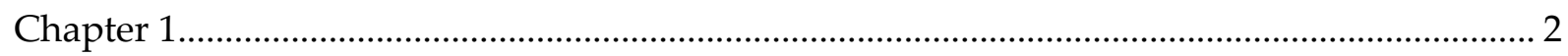

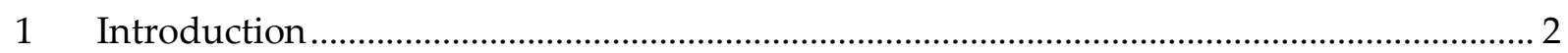

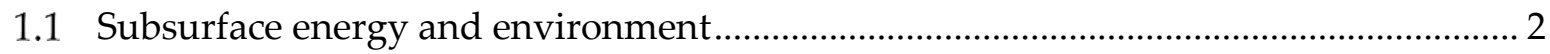

1.2 Contaminant migration to shallow groundwater.......................................................... 5

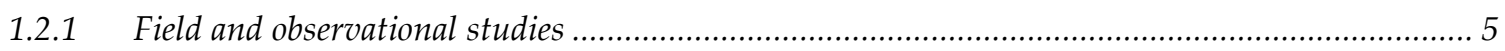

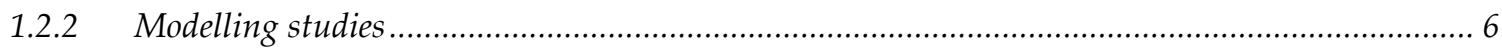

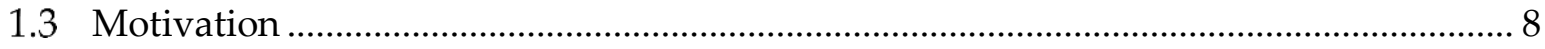

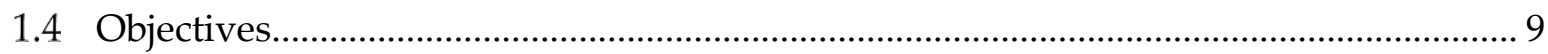

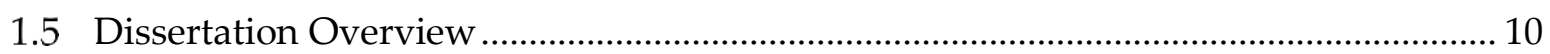

Chapter 2

2 Conceptual model development using a generic features, events and processes (FEP)

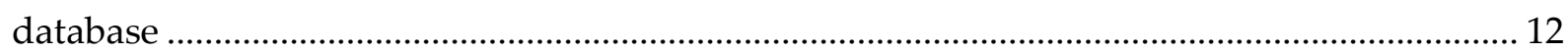

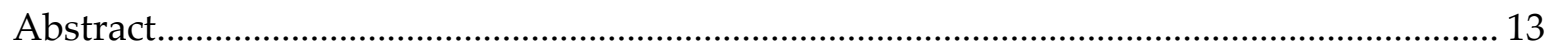

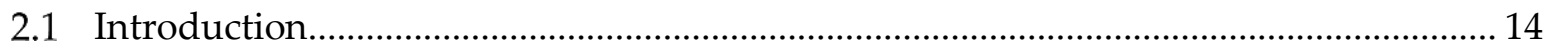

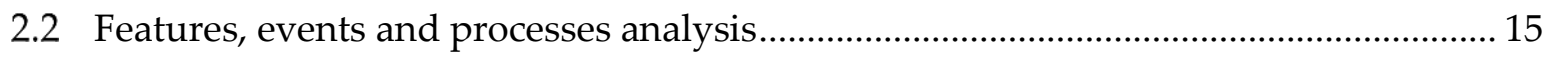

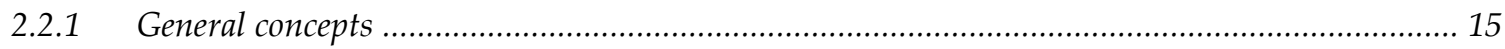

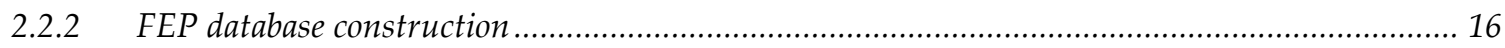

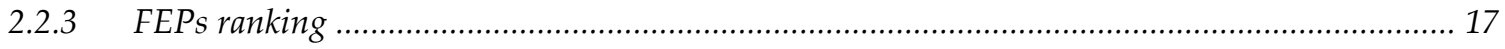

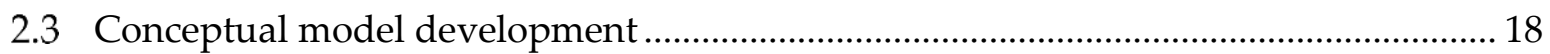

2.4 Regional-scale stray gas migration conceptual model .................................................. 20

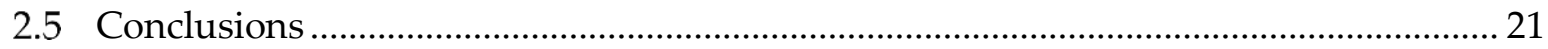

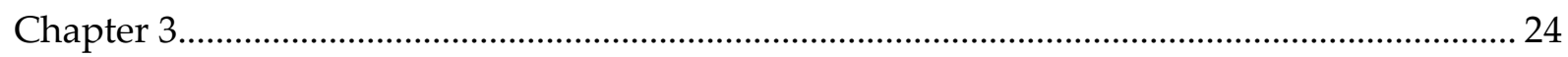

3 Fracturing fluid migration along a fault zone ................................................................ 24

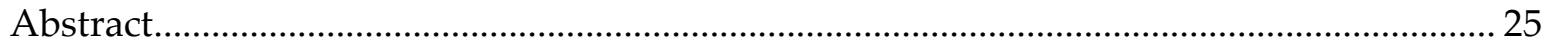




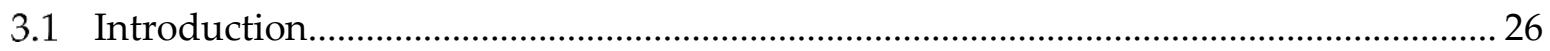

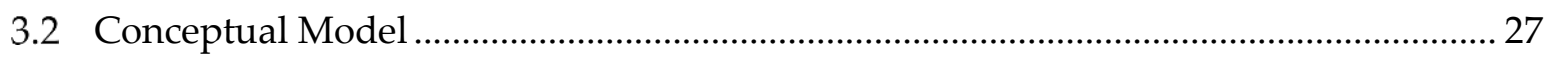

3.3 Mathematical and numerical model ............................................................................... 31

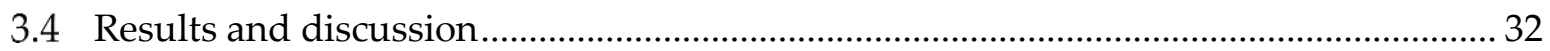

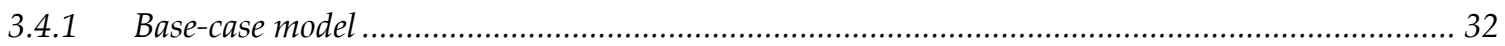

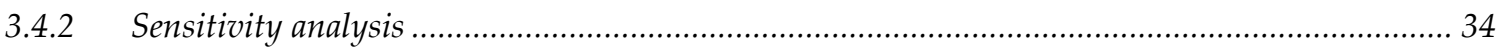

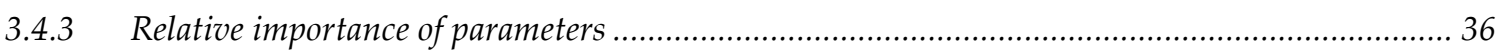

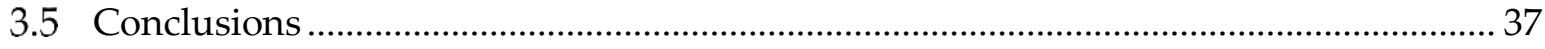

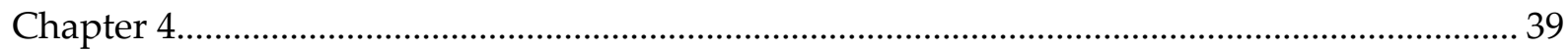

$4 \quad$ Fracturing fluid migration along an abandoned well .................................................... 39

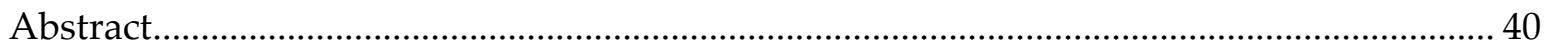

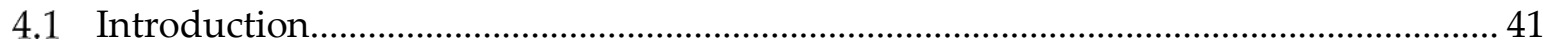

4.2 The North German Basin......................................................................................... 43

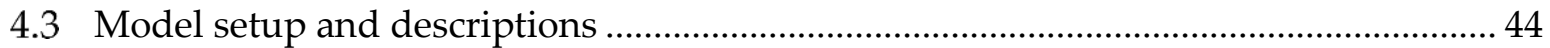

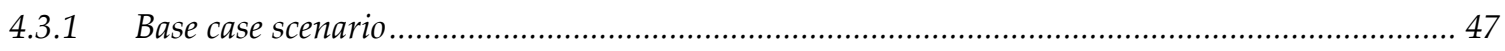

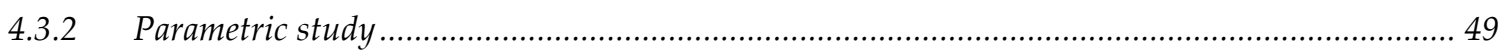

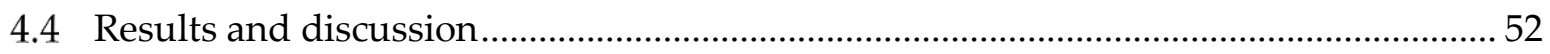

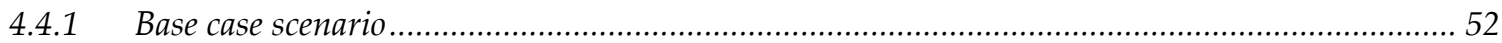

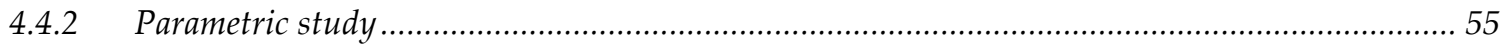

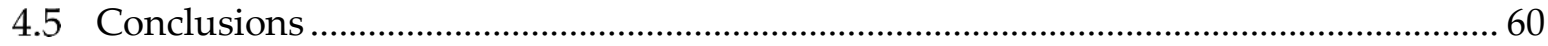

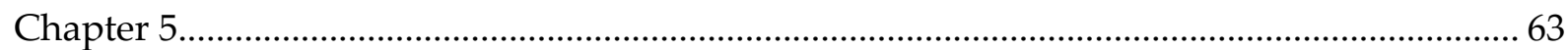

$5 \quad$ NAR neural networks to predict fracturing fluid leakage into shallow groundwater.... 63

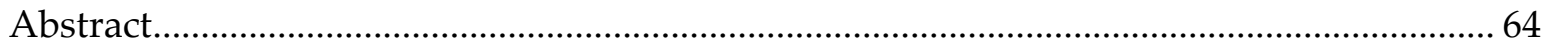

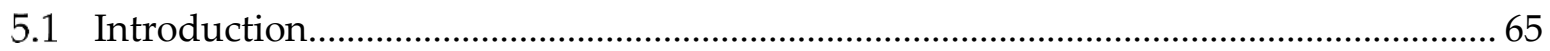

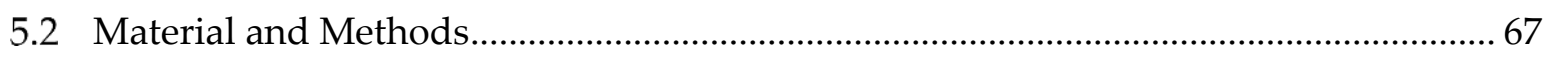

5.2.1 Conceptual model for fracturing fluid migration along an abandoned well ................................ 67

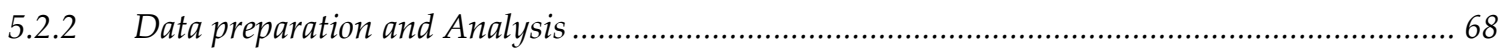

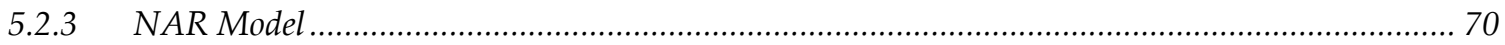

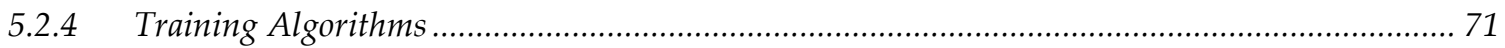

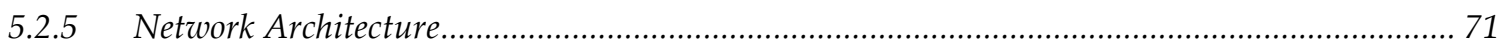

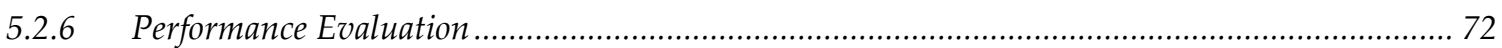


5.3 Results and Discussion

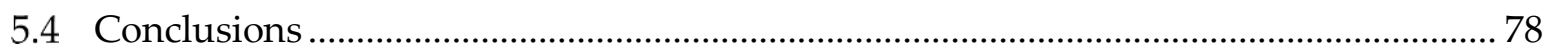

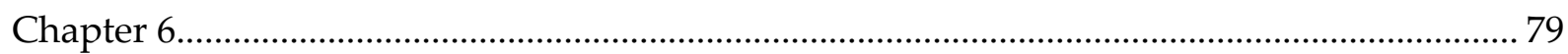

6 Methane migration through overburden sediments …...................................................... 79

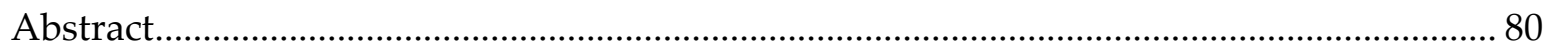

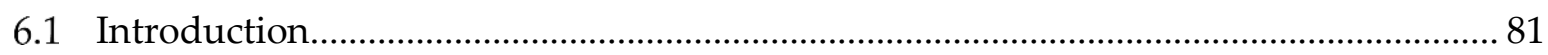

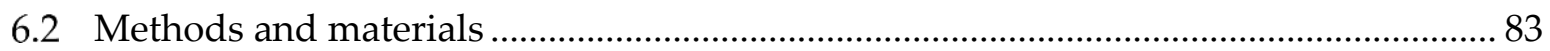

6.2.1 Conceptual model for methane and brine migration at the basin scale ...................................... 83

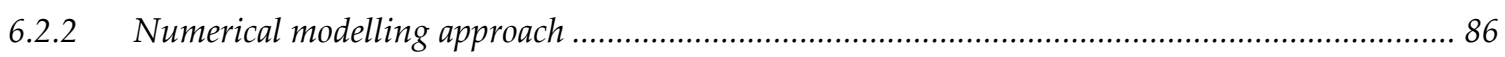

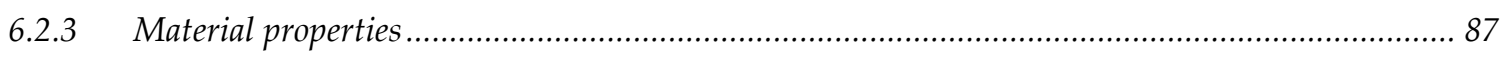

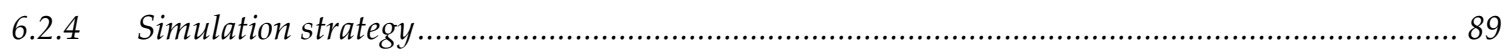

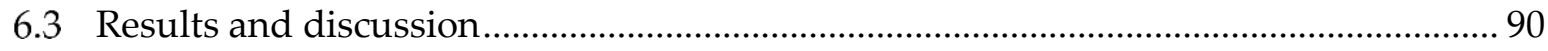

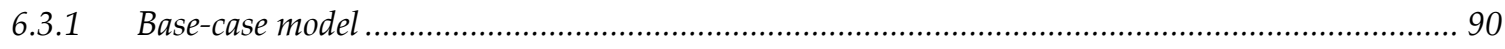

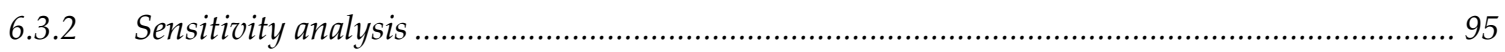

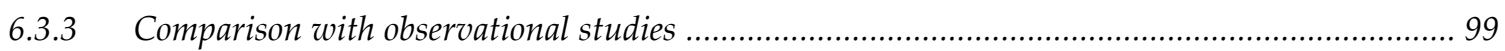

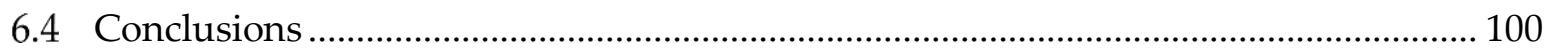

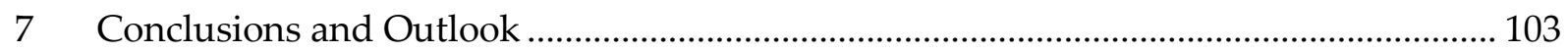

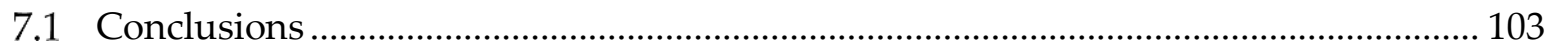

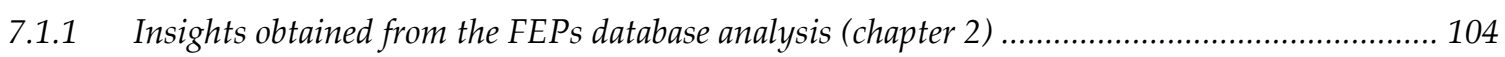

7.1.2 Insights obtained from fracturing fluid migration along a fault zone (chapter 3) ...................... 104

7.1.3 Insights obtained from fracturing fluid migration along an abandoned well (chapter 4) .......... 105

7.1.4 Insights obtained from application of a NAR model to predict fracturing fluid leakage rate...... 106

7.1.5 Insights obtained from methane migration through overburden rocks (chapter 6).................... 106

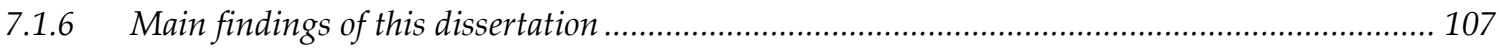

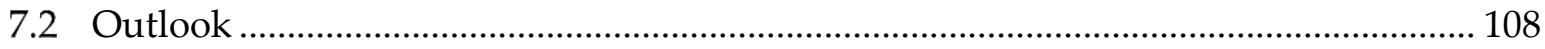

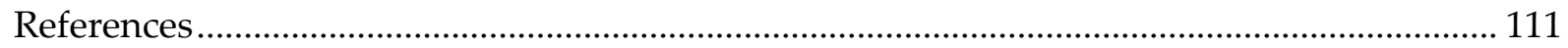




\section{List of Figures}

Figure 1-1. Timeline of activities in unconventional gas development.

Figure 1-2. Six modelling scenarios defined in FracRisk project (Tatomir et al., 2018, 2016). 9

Figure 2-1. The six focused scenarios for the combined evaluation of FEPs. S1: hydraulic fracturing processes; S2: fluid injection, fluid migration and micro-seismics; S3: Source characterization, produced fluid and remaining fluid; S4: short-term flow and transport through fault zones and abandoned wells; S5: long-term regional transport of fluids; S6: long-term diffusive transport through overburden layers and aquifers (source: http://www.fracrisk.eu, Christopher Mcdermott).

Figure 2-2. The main FEPs categories in environmental impact assessment of hydraulic fracturing for shale gas extraction.

Figure 2-3. General workflow for the construction of the conceptual models.

Figure 2-4. Regional scale conceptual model for migration of methane and fracturing fluids.

Figure 3-1. (a) A cross section schematic of a fault zone intersecting the stimulated reservoir volume (SRV) in a hydrocarbon bearing formation and the conceptual representation of the source (HCBF) - pathway (conductive fault) - receptor (aquifer). (b) FS1: with considering the HCBF. (c) FS2: without considering the $\mathrm{HCBF}$.

Figure 3-2. (a) Domain discretization for the numerical simulation. (b) fracturing fluid concentration profile at the end of shut-in period (FS1; sub-scenario: open). 33

Figure 3-3. Fracturing fluid concentration breakthrough curves throughout injection and shut-in period, observed at the aquifer base. (a) FS1. (b) FS2. 34

Figure 3-4. FS1 (model considering the presence of HCBF): (a-h) fracturing fluid concentration in the aquifer at the end of the injection period for each investigated parameter range; (i) pressure profile along the fault zone for four "base-case" sub-scenarios. 35

Figure 3-5. FS2 (model not including the presence of HCBF): (a-h) fracturing fluid concentration in the aquifer at the end of the injection period for each investigated parameter range; (i) pressure profile along the fault zone for four "base-case" sub-scenarios. 36

Figure 3-6. The ranked impacts of the key parameters on the upward migration of fracturing fluid along a fault towards the aquifer: (a) parameter importance of the individual sub-scenarios (equation 34); (b) the calculated mean value of parameter importance of all sub-scenarios (equation 3-5) for the each investigated parameter. 37

Figure 4-1. A schematic of the hydraulic fracturing intersecting an abandoned well 43

Figure 4-2. The conceptual model setup with local resolution at the fracture-well interface. 47 
Figure 4-3. The distribution of fracturing fluid in the $y-z$ plane at $x=1250 \mathrm{~m}$ at the end of (a) injection;

(b) shut-in period.

Figure 4-4. The concentration of fracturing fluid dissolved chemicals along the abandoned well in the $\mathrm{y}-\mathrm{z}$ plane at the end of (a) injection and shut-in periods; (b) first production, second production and post-production periods. 53

Figure 4-5. Fracturing fluid flow rate along the abandoned well at the overburden-aquifer interface during the simulation time

Figure 4-6. Spatial distribution of fracturing fluid along the abandoned well and its neighboring area at the end of (a) first production; (b) second production; (c) post-production period. The cut plane is located at $\mathrm{y}-\mathrm{z}$ direction at $x=1250 \mathrm{~m}$.

Figure 4-7. Fracturing fluid flow rate along the abandoned well at the overburden-aquifer interface during the entire simulation time; (a) injection volume; (b) shale permeability; (c) shale porosity; (d) shale overpressure gradient; (e) overburden thickness; (f) overburden permeability; (g) salinity gradient; (h) abandoned well permeability; (i) fracture-well proximity; (j) and scenario without production.

Figure 5-1. The conceptual model used for simulations. 68

Figure 5-2. Fracturing fluid flow rate to the aquifer for the base-case model during the simulation time.

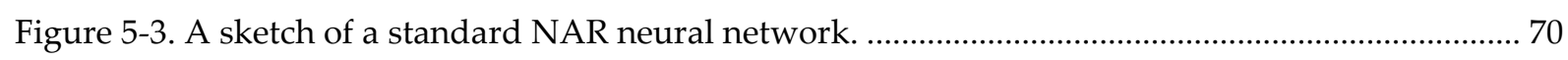

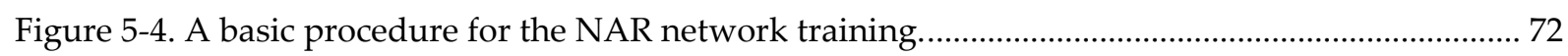

Figure 5-5. Error autocorrelations of (a) NAR-LM and (b) NAR-BR models for the training phase...... 74

Figure 5-6. Performance of the NAR-LM model for the prediction of fracturing fluid flow rate to the aquifer. (a) Training and (b) Testing. 75

Figure 5-7. Performance of the NAR-BR model for the prediction of fracturing fluid flow rate to the aquifer. (a) Training and (b) testing. 75

Figure 5-8. Convergence plots of the NAR models in terms of number of epochs. (a) NAR-LM and (b) NAR-BR 76

Figure 5-9. Response of (a) NAR-LM and (b) NAR-BR models in predicting fracturing fluid flow rate to the shallow aquifer. The top panels show modeled fluid flow rates of the base-case values in Table 5-1. The bottom panels display the model's performance error in training, validation and testing phases. 77

Figure 6-1. Generalized conceptual model of methane and brine migration from the gas reservoir into the overlying formations for the base-case model. The low-permeability layer, i.e. clay, is indicated in gray and permeable sediments in light brown. The dark dashed lines represent the monitoring 
locations at various distances to the leaky gas well. The leakage area is presented in yellow. The boundary conditions employed in the simulations are shown.

Figure 6-2. The distribution of (a) permeability and (b) porosity in the domain. 89

Figure 6-3. Gas phase saturation profiles after (a) 7 days and (b) 2 years of methane leakage into the overburden formations. This model assumes miscible flow and transport of fluids. 91

Figure 6-4. Pressure profiles after (a) 7 days and (b) 2 years of methane leakage into the overburden formations. This model assumes miscible flow and transport of fluids.

Figure 6-5. Spatial distribution of (a) gas phase saturation and (b) dissolved methane (mg L-1) in the aqueous phase after 25 years of methane leakage into the overburden formations. The white dashed lines show the monitoring locations.

Figure 6-6. Gas phase saturation profiles after (a) 7 days and (b) 2 years of methane leakage into the overburden formations. This model assumes immiscible flow and transport of fluids. 94

Figure 6-7. Pressure profiles after (a) 7 days and (b) 2 years of methane leakage into the overburden formations. This model assumes immiscible flow and transport of fluids. 94

Figure 6-8. (a) Methane and (b) brine flow rates at 2000, 3000, $4000 \mathrm{~m}$ distances from the leaky gas well for immiscible and miscible flow models. 94

Figure 6-9. Methane flow rates at 2000 and $3000 \mathrm{~m}$ distances from the leaky gas well for the miscible flow model varying: (a) entry pressure, (b) pore size distribution index, (c) residual water saturation, (d) tilt of formation, (e) methane inflow rate and (f) methane inflow rate in a formation with $1^{\mathrm{o}}$ tilt. 97

Figure 6-10. Gas phase saturation profiles after 15 years of methane leakage into the overburden. The influence of: (a) clay layer integrity and different multi-layered clay systems ((b) case I and (c) case II) are being analyzed. The top panels show the geometry of the clay layers at shallow depths. . 99 Figure 6-11. Measured methane flow into shallow groundwater for different geometries of clay layers. 


\section{List of Tables}

Table 2-1. The highest ranked features for the six focused scenarios. ................................................. 21

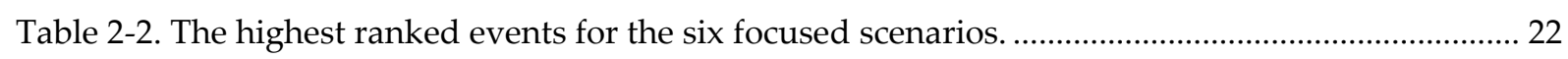

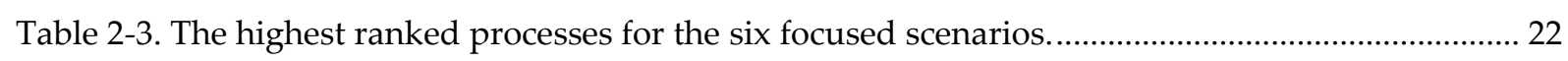

Table 3-1. Parameters used for base case models and sensitivity analysis. .......................................... 29

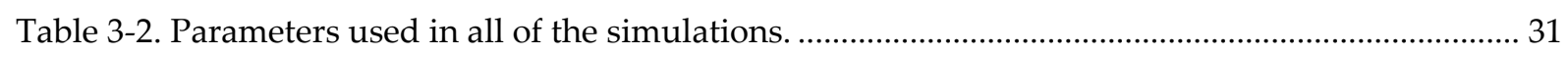

Table 4-1. Summary of basic parameters used in the model (Pfunt et al., 2016).................................. 44

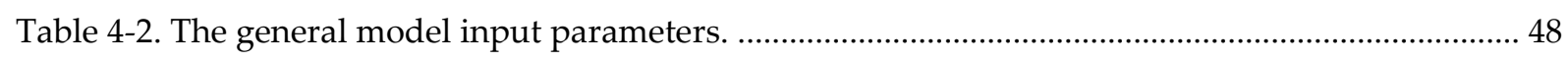

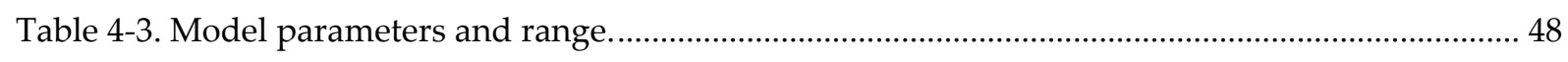

Table 5-1. Parameters used in base-case model and sensitivity analysis simulations.............................69

Table 5-2. Evaluation of the NAR-LM and NAR-BR models performances........................................... 74

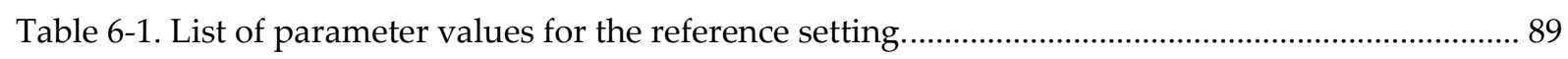




\section{Chapter 1}

\section{Introduction}

\subsection{Subsurface energy and environment}

The global demand for energy is continuously increasing around the world and the International Energy Agency (IEA) anticipated more than 25\% growth by 2040 (IEA (International Energy Agency), 2018). The natural gas extraction from shale and tight gas reservoirs has shown a promising future for energy supply as a bridge fuel toward a lowcarbon energy system (Brown et al., 2009; McGlade et al., 2013). Natural gas will continue to shake up the market and will be the second largest fuel in the global energy mix in 2030 (IEA (International Energy Agency), 2018).

The economical production from unconventional resources is challenging, as the hydrocarbon is stored within ultra-low permeability and low porosity rock formations. An effective well stimulation practice increases the ability to recover hydrocarbon by enhancing local permeability by creating fracture networks within the reservoir (King, 2012). Hydraulic fracturing is commonly applied in unconventional oil and gas extraction and along with horizontal drilling, has significantly contributed to the surge in hydrocarbon production. Most of all new wells drilled and completed today are hydraulically fractured horizontal wells (Cook et al., 2018). A summary of general activities at a fracturing site is shown in Figure 1-1 (U.S. EPA, 2015).

A fracturing job is usually performed in multiple stages with isolating small segments of the wellbore at a time. A large amount of pressurized fluids is then injected into the formation to crack the rock. Fracturing fluid is mainly composed of water (98 - 99\% of total volume) with chemicals including friction reducer, disinfectant, surfactants, gelation chemicals, scale inhibitor, corrosion inhibitor and hydrochloric acid. The fracturing fluid carries proppant (usually sand or ceramic particles) into the newly developed fractures to keep them open following the release of hydraulic pressure (Ge and Ghassemi, 2011; King, 2012; Wang et al., 2016). After fracturing, the pressure ceases and some of the fracturing fluid along with oil and gas from the reservoir flow back to the surface. 
A single fracturing stage may last between 20 minutes to 4 hours and up to 12 fractures can be created. The fractures are narrow, e.g. usually have between 2 to $3 \mathrm{~mm}$ width (King, 2012), with a maximum height of $600 \mathrm{~m}$ above well perforations (Flewelling et al., 2013). Fracture height growth can be easily restricted with the presence of barrier rocks and fracturing fluid leak-off from the fractures into the surrounding porous media (Wang et al., 2018). The evidences of fracture height limit are provided by micro-seismic analysis, fluid tracers, tilt meters, pressure tests and logging data (Fisher and Warpinski, 2012; King, 2012; Weng et al., 2011).

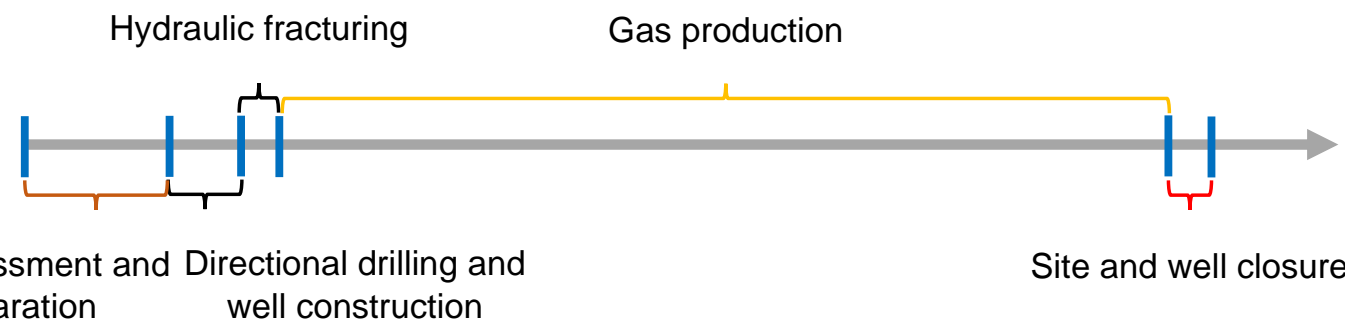

Figure 1-1. Timeline of activities in unconventional gas development.

The surge in unconventional gas development has been projected to have environmental impacts on surface water and groundwater resources. The most frequent and severe threats are more likely from water withdrawals for drilling and hydraulic fracturing, discharge of inadequately treated wastewater, leaks and spills of fracturing fluid chemicals or produced water and migration of fracturing and formation fluids from the reservoir into groundwater resources (Lange et al., 2013; Zhang and Yang, 2015). In the following, these threats are briefly reviewed to better understand the potential impacts to water resources.

The water withdrawal can be from surface water or groundwater (Freyman, 2014), which may become vulnerable to depletion under long-term unsustainable use for hydraulic fracturing (Kondash et al., 2018). The World Resources Institute estimated that $40 \%$ of countries with large shale resources face high levels of water stress or arid conditions (Reig et al., 2014). The risk of water stress is low in humid regions, but local stress could occur in streams and groundwater under high water consumption during a fracturing job, particularly during droughts and high water demands (Barth-Naftilan et al., 2015; Entrekin et al., 2018). The recycle and reuse of fracturing fluid could contribute in reducing the water withdrawal from 
freshwater resources (Chen et al., 2014). Rosa et al. (2018) suggested that water consumption for shale development is dwarfed by human activities, but it would compete with the local activities and environmental water needs in water stressed areas.

Flowback water, i.e. a mixture of fracturing fluid and formation water, turns to the surface within several days following a fracturing job (King, 2012; Nicot and Scanlon, 2012). The produced water, i.e. formation water, flows to the surface over the lifetime of the well (Shih et al., 2015). Generally, the wastewater (flowback and produced water) contains aromatic hydrocarbons, heavy metals, radioactive materials and solvents (Butkovskyi et al., 2017). The wastewater can be disposed by reinjection into the subsurface through deep-well injection, recycle for the subsequent fracturing operation and treated before disposal into surface waters (Annevelink et al., 2016; Butkovskyi et al., 2017; O’Donnell et al., 2018). The partial treatment and reuse of wastewater is the preferred method, where feasible. Otherwise, advanced treatments should be incorporated to satisfy the requirements for surface disposal (Sun et al., 2019).

The spill and leakage of fluids and chemicals could occur on the well pads and during the transportation to and from the site (Clancy et al., 2018; EPA, 2015; McLaughlin et al., 2016). The spill data from unconventional oil and gas wells between 2005 and 2014 in Colorado, New Mexico, North Dakota and Pennsylvania suggested that between 2 and $16 \%$ of wells faced a spill per annum. The common type of hydraulic fracturing-related spill is flowback water (EPA, 2015) and the most common causes are equipment failure and human error (Clancy et al., 2018; EPA, 2015). Moreover, reports indicated a significant increase in the number of truck accidents near fracturing sites (Blair et al., 2018; Muehlenbachs and Krupnick, 2013), which could lead to the release of fluids to the environment (Eshleman and Elmore, 2013; Muehlenbachs and Krupnick, 2013). The leakage and spill of flowback and produced water pose environmental threats to water resources and soil ecosystems because of their elevated salt content, organic additives, metals/metalloids and radioactive elements (Chen et al., 2017; Goodman et al., 2016).

The migration of fracturing and formation fluids from hydrocarbon formations could potentially contaminate shallow groundwater (Darrah et al., 2014; Jackson et al., 2013; Kissinger et al., 2013; Osborn et al., 2011; Rice et al., 2018a). Natural and anthropogenic 
permeable pathways such as leaky oil and gas abandoned wells, fault zones and extensive fracture systems could facilitate the vertical movement of fluids to groundwater (Brownlow et al., 2016; Gassiat et al., 2013; Kissinger et al., 2013). Upward migration of methane to overlying layers is substantially faster than fracturing fluids and brine, given the high buoyancy of natural gas (Jackson et al., 2013; Kissinger et al., 2013; Osborn et al., 2011; Reagan et al., 2015). Fracturing fluid and brine may reach to shallow aquifers through preferential pathways, especially if the reservoir is buried at shallow depth or in sedimentary basins with high overburden permeability (Birdsell et al., 2015a; Gassiat et al., 2013; Wilson et al., 2017).

\subsection{Contaminant migration to shallow groundwater}

\subsubsection{Field and observational studies}

The origin of contaminants in stream flows and groundwater can be typically revealed through geological, hydrological and geochemical analysis (Darrah et al., 2015; Jackson et al., 2013; Siegel et al., 2015). Naturally occurring methane in groundwater is from biogenic or thermogenic processes. Biogenic methane is bacterially produced and sourced from in-situ fermentation or reduction of carbon dioxide. Thermogenic methane, on the other hand, is associated with the upward migration from deep hydrocarbon frmations over geological time (Darrah et al., 2015; Gorody, 2012). Moreover, thermogenic methane may reach groundwater system through anthropogenic activities (Darrah et al., 2015; Warner et al., 2012).

Osborn et al. (2011) and Jackson et al. (2013) conducted compositional and isotopic analyses on water samples from water wells in Pennsylvania and New York and found methane in $80 \%$ of the wells. They observed elevated levels of methane concentration in the water wells within $1 \mathrm{~km}$ distance to oil and gas activities. Osborn et al. (2011) found no proof of shallow aquifer contamination from fracturing fluid and brine migration from the deep subsurface. Later Molofsky et al. (2013) tested a large number of water samples from water wells in northeastern Pennsylvania and found a lack of regional relation between methane concentration in groundwater and distance to oil and gas activities. They identified that the dissolved methane is best correlated with topography and groundwater geochemistry, suggesting thermogenic methane is naturally migrated upward from the hydrocarbon formation. These findings were inconsistent with Jackson et al. (2013) and Osborn et al. (2011) studies. 
Sherwood et al. (2016) analyzed water samples from 924 water wells in the Wattenberg Field of Colorado and found dissolved methane in 593 wells. Methane from 42 water wells had thermogenic origin, which was attributed to oil and gas activities. Nicot et al. (2017) and Wen et al. (2016) examined the origin of organic compounds in groundwater within the Barnett Shale and found a lack of relationship between dissolved methane and proximity to gas wells. They suggested that methane certainly has a natural origin. Harkness et al. (2017) suggested both biogenic and thermogenic methane in shallow groundwater in northwestern West Virginia are unrelated to fracturing. Botner et al. (2018) examined groundwater in the Utica Shale of Ohio and found no relation between methane concentration or source and proximity to gas wells. It is manifested that the contamination impact to groundwater resources varies strongly within individual basins and between basins.

\subsubsection{Modelling studies}

Myers (2012b) was first to develop a numerical model to explore the upward migration of fracturing fluid following hydraulic fracturing. He modeled fluid flow along a vertical fault to the shallow aquifer in the Marcellus Shale. Other studies criticized this model because of the unrealistic geology and assumptions (Cohen et al., 2013; Flewelling and Sharma, 2014; Saiers and Barth, 2012). Addressing the critiques, Myers highlighted that the sensitivity analyses (Myers, 2012a) is used to explores the contaminant transport through various lithologies described for the region (Myers, 2012b). Kissinger et al. (2013) focused on the modelling of methane, fracturing fluids and brine migration in the Münsterland Cretaceous basin and the Lower Saxony Basin, Germany. They suggested that upward migration of free-phase methane and a small amount of liquid-phase fluids is plausible in case there is a connective permeable pathway. More recent studies employing more complex geological models suggested that fracturing fluid could not reach groundwater aquifers without the presence of connective pathways such as fractures, faults and abandoned wells (Birdsell et al., 2015a; Gassiat et al., 2013; Schwartz, 2015). Flewelling \& Sharma (2014) suggested that the vertical movement of fracturing fluid and brine is more often constrained by low permeability of overburden rocks and lack of a driving force.

Birdsell et al. (2015) performed simulations to explore fracturing fluid migration through the lifetime of a horizontal well in the Marcellus Shale. They found that well production reduces the contamination threat to shallow aquifers. Brownlow et al. (2016) explored fracturing fluid 
flow along abandoned wells in the Eagle Ford Shale play, Texas. They suggested that oil and gas wells converted into water wells pose higher contamination threats to shallow groundwater. Other studies suggested that fracturing fluid does not extend to groundwater aquifers, even with the presence of permeable pathways (Edwards et al., 2017; Pfunt et al., 2016). Evaluating fracturing fluid migration in the North German Basin, Pfunt et al. (2016) found that the fracturing fluid is diluted to minor concentrations during the long-term transport. Edwards et al. (2017) suggested that fracturing fluid is imbibed into the source rock and retained there for a long period. Multiple studies have highlighted the importance of geological and hydrogeological characteristics of sedimentary basins on the vertical movement of fracturing fluid.

Methane contamination of shallow aquifer is more likely comparing to fracturing fluids and brine (Kissinger et al., 2013; Osborn et al., 2011; Reagan et al., 2015; Schwartz, 2015). Kissinger et al. (2013) modeled methane transport from the source rock through overburden formations over 100 years at the field scale. They suggested that methane leakage to shallow groundwater is plausible under specific conditions, such as the presence of a connecting permeable pathway, low residual gas saturation in the pathway and release of large volumes of methane from the source rock. Later Reagan et al. (2015) performed simulations on methane and brine migration to aquifers over a 2 year period, and compared fluid migration through faults or fractures with pre-existing gas wells. It was observed that the transport through a well annuli convey more methane to overlying layers than fractures and faults, because of lower void spaces of connecting features in the well. They suggested that methane leakage to the aquifer is more likely to be transient. Afterward, several studies focused on methane migration from the source rock towards shallow groundwater through the wellbore, as the main pathway for methane transport (Nowamooz et al., 2015; Rice et al., 2018b).

Nowamooz et al. (2015) explored methane and brine movement along the casing of a decommissioned well in the St. Lawrence Lowlands, Quebec. They suggested that hydrodynamic properties of the casing annulus are the most important factors controlling methane arrival time to the aquifer. Their results showed that in most cases methane flow to the aquifer is sustained over the simulation time (100 years), which is inconsistent with Reagan et al. (2015) findings. This is due to large differences in the applied conceptual models and assumptions, such as neglecting overpressure in the reservoir in Reagan et al. (2015). A recent 
study, Rice et al. (2018b), investigated the influence of multiphase parameters on methane transport from a gas well into the shallow aquifer in the Pierre Shale in Northeastern Colorado. The study suggested that parameters affecting capillarity and relative permeability have strong influence on flow rates and volumes of methane reaching the aquifer. Moortgat et al. (2018) simulated methane transport from a faulty gas well into fractured and unfractured aquifers and compared high-pressure with continues slow gas leakage. They suggested that pulses of high-pressure leakage into fractured media are required for observing rapid and extensive lateral spreading of methane.

\subsection{Motivation}

The FracRisk project was initiated under Horizon 2020 program LCE-16-2014 to develop a knowledge base for understanding, preventing and mitigating the environmental impacts of shale gas exploration and exploitation, and to provide scientific-based yet practical recommendations to minimize the potential impacts (https://www.fracrisk.eu). Six modelling scenarios (Figure 1-2) are defined under the scope of the project (Tatomir et al., 2018, 2016). Scenario 1 focuses on the coupled multiphase flow and geomechanical processes related to hydraulic fracturing. Scenario 2 focuses on the short-term response of geological settings to fracturing fluid injection into the source rock. Scenario 3 simulates the reactive transport of fracturing fluid through the induced fractures within the source rock during the fracturing operation and flowback period. Scenario 4 deals with the short-term flow and transport of fracturing fluid and methane through pre-existing pathways such as fault zones and abandoned wells. Scenario 5 focuses on the regional flow and long-term migration of fracturing fluid and methane in the subsurface. Scenarios 6 deals with the long-term diffusive transport of methane through overlying formations and groundwater systems (Tatomir et al., 2018, 2016). 


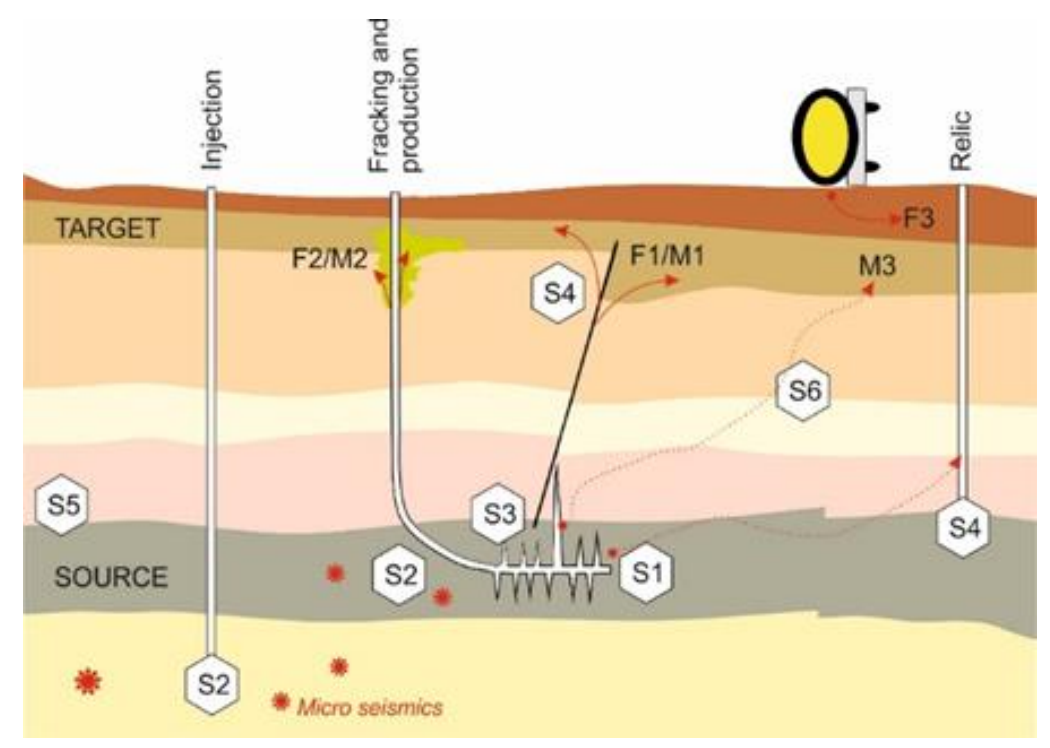

Figure 1-2. Six modelling scenarios defined in FracRisk project (Tatomir et al., 2018, 2016).

This dissertation is defined under the scope of scenarios 4 and 5 to study probable failure scenarios leading to the potential migration of contaminants to overlying layers. Multiple studies linked groundwater contamination with hydrocarbon development and it is imperative to examine the scope of the problem under various conditions. The extent of the contamination plume in the subsurface and its evolution over time should be studied. The role of natural and anthropogenic pathways in the transport of contaminants requires further attention. The relative importance of key parameters and processes on the upward migration of fluids needs to be investigated. The impetus for this research is the need to develop numerical models to analyze the risk of groundwater contamination by upward migration of fracturing and formation fluids via various permeable pathways.

\subsection{Objectives}

This doctoral dissertation focuses on modelling of fracturing fluid, methane, and brine migration from deep hydrocarbon formations into shallow aquifers following hydraulic fracturing. Hypothetical failure scenarios are designed and simulated correspondingly to scrutinize the potential contamination hazard to shallow groundwater. The scenarios are borrowed from real cases reported in the oil and gas industry. In this regard, commercial and open-source numerical simulators are used for modelling single and multiphase flow and transport processes in fractured and porous media. The detailed objectives are as follows: 
1- Using a generic FEP database in determining the most relevant failure scenarios in the context of hydraulic fracturing. In the next step, defining and developing conceptual models to assess the long-term impacts.

2- Investigating the migration of fracturing fluid and brine from hydrocarbon formations towards the aquifer to ensure the quality of drinking water resources remain intact after the operation.

3- Investigating the influence of pre-existing permeable pathways namely, fault zones and abandoned wells on the vertical migration of fracturing fluid and brine to shallow aquifers during various phases of hydraulic fracturing.

4- Investigating the leakage of methane from the deep subsurface into shallow aquifers through overburden sediments.

5- Estimating the contamination arrival time, flow rate and concentration in shallow aquifers under varying scenarios.

6- Determining and ranking the most important parameters and processes influencing the upward migration of fluids.

7- Determining the critical combination of key parameters leading to the highest degree of groundwater contamination.

\subsection{Dissertation Overview}

The remainder of this dissertation is organized as follows.

Chapter 2 introduces the development of relevant conceptual models using a hydraulic fracturing FEP database. First, the FEP database (Tatomir et al., 2015; Wiener et al., 2015) is attached to the six focused modelling scenarios, previously described (Tatomir et al., 2016). Next, the database is ranked to identify the relevance of each factor in the FEP list per scenario. The ranked FEPs list is used to determine the critical combinations of factors (e.g. hydrogeology and operational factors) leading to the highest environmental risks. Finally, the ranked list served as the basis for developing conceptual models to study methane, fracturing fluid and brine migration from the hydrocarbon formation towards shallow groundwater.

Chapter 3 focuses on the short-term flow of fracturing fluid and brine from the gas formation to the aquifer along a conductive fault. The sensitivity analysis studies the influence of 
fracturing injection pressure, fault properties, overburden properties and salinity on the vertical extend of fluids. Additional analysis for inclined faults, as well as cases with the fault not extending to the aquifer, are conducted. Furthermore, the effect of domain boundary conditions on the upward flow of fluids are examined.

Chapter 4 focuses on fracturing fluid and brine migration along an abandoned well using the geological data of the North German Basin. The model simulates the influence of frac hits, i.e. interaction between hydraulic fractures and wells, on abandoned oil and gas wells. The numerical model studies fluid migration during various stages of a hydraulic fracturing well, namely fracturing fluid injection, shut-in, flowback, production and post-production periods. The sensitivity analysis examines the influence of fracturing fluid volume, hydrocarbon formation properties, overburden properties, salinity, abandoned well properties and its distance to the operation.

Chapter 5 focuses on the development of a nonlinear autoregressive (NAR) neural network to predict fracturing fluid flow rate to shallow groundwater in the presence of an abandoned well. The developed NAR model is trained using the Levenberg-Marquardt (LM) and Bayesian Regularization (BR) algorithms. The data obtained in chapter 4 are used for training and testing the NAR-LM and NAR-BR models.

Chapter 6 focuses on the transport of methane from a leaky natural gas well into a shallow aquifer at the basin scale. A two-dimensional, two-phase, two-component, generic model was used to investigate the migration of methane and brine through overburden rocks. The sensitivity analysis explores the influence of Brooks-Corey parameters and residual water saturation of overburden sediments, gas leakage rates and periods, tilted geometries and barrier units (i.e. clay layers) on the migration of methane and brine to shallow groundwater.

Chapter 7 summaries the findings, provides conclusions with respect to the focus of the dissertation, and gives future research recommendations. 


\section{Chapter 2}

\section{Conceptual model development using a generic features, events and processes (FEP) database}

This chapter is based on the following paper:

Conceptual model development using a generic Features, Events, and Processes (FEP) database for assessing the potential impact of hydraulic fracturing on groundwater aquifers

Alexandru Tatomir ${ }^{* 1}$, Christopher McDermott ${ }^{2}$, Jacob Bensabat ${ }^{3}$, Holger Class ${ }^{4}$, Katriona Edlmann ${ }^{2}$, Reza Taherdangkoo ${ }^{1}$, and Martin Sauter ${ }^{1}$

${ }^{*}$ Corresponding author

${ }^{1}$ Department of Applied Geology, University of Goettingen, Göttingen, 37077, Germany

${ }^{2}$ School of Geoscience, University of Edinburgh, Edinburgh, UK

${ }^{3}$ Environmental \& Water Resources Engineering (EWRE), Haifa, Israel

${ }^{4}$ Department of Hydromechanics and Modelling of Hydrosystems, University of Stuttgart, Stuttgart, 70569, Germany

Citation: Tatomir, A., McDermott, C., Bensabat, J., Class, H., Edlmann, K., Taherdangkoo, R. and Sauter, M., 2018. Conceptual model development using a generic Features, Events, and Processes (FEP) database for assessing the potential impact of hydraulic fracturing on groundwater aquifers. Advances in Geosciences, 45, pp.185-192. 


\begin{abstract}
Hydraulic fracturing for natural gas extraction from unconventional resources has not only influenced the global energy landscape, but also raised concerns over its potential environmental impacts. The concept of "features, events and processes" (FEP) refers to identifying and selecting the most relevant factors for safety assessment studies. In the context of hydraulic fracturing, we constructed a comprehensive FEP database and applied it to six key focused scenarios defined under the scope of FracRisk project. The FEP database is then ranked to show the relevance of each item in the FEP list per scenario. The main goal of this chapter is to illustrate the FEP database applicability to develop conceptual models for upward migration of fracturing fluid, brine and methane from a shale gas reservoir towards shallow groundwater at the field and regional scales.
\end{abstract}




\subsection{Introduction}

The application of new engineering techniques such as directional drilling and multi-stage hydraulic fracturing has opened up access to unconventional reservoirs, but the thought of injecting large quantities of pressurized chemical-laced water into underground has raised public concerns over its potential impacts on human health, drinking water, air quality and landscape. Initiatives aimed at understanding, preventing and mitigating the potential environmental impacts and risks of unconventional gas development were formulated for instance by the European Commission (e.g. Horizon 2020 program LCE-16-2014).

One of the public concerns is contamination of shallow groundwater via fracturing fluid and fugitive hydrocarbon gases (King, 2012; Kissinger et al., 2013; Lange et al., 2013; Vengosh et al., 2014). Fracturing and formation fluids may migrate upward if hydraulically induced fractures intercept the vicinity of fractured systems, faults, and abandoned wells (Brownlow et al., 2016; Taherdangkoo et al., 2017). The main scientific challenges are related to understanding the complex thermo-hydro-mechanical-chemical impacts of fracturing process and accounting for the wide range of heterogeneities occurring at various scales (pore-to fieldscale) in the geological formations. For addressing such complexities, within the FracRisk project, the main environmental risks were subdivided into six key focused conceptual scenarios, defined as S1 to S6 (Figure 2-1) and described in chapter 1.

Most commonly, during the exploration stage the focus is on determining the characteristics of the hydrocarbon bearing formation ( $\mathrm{HCBF})$, while less effort is dedicated for characterizing the overburden layers. This leads to a high degree of uncertainty in predicting flow and transport behavior of fluids and chemicals in the subsurface. The environmental risks of shale gas development can be evaluated by employing techniques developed for other engineered systems such as geological storage of carbon dioxide and radioactive waste repositories. In this sense, a structured qualitative approach for identifying and analyzing the key factors and processes relevant for environmental impact assessment is the features, events and processes (FEP) approach (Ayash et al., 2009; Lewicki et al., 2007; Paulley et al., 2011; Savage et al., 2004; Walke et al., 2011; Yavuz et al., 2009). The FEP describes the identification and selection of the relevant elements for hydraulic fracturing safety: characterization of the main geological features, definition of key events and understanding of main processes occurring during shale gas development. 
The objective of this work is to show the process of developing conceptual models for assessing the potential impacts on groundwater aquifers based on the FEP database. First, we describe the FEP database and the ranking of FEPs combined with the hydro-geo-mechanical-chemical facies and the source-pathway-receptor approaches. Second, we develop a conceptual model addressing the regional scale flow and transport of methane. This conceptual model is in the scope of scenario S5 (Figure 2-1) and is provided as an example. The value ranges of studied parameters (e.g. porosity, permeability, and formation depth) and applied boundary conditions are determined from data of seven shale gas basins in Europe (Baltic, Paris, North West German, Lublin, Bowland, Carpathian-Balkan, Pannonian-Transylvanian) and a chemical database (Tatomir et al., 2016).

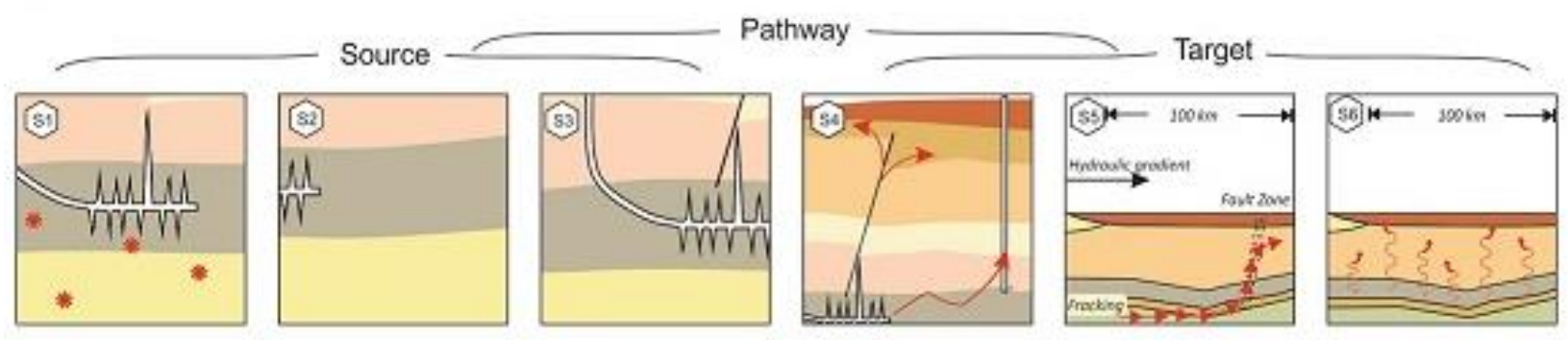

Figure 2-1. The six focused scenarios for the combined evaluation of FEPs. S1: hydraulic fracturing processes; S2: fluid injection, fluid migration and micro-seismics; S3: Source characterization, produced fluid and remaining fluid; S4: short-term flow and transport through fault zones and abandoned wells; S5: long-term regional transport of fluids; S6: long-term diffusive transport through overburden layers and aquifers (source: http://www.fracrisk.eu, Christopher Mcdermott).

\subsection{Features, events and processes analysis}

\subsubsection{General concepts}

"Features" represent the characteristics of a static system (e.g. porosity, permeability, and overburden thickness). "Events" refer to changes in the system or its environment because of fracturing (e.g. drilling of a horizontal borehole, casing emplacement, and cementation) or due to natural causes (e.g. earthquakes, and soil erosion). "Processes" describe the way the system attributes and conditions evolve with time. Processes are associated with the dynamic interactions between the features (e.g. mineral dissolution, advective transport, and buoyancy driven flow). To a certain extent, there is no clear delineation and overlapping between events 
and processes, and sometimes even features (e.g. hydrodynamic dispersion can be regarded as an attribute of the porous system, or as the spreading of a solute during its transport). The source, pathway, and receptor (SPR) conceptual approach is often used in hydrogeology as a basis for environmental impact assessment. The same approach is applied here, where the source compartment is the gas formation, the pathway is represented by faults, fracture networks and abandoned wells, and the receptor is the shallow aquifer.

According to the hydro-geo-chemical-mechanical (HGCM) facies concept described by McDermott et al. (2006), the subsurface is composed of a number of facies subdivided according to their mechanical and hydrogeological properties. The facies do not necessarily correspond to the geology but rather to their functional characteristics (e.g. aquifer, pathway, and rock seal). Therefore, mathematical models can be parametrized based on the HGCM facies concept. The advantage of applying HGCM facies approach is that it allows comparing different scenarios and locations in terms of their suitability to shale gas development. This provides a holistic framework for assessing the environmental impacts, which is essential for the development of scientific recommendations and legislative inputs.

A number of sub-scenarios are then constructed based on the FEP list (Tatomir et al., 2016). Each sub-scenario contains model assumptions, mathematical/numerical models, definition of boundary conditions and initial conditions, definition of input variables and the value ranges to be scanned in the sensitivity analysis and parameters of investigation (e.g. breakthrough curves, pressure and concentration). The criteria for identifying the fundamental differences between each scenario include geomechanics of fracture development, hydro-mechanics and geo-seismicity, driving forces (e.g. gravity, capillarity and vertical pressure gradient), time scales (e.g. fracturing process and fluids upward migration), spatial scale (near-field and farfield) and fluids (i.e. fracturing and formations fluids).

\subsubsection{FEP database construction}

The identification of FEPs and selection of the most relevant components for each focused scenario is an essential step. The starting point for developing the FEP database was the accessible database developed by Quintessa for $\mathrm{CO}_{2}$ storage systems (Savage et al., 2004; Walke et al., 2011). The list of features, events and processes was analyzed and discussed among the FracRisk consortium members and international partners during a two-day 
meeting. The FEPs list was modified to address the potential impacts on drinking water resources and to assess the level of risks associated with each focused scenario. The FEP list was then provided to the consortium members and the risk combinations were used to direct modelling and model development, and to access monitoring options to reduce uncertainty. The workshop was facilitated in the form of hazard identification.

The generic database of FEPs per scenario (Tatomir et al., 2015) refers to all the phenomena that may occur within shale gas development or that may impact upon it. The FEPs are used to assist in the identification of critical combinations of subsurface geology, operational practice of hydraulic fracturing and associated hazard and risk assessment for the natural and human environment. Note that the generic FEPs database is not specific to any particular fracturing operation or location. Figure 2-2 represents the main FEP elements for the shale gas development.

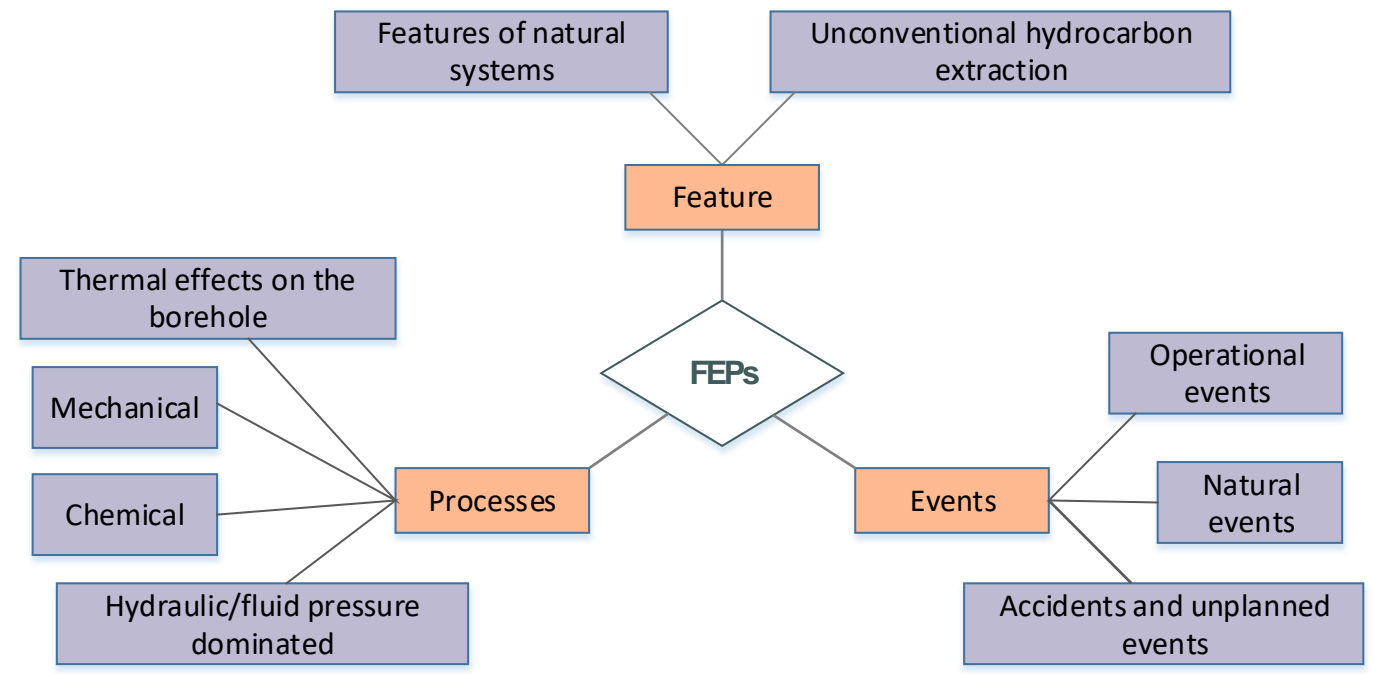

Figure 2-2. The main FEPs categories in environmental impact assessment of hydraulic fracturing for shale gas extraction.

\subsubsection{FEPs ranking}

There are several ways to assign the most relevant combination of FEPs to each scenario, such as (a) conducting field tests for assessing the importance of each item in the FEPs list, (b) collecting data from fracturing sites and (c) using the wisdom of a crowd. Options (a) and (b) are tedious to perform due to the high costs and technical challenges of running tests in deep geological formations and lack of data, respectively. Lavrakas (2008) defined ranking as a question response format used when a researcher is interested in establishing some type of 
priority among a set of objects. In this work, option (c) was chosen using the knowledge and experience of the FracRisk consortium members therefore the level of importance of each item in the FEP list for every scenario was ranked by all members of the project. The five-point Likert scale was used (Miller et al., 2007) to allow each participant to assign an importance value between 1 to 5 to each item representing (1) the least critical, (2) slightly critical, (3) critical, (4) considerably critical, and (5) the most critical. This response scale is applied because it is easier to describe five quality levels. For larger scales (e.g. seven points), the response task may become too specific and might cause confusion and non-uniformity between the respondents. Furthermore, being a comprehensive survey with three appraisal tables, using a larger scale can be demanding and experts may resort to round up their answers (Maitland, 2009).

The FEP appraisal tables (Wiener et al., 2015) were gathered from the project participants, with no restriction on the number of contributors from the same group. The appraisal tables were given the same weight. However, a sensitivity analysis can be conducted to determine the importance of individual FEPs with respect to the main goal of the focused scenario. The average value of importance for each item in the FEP list per scenario was then calculated. The ranking of each scenario is performed independently; thus, there is no link between the ranking processes of different scenarios. The highest ranked features, events, and processes are presented in Table 2-1, Table 2-2, and Table 2-3, respectively.

\subsection{Conceptual model development}

Conceptual models are simplified representations of hydrogeological systems, or sub-systems used to describe the main physical features and principal processes (Helmig, 1997). They represent the basis of all simulation models. The degree of abstractization and idealization of conceptual models is chosen such that there is a balance between the system complexity and computational costs. The essential system properties should remain clear in the conceptual model (Helmig, 1997). Herein, conceptual models provide information on fracture growth, pressure distribution, fluid flow, contamination transport, etc. Therefore, conceptual modelling requires an integrated approach based on hydrogeological, geochemical and geophysical data along with the information from fracturing operations. In the early stage of conceptual modelling, expert knowledge and access to the aforementioned information are limited, but as the development continues the knowledge and available data increase. 
Based on the identification of the key risk combinations, the possible parameter variations of the key input variables within the six focused scenarios can be characterized. The values are taken from the assessment of the different HGCM facies characteristics reported in the literature and within the seven different shale gas basins. This ensures that events and processes occurring at different sites can be compared and contrasted against a common frame of reference, providing the basis for the construction of a structured knowledge base and generic risk assessment.

The workflow process of developing conceptual models is illustrated in Figure 2-3. The generic FEP database can be applied in conjunction with two approaches, namely top-down and bottom-up (Walke et al., 2011). The top-down is a concern or hazard-based approach where scenarios are developed by considering a small number of high-level FEPs. The top-down approach can be derived from expert judgment of FEPs from specific cases, where only certain details about the system are known. This requires gradual addition of details into the model. The bottom-up approach considers combinations of all possible FEPs, which results in a large number of scenarios and combinations to be investigated.

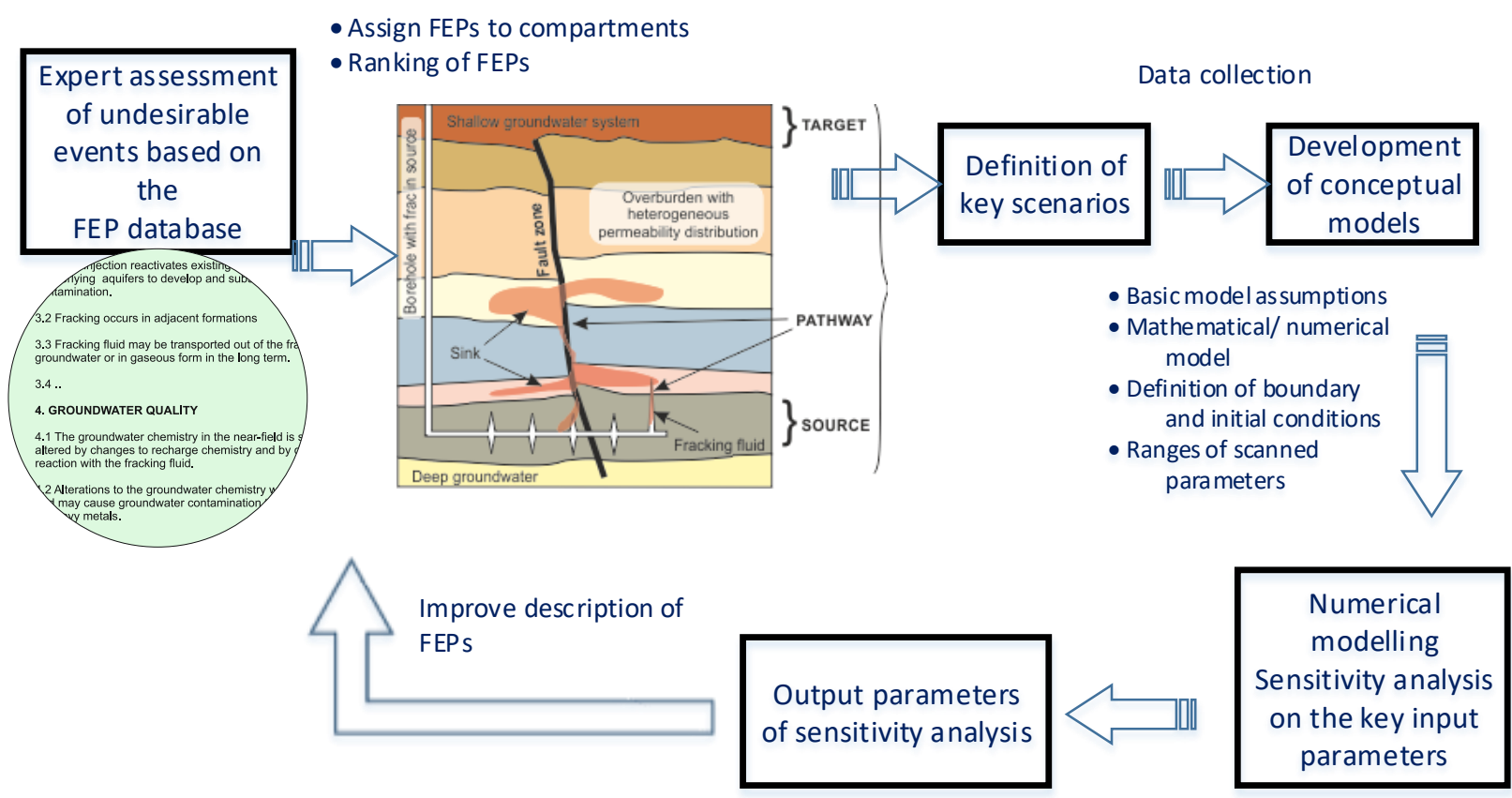

Figure 2-3. General workflow for the construction of the conceptual models. 


\subsection{Regional-scale stray gas migration conceptual model}

Evidences for methane contamination of drinking water associated with shale gas extraction in aquifers overlaying shale formations are available in the literature (e.g. Jackson et al., 2013; Osborn et al., 2011; Warner et al., 2012). Scenario S5 deals with the long-term transport of fluids in a conductive regional-scale aquifer (with the spatial extension ranging between 10 and 100 $\mathrm{km})$. The highest ranked events for this scenario are the insufficient site characterization, out of zone pumping, and cap rock failure (Wiener et al., 2015). These lead to the creation of pathways into a highly conductive aquifer in the overburden. The dominating processes are buoyancy and regional flow (Sauter et al., 2012). While it is difficult to setup a generic study for such a case, we focused exemplarily on an inclined aquifer, into which methane leaks and further spreads. Note that for this case, the assumptions made with respect to the setup of the simulations are rather conservative and do not reflect conditions that should normally be expected under real operating conditions.

Figure 2-4 represents the conceptual model for the failure scenario and gives an idea of how the geological environment, into which this scenario is embedded, could look like. According to the schematic illustration, it is seen that an inclined conductive aquifer can be modelled as a rectangular domain with an influx of methane assigned as a flux boundary condition.

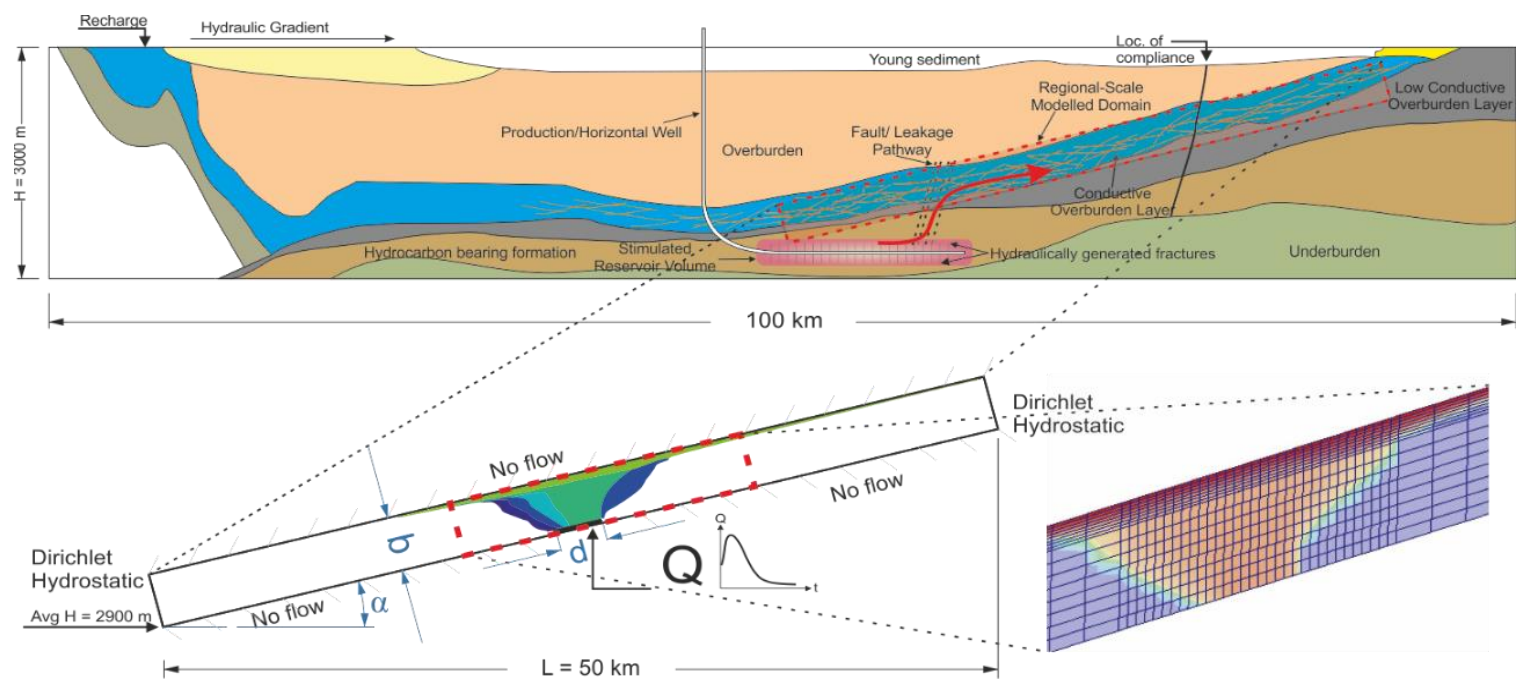

Figure 2-4. Regional scale conceptual model for migration of methane and fracturing fluids. 


\subsection{Conclusions}

We presented a FEP database for assessing the environmental risks of hydraulic fracturing on groundwater aquifers. We then demonstrated a procedure for developing conceptual models, which allows testing of various risk scenarios based on the critical combinations of FEPs. At the same time, the approach aimed at identifying the key processes that may affect the transport and fate behavior of contaminants in the subsurface. The FEP approach is flexible allowing to be applied generically or to a particular system (e.g. a specific site), using either a bottom-up or top-down approach. We finally presented a scenario dealing with the long-term response of geological setting to hydraulic fracturing at the regional scale. The outputs of FEP analysis help to channel further characterization and modelling efforts. One advantage of the FEP approach is that it facilitates the dialogue among the consortium partners and/or different stakeholders and leads to the identification of the key risks and uncertainties. The FEP approach helps filling the gaps in understanding of the relevant and important features and processes by employing a common language in a multidisciplinary consortium. Furthermore, it builds confidence that no relevant component is omitted in the risk assessment procedure.

Table 2-1. The highest ranked features for the six focused scenarios.

\begin{tabular}{|c|c|c|c|c|c|c|}
\hline List of Features & S1 & S2 & S3 & S4 & S5 & S6 \\
\hline \multicolumn{7}{|l|}{ A. Features of the Natural System } \\
\hline \multicolumn{7}{|l|}{ 1. Hydrogeology } \\
\hline \multicolumn{7}{|l|}{ 1.1. Hydrocarbon bearing formation (Source) } \\
\hline Rock / Petrophysical properties of the hydrocarbon bearing formation & 4 & 4 & & & & \\
\hline Lithology & 4 & 4 & 4 & 4 & & 4 \\
\hline Intrinsic permeability & 4 & 4 & & 4 & 4 & \\
\hline Relative permeability & & 4 & 4 & 5 & 4 & \\
\hline Entry pressure & & 4 & 4 & 4 & & \\
\hline Stress and Mechanical properties & 5 & & & & & \\
\hline Heterogeneity of the hydrocarbon bearing formation & 4 & & & & & \\
\hline Fractures and faults within the hydrocarbon bearing formation & 4 & 4 & 4 & 5 & 4 & \\
\hline Porosity of the fracture & & & & 4 & & \\
\hline Intrinsic permeability of the fracture & 4 & 4 & & 4 & & \\
\hline Relative Permeability of the fractures & & 4 & & 4 & 4 & \\
\hline Fracture geometry & & 4 & & 4 & & \\
\hline \multicolumn{7}{|l|}{ 1.2. Fluids } \\
\hline Hydrocarbons & & & 4 & & & \\
\hline Natural formation water & & & 4 & & & \\
\hline Production fluids & & & 4 & & & \\
\hline Pore fluid composition within the fracturing reservoir & & & 4 & & & \\
\hline
\end{tabular}




\begin{tabular}{|c|c|c|c|c|c|c|}
\hline Reservoir fluids & & & 4 & & & \\
\hline Other fluids & & & 4 & & & \\
\hline \multicolumn{7}{|l|}{ 1.3. Overburden } \\
\hline Porosity & & & & 4 & & \\
\hline Fractures and faults within the overburden & & 4 & & & & \\
\hline Relative Permeability of the fractures & & & & 4 & & \\
\hline Fracture geometry & & 4 & & 4 & & \\
\hline \multirow{2}{*}{\multicolumn{7}{|c|}{ Undetected features within the overburden }} \\
\hline & & & & & & 2. Near surface environment (Receptors) \\
\hline \multicolumn{7}{|l|}{ 2.1. Terrestrial environment } \\
\hline Near-surface aquifers and surface water bodies & & & & & 4 & 4 \\
\hline \multicolumn{7}{|l|}{ B. Unconventional Hydrocarbon Extraction } \\
\hline \multicolumn{7}{|l|}{ 1. Hydro-fracturing fluid } \\
\hline 1.1. Hydraulic injection fluid properties & & & 4 & & & \\
\hline \multicolumn{7}{|l|}{ 1.2. Physical properties of injection fluid } \\
\hline Injection fluid additives & & & 4 & & & \\
\hline \multicolumn{7}{|l|}{ 3. Site operation } \\
\hline \multicolumn{7}{|l|}{ 3.1. Drilling and completion } \\
\hline Horizontal wells & 4 & 4 & & & & \\
\hline \multicolumn{7}{|l|}{ 4. Site decommissioning } \\
\hline Abandoned wells & & & & 4 & & \\
\hline
\end{tabular}

Table 2-2. The highest ranked events for the six focused scenarios.

\begin{tabular}{|c|c|c|c|c|c|c|}
\hline List of Events & S1 & S2 & S3 & S4 & S5 & S6 \\
\hline \multicolumn{7}{|l|}{ 1. Operational Events } \\
\hline Hydraulic fracturing & 5 & 4 & 4 & 4 & & \\
\hline Out of zone / beyond pumping & & & & 4 & 4 & 4 \\
\hline Production & & 4 & 4 & 4 & & \\
\hline Seal failure & & 4 & & & 4 & \\
\hline \multicolumn{7}{|l|}{ 2. Natural events } \\
\hline Earthquakes & 4 & & & & & \\
\hline Cap rock failure & & 4 & & 4 & & \\
\hline \multicolumn{7}{|l|}{ 3. Accidents and unplanned events } \\
\hline Overpressuring & 4 & 4 & 4 & 4 & & \\
\hline Poor site characterization & & 4 & 4 & 4 & 4 & \\
\hline Incorrect chemical mix released into fracuring fluid & & & 4 & & & \\
\hline Cementation poorly undertaken (spaces left) & & 4 & 4 & 4 & & \\
\hline
\end{tabular}

Table 2-3. The highest ranked processes for the six focused scenarios.

\begin{tabular}{|c|c|c|c|c|c|c|}
\hline List of Processes & S1 & S2 & S3 & S4 & S5 & S6 \\
\hline \multicolumn{7}{|l|}{ 2. Hydraulics / Fluid Pressure Dominated } \\
\hline $\begin{array}{l}\text { Fluid pressure exceeds rock fracturing pressures generating new } \\
\text { fractures }\end{array}$ & 4 & 4 & & 4 & & \\
\hline Fluid exceeds fault sealing pressures & 4 & 4 & & 4 & & \\
\hline Fluid pressure exceeds stability of part of the plant construction. & 4 & 4 & & & & \\
\hline Displacement of surrounding formation fluids & & 4 & & 4 & 4 & \\
\hline
\end{tabular}




\begin{tabular}{|c|c|c|c|c|c|c|}
\hline Buoyancy-driven flow & & 4 & & 4 & 4 & 4 \\
\hline Advection and co-migration of other gas & & & 4 & & & \\
\hline Water mediated transport & & & 4 & & 4 & \\
\hline Advection & & & 4 & 4 & 4 & 4 \\
\hline Dispersion & & & & 4 & 4 & 4 \\
\hline Diffusion & & & 4 & & & 4 \\
\hline $\begin{array}{l}\text { Hydraulic and production fluids and the associated contaminants } \\
\text { release processes }\end{array}$ & & & 4 & 4 & 4 & 4 \\
\hline \multicolumn{7}{|l|}{ 3. Chemical } \\
\hline Corrosive mixture attacks plant & & & 4 & & & \\
\hline Corrosive mixture attacks geology & & & 4 & & & \\
\hline Sorption and desorption & & & 4 & & & \\
\hline Mineral dissolution & & & 4 & 4 & & \\
\hline Heavy metal release & & & 4 & 4 & & \\
\hline \multicolumn{7}{|l|}{ 4. Mechanical } \\
\hline Soil and rock deformation around boreholes & 4 & 4 & & 4 & & \\
\hline Propagation of fractures beyond the target zone & 4 & 4 & 4 & 5 & & \\
\hline Fluid exceeds fault sealing pressures & 4 & 4 & & 5 & & \\
\hline Fault valving & 4 & 4 & & 4 & & \\
\hline Micro-cracking in the casing cements & & 4 & & 4 & & \\
\hline
\end{tabular}




\section{Chapter 3}

\section{Fracturing fluid migration along a fault zone}

This chapter is based on the following paper:

Numerical investigations of upward migration of fracking fluid along a fault zone during and after stimulation

Reza Taherdangkoo*, Alexandru Tatomir, Robert Taylor, Martin Sauter

*Corresponding author

Department of Applied Geology, Geosciences Center, University of Göttingen, Goldschmidtstr. 3, D-37077 Göttingen, Germany

Citation: Taherdangkoo, R., Tatomir, A., Taylor, R. and Sauter, M., 2017. Numerical investigations of upward migration of fracking fluid along a fault zone during and after stimulation. Energy Procedia, 125, pp.126-135. 


\begin{abstract}
This chapter investigates the potential migration of fracturing fluid to shallow groundwater as a result of hydraulic fracturing in the vicinity of a conductive fault zone. We develop a 2D single-phase multispecies numerical model to study fracturing fluid flow along a permeable fault connecting the shale formation to the aquifer. A series of sensitivity analysis is considered to understand the influence of key parameters and mechanisms on the upward migration of fluids. The results show that fracturing fluid may reach the aquifer under specific conditions such as the presence of a highly permeable fault. Modelling demonstrates fault zone properties and fracturing pressure strongly dominate vertical fluid flow towards the aquifer. It is manifested hydraulic fracturing should not be conducted in proximity to pre-existing permeable pathways.
\end{abstract}




\subsection{Introduction}

Hydrocarbon production from unconventional resources (e.g., shale and tight gas reservoirs) has gained considerable attention from the oil and gas industry to cover the global increase in energy demand. Shale and tight gas reservoirs display relatively low matrix permeabilities, thus making it difficult and energy inefficient to produce the gas using standard procedures (King, 2012; U.S. EPA, 2015). Hydraulic fracturing improves access to the larger area of the reservoir by creating artificial fracture networks, increasing reservoir permeability, and the contact areas across which fluids flow from the matrix to fractures. The generally applied method involves a stage-based, high-pressurized fracturing fluid injection into the formation for a short period of time to crack the rock (Kissinger et al., 2013; Lange et al., 2013; Li et al., 2015).

Hydraulic fracturing is a relatively new technology and its potential impacts on water resources has caused concern among the public. Recently, the EU funded "FracRisk" project within the Horizon 2020 framework program has initiated efforts to investigate the probability of in-situ contamination possibilities of the operation. A features, events, and processes (FEP) database was constructed and provided in a discussion platform to identify, rank, and assess the most critical failure scenarios in fracturing operations (Tatomir et al., 2016, 2015; Wiener et al., 2015). This research method dissects the topic by individually researching six possible scenarios in detail, in which the identification and connections of sources (e.g. gas reservoirs), pathways (e.g. faults), and targets/receptors (e.g. neighboring aquifers) are evaluated (Gläser et al., 2016; Wiener et al., 2015).

Currently, a limited number of numerical modelling based research exploring the leakage through fault zones are available (Birdsell et al., 2015a; Gassiat et al., 2013; Kissinger et al., 2013; Myers, 2012a; Pfunt et al., 2016; Reagan et al., 2015). Myers (2012) produced a Marcellus shale based model to simulate fracturing fluid flow from the shale to the overlying aquifer. He concluded that the presence of a permeable pathway decreases the arrival time of fracturing fluid to the aquifer. Kissinger et al. (2013) presented three different scenarios addressing the short- and long-term movement of fracturing fluid, brine, and methane into the overburden applying both local and regional scales. Gassiat et al. (2013) utilized a simplified generic twodimensional model to assess the long-term impact of fracturing fluid migration into geological layers resulting from fracturing within an overpressurized shale reservoir. Birdsell et al. (2015) 
presented a two-dimensional model that accounted for various phases of the operation. They concluded that the combined influence of well production and capillary imbibition significantly reduce the risk of aquifer contamination. Pfunt et al. (2016) applied a twodimensional model to simulate the upward migration of fracturing fluid into the aquifer in the North German Basin. They deduced that fracturing fluid could not reach the aquifer regardless of the presence of a permeable pathway.

We constructed a generic, finite element method (FEM) based numerical model to simulate short-term fracturing fluid migration into overburden layers with the presence of a fault zone extending from the shale to the shallow aquifer. The simulation replicates two fracturing stages namely, injection and shut-in periods. A sensitivity analysis is conducted to examine the degree of influence of operational parameters and basin properties such as, injection pressure, pathway and overburden properties. We then investigate the effect of domain boundary conditions on the upward flow of fracturing fluid.

\subsection{Conceptual Model}

We build a generic model that could be applicable for further investigation of fluid leakage due to hydraulic fracturing, not to study a specific formation. To assess the potential worstcase scenario, a relatively simple model with a permeable continuous fault zone connecting the gas reservoir to the aquifer is considered. The reason for implementing a basic model is to improve the understanding of fracturing fluid migration mechanisms occurring within geologic layers. Note that this study does not describe fracturing processes relating to fracture propagation, dilatation, and fracture network formation; but rather applies assumptions of an already formed fracture network being present prior to injection. The model utilizes the assumption that fractured networks extend to the top of the gas reservoir. We simulate the typical injection and shut-in periods of the operation.

We built a two-dimensional geological conceptual model, Figure 3-1, comprised of three hydrostratigraphic units including: (1) aquifer, (2) overburden, and (3) the gas reservoir. A single fault zone extending from the reservoir to the aquifer is considered, which is a conservative assumption similar to those employed in previous studies (e.g. Gassiat et al., 2013; Kissinger et al., 2013; Myers, 2012). The presence of a connecting fault zone may not 
necessarily apply in nature that frequently, therefore, we also consider cases with a fault not reaching the aquifer (Edlmann and McDermott, 2016). Two failure scenarios are assumed including: (1) FS1; the presence of the HCBF; (2) FS2; the absence of the HCBF (Figure 3-1). With respect to the symmetry, the left edge of the model is assigned with a no-flow boundary. The top boundary of the domain is considered as the bottom of the aquifer. Instead of modelling the aquifer explicitly, we consider the aquifer as a constant pressure boundary at the top of the domain. We assume that any possible contamination that reaches the top boundary of the model enters the aquifer. To investigate the effect of different boundary conditions on the fluid migration, each scenario is divided into four sub-scenarios varying boundary conditions: (1) open (right: constant-head pressure boundary, bottom: pressure boundary); (2) closed (right: no-flow boundary, bottom: no-flow boundary); (3) semi 1 (right: constant-head pressure boundary, bottom: no-flow boundary); (4) semi ll (right: no-flow boundary, bottom: pressure boundary) Figure 3-1(b-c).

(b)
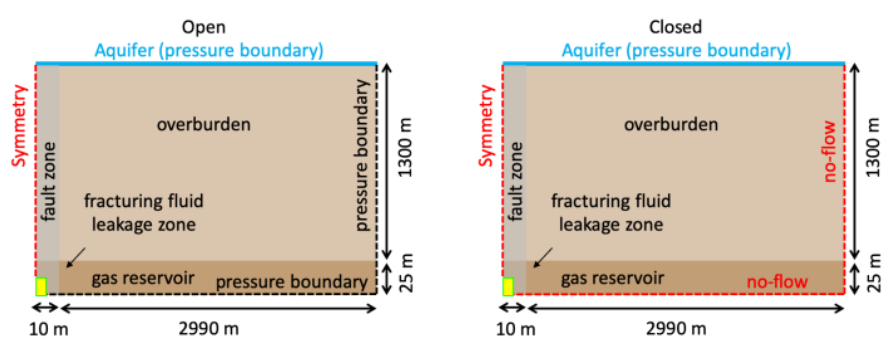

(a)
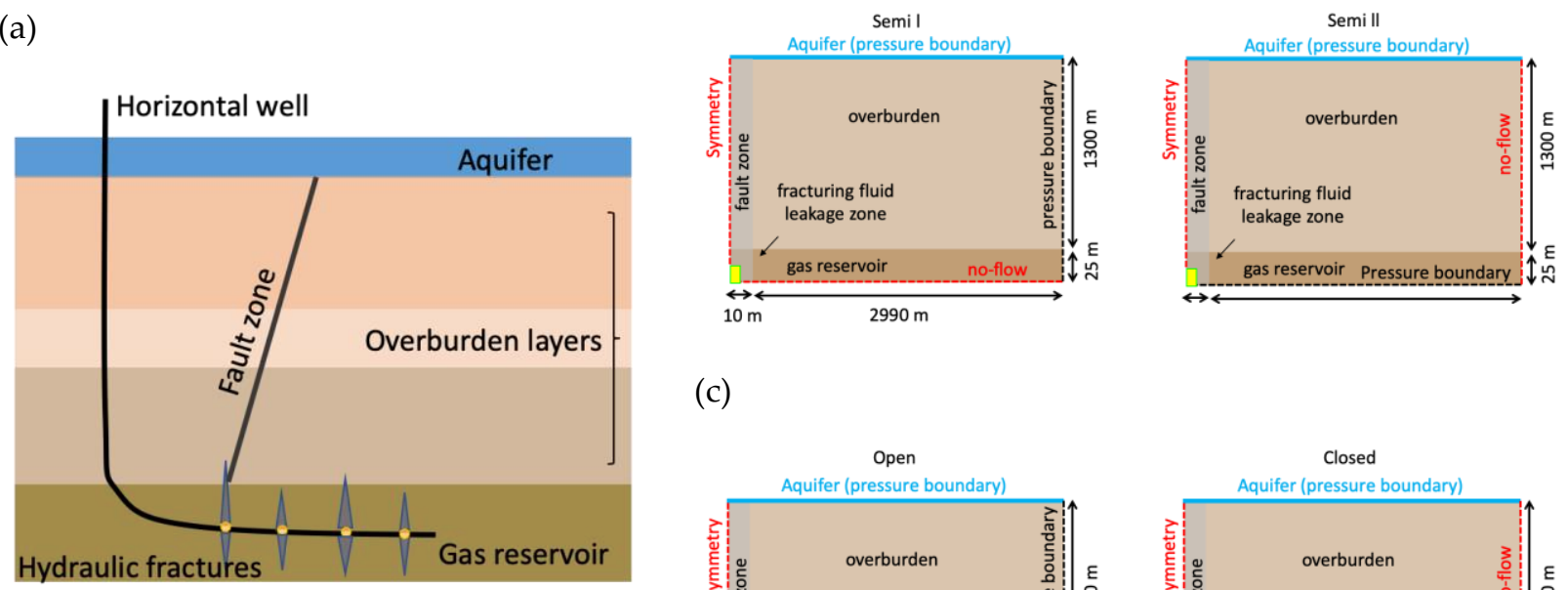

(c)
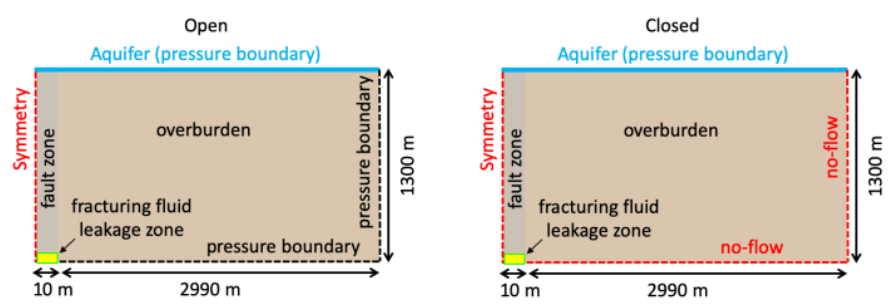

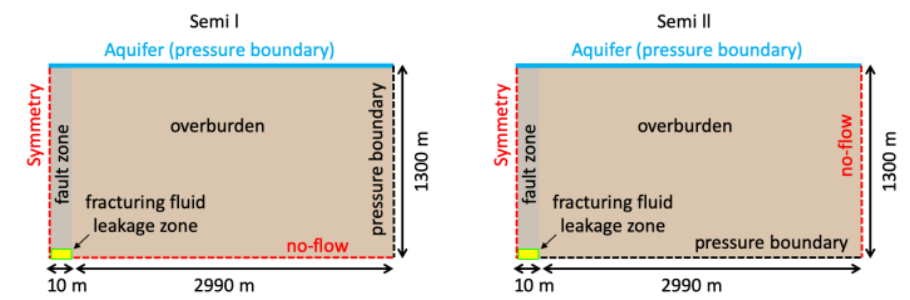

Figure 3-1. (a) A cross section schematic of a fault zone intersecting the stimulated reservoir volume $(\mathrm{SRV})$ in a hydrocarbon bearing formation and the conceptual representation of the source (HCBF) pathway (conductive fault) - receptor (aquifer). (b) FS1: with considering the HCBF and (c) FS2: without considering the HCBF.

The model domain has $1300 \mathrm{~m}$ depth and $3000 \mathrm{~m}$ width. All strata are assumed as homogeneous and anisotropic. Anisotropic conditions are assigned because horizontal permeability is often larger than vertical permeability by several orders of magnitude (Freeze and Cherry, 1979; Neuzil, 1994). The fault is considered as homogeneous and isotropic with a given permeability higher than the surrounding formations. The width of the fault is set to 10 m. Density and dynamic viscosity are calculated using Batzle \& Wang (1992) equations. An initial salinity of $100 \mathrm{~kg} / \mathrm{m}^{3}$ is set at the aquifer base and a moderate salinity gradient of 0.11 $\mathrm{kg} / \mathrm{m}^{3} / \mathrm{m}$ is assigned thorough the entire model (Gassiat et al., 2013; Pfunt et al., 2016). Density differences between fracturing fluid and brine lead to buoyancy forces. As high salinities are common in sedimentary basins, we investigate the effect of a wide range of salinities on fluid migration. An initial temperature of $12{ }^{\circ} \mathrm{C}$ is assumed at the aquifer base with an average temperature gradient of $0.03^{\circ} \mathrm{C} / \mathrm{m}$.

Table 3-1. Parameters used for base case models and sensitivity analysis.

\begin{tabular}{|l|c|c|l|}
\hline Parameters & Value & Variation & References \\
\hline Overburden thickness, $m$ & 1300 & $1100-2100$ & $\begin{array}{l}\text { (Arthur et al., 2008; Kissinger et al., 2013; US } \\
\text { EIA, 2011a) }\end{array}$ \\
\hline Fault width, $m$ & 10 & $5-20$ & $\begin{array}{l}\text { (Birdsell et al., 2015a; Gassiat et al., 2013; } \\
\text { Kissinger et al., 2013; Myers, 2012a; Pfunt et } \\
\text { al., 2016) }\end{array}$ \\
\hline $\begin{array}{l}\text { Overburden } \\
\text { permeability, } m^{2}\end{array}$ & $2 \times 10^{-16}$ & $\begin{array}{c}1 \times 10^{-18}- \\
1 \times 10^{-15}\end{array}$ & $\begin{array}{l}\text { (Gassiat et al., 2013; Gleeson et al., 2011; } \\
\text { Kissinger et al., 2013; Luijendijk and } \\
\text { Gleeson, 2015) }\end{array}$ \\
\hline $\begin{array}{l}\text { Overburden anisotropy } \\
\text { ratio }\end{array}$ & 100 & $1-1000$ & \begin{tabular}{l} 
(Freeze and Cherry, 1979; Neuzil, 1994) \\
\hline Fault permeability, $m^{2}$
\end{tabular} \\
\hline Fault porosity & 0.1 & $0.08-0.12$ & $\begin{array}{l}\text { (Kissinger et al., 2013; Reagan et al., 2015; } \\
\text { Saiers and Barth, 2012) }\end{array}$ \\
\hline
\end{tabular}




\begin{tabular}{|l|c|c|l|}
\hline Salt concentration, $\mathrm{kg} / \mathrm{m}^{3}$ & $\begin{array}{c}\text { Depth } \\
\text { dependent }\end{array}$ & 150 - 350 & (Ferguson et al., 2018; King, 2012) \\
\hline $\begin{array}{l}\text { Fracturing injection } \\
\text { pressure, } \mathrm{MPa}\end{array}$ & 30 & $20-45$ & $\begin{array}{l}\text { (Birdsell et al., 2015a; Gassiat et al., 2013; } \\
\text { Kissinger et al., 2013; Pfunt et al., 2016) }\end{array}$ \\
\hline
\end{tabular}

The fracturing operation is simulated by applying a constant pressure boundary at the edge of the fault zone. The fracturing injection pressure primarily depends on the petrophysical properties, depth, and initial lithological pressure of the gas formation (Ren et al., 2016; Yu and Aguilera, 2012; Zheltov, 1955). A sensitivity analysis of excess pressure values is performed to cover a wide range of injection pressures observed in the industry. During the injection period, the fracturing pressure is held constant for the 20-hour duration. Notably, the absence of a pressure reduction occurring between the borehole and the fault zone should be viewed as a conservative assumption. Considering a constant pressure at the fault zone to simulate the operation leads to an overestimation of the fracturing fluid concentration in the aquifer. For the base-case models, simulations of fracturing and shut-in periods are conducted in two consecutive runs. Total simulation time is 60 hours, of which the injection time is 20 hours (initial run), and the remaining 40 hours represents the shut-in period (second run). The output from the fracturing simulation is assigned as initial conditions for the second run. Since the purpose of this study is primarily to assess parameter influence on the upward migration of fracturing fluid, the sensitivity analysis was conducted only for the injection period. Therefore, by obtaining a "base case" reference model output from predefined mid-range values, individual parameter sweeps are conducted to identify the degree of numerical output alterations. The base case value of each parameter and its range of variation in the sensitivity analysis are presented in Table 3-1. The value of parameters used for all the simulations are shown in Table 3-2 (e.g. Gassiat et al., 2013; Kissinger et al., 2013; Sauter et al., 2012; US EIA, 2011a). 
Table 3-2. Parameters used in all of the simulations.

\begin{tabular}{|l|l|}
\hline Parameters & Value \\
\hline Domain length, $\mathrm{m}$ & 3000 \\
\hline Aquifer thickness, $\mathrm{m}$ & 100 \\
\hline Reservoir permeability, $\mathrm{m}^{2}$ & $1 \times 10^{-19}$ \\
\hline Aquifer pressure, $\mathrm{MPa}$ & 1 \\
\hline Overburden porosity & 0.03 \\
\hline Reservoir porosity & 0.03 \\
\hline Reservoir thickness, $\mathrm{m}$ & 25 \\
\hline Aquifer temperature, ${ }^{\circ} \mathrm{C}$ & 12 \\
\hline Reservoir overpressure gradient, $\mathrm{KPa} / \mathrm{m}$ & 13 \\
\hline Initial salinity at $200 \mathrm{~m}, \mathrm{~kg} / \mathrm{m}^{3}$ & 100 \\
\hline Fault anisotropy & 1 \\
\hline Geothermal gradient, ${ }^{\circ} \mathrm{C} / \mathrm{m}$ & 0.03 \\
\hline Reservoir anisotropy & 1000 \\
\hline Molecular diffusivity of solutes in pure fluid, $\mathrm{m}^{2} / \mathrm{s}$ & $1 \times 10^{-9}$ \\
\hline Fracturing fluid density, $\mathrm{kg} / \mathrm{m}^{3}$ & 1000 \\
\hline
\end{tabular}

\subsection{Mathematical and numerical model}

Fracturing fluid is a water-based fluid with fine sand grains and chemical additives (Li et al., 2015). The transport of fracturing fluid via gas migration is considered unlikely because after a certain transport distance gas and liquid phases are separated and continue individually (Schwartz, 2015). Therefore, the model assumes single-phase flow comprising two components. The model assumes fracturing fluid as a conservative tracer, i.e. concentration losses from sorption and degradation processes are ignored. The model does not account for geomechanical effects, such as dynamic changes in pore volume and permeability due to stress changes. The following set of equations describe the flow and solute transport in a porous media system (Bear, 2013; Helmig, 1997):

$$
\begin{aligned}
& u=-\boldsymbol{K} \frac{1}{\mu_{w}}\left(\nabla p_{w}+\rho_{w} \mathrm{~g}\right) \\
& \frac{\partial \phi \rho_{w}}{\partial t}-\nabla \cdot\left[K \frac{\rho_{w}}{\mu_{w}}\left(\nabla p_{w}-\rho_{w} \mathrm{~g}\right)\right]=0 \\
& \frac{\partial c_{i}}{\partial t}-\nabla \cdot\left(D_{p m}^{i} \nabla c_{i}\right)+u \cdot \nabla c_{i}-R_{i}=0
\end{aligned}
$$

where $u$ is the Darcy velocity or specific discharge vector (SI unit: $m / s$ ), $\boldsymbol{K}$ is the intrinsic permeability of the porous medium (SI unit: $m^{2}$ ); $\mu_{w}$ is the dynamic viscosity of water (SI unit: $P a . s$ ); $p_{w}$ is the fluid's pressure (SI unit: $\left.P a\right), \rho_{w}$ is the density (SI unit: $\mathrm{kg} / \mathrm{m}^{3}$ ); $g$ is the vector of gravitational acceleration; $c_{i}$ is the concentration of the chemical species $i$ (SI unit: $\mathrm{mol} / \mathrm{m}^{3}$ ); 
$D_{p m}^{i}$ is the diffusion coefficient of component $i$ (SI unit: $\mathrm{m}^{2} / \mathrm{s}$ ); $R_{i}$ is the reaction rate, which is set to zero. This is a conservative assumption that the fluid is not being absorbed by the porous media, nor interacts with it.

The mathematical model is implemented in COMSOL 5.2.a Multiphysics simulator, using the pre-existing Subsurface Flow Module by coupling the Darcy's Law interface with the Transport of Diluted Species in Porous Media interface. COMSOL Multiphysics is a finite element analysis (FEA) software package for various physics and engineering applications that discretizes the mathematical model by the finite element method.

\subsection{Results and discussion}

\subsubsection{Base-case model}

The modelling results of the two main failure scenarios (FS1, FS2), respectively the corresponding eight sub-scenarios (defined in section 3.2) are described and compared to better understand the influence of boundary conditions on the upward movement of fluids into overlying layers. The results are provided as fracturing fluid concentration breakthrough curves, concentration profiles along the fault zone, pressure profiles along the fault zone, and depth of $2 \%$ concentration threshold.

A very fine, unstructured, free-triangular, finite element mesh was generated for the discretization of the model domain. A sensitivity analysis with regard to the mesh size was conducted, identifying the optimum element size in which alterations to it produce minimal influences on the results. The applied mesh consisting of 105,368 elements is shown in Figure 3-2a. An example of the spatial distribution of the fracturing fluid concentration through the domain is illustrated in Figure 3-2b. For this case (FS2; sub-scenario: open), fracturing fluid migrated up the fault, and the $2 \%$ concentration threshold reached a depth of $330 \mathrm{~m}$ (respectively $970 \mathrm{~m}$ up the fault from the source location) following the shut-in period. This modelling scenario shows that a significant amount of fracturing fluid is migrated upward through the fault.

Figure 3-3 compares concentration breakthrough curves of the base cases of all eight subscenarios. It was observed that the vertical extent of fluids increased over the injection period, and maintained the level during the shut-in period. The plume spread more laterally during 
the shut-in period. The sub-scenarios "open" and "semi I" had lower concentrations arriving at the aquifer base when compared to sub-scenarios "closed" and "semi II". This applies to both main failure scenarios. This behaviour can be explained by the pressure distributions along the fault zones. The no-flow boundary conditions do not allow the fluid to leave the system and implicitly lead to a higher increase of fluid pressure in the entire domain compared to the pressure boundary condition, which allows fluxes outside of the domain. Therefore, as illustrated by Figure 3-4i and Figure 3-5i, the pressure gradients along the fault for subscenarios "closed" and "semi II" are higher than "open" and "semi I", leading to higher vertical flow, and subsequently larger fracturing fluid concentrations at the aquifer.

(a)

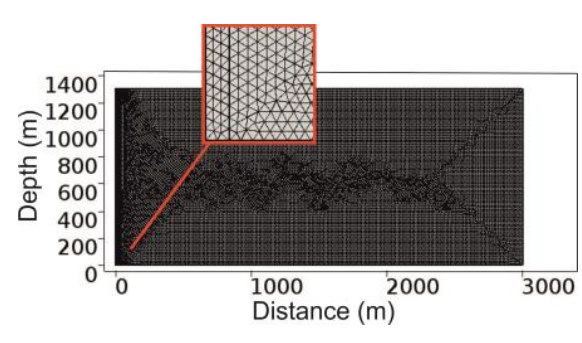

(b)

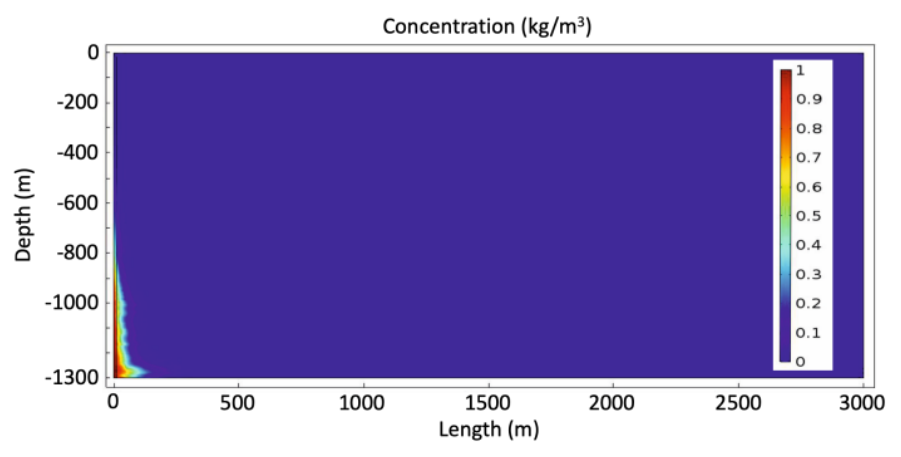

Figure 3-2. (a) Domain discretization for the numerical simulation and (b) fracturing fluid concentration profile at the end of shut-in period (FS1; sub-scenario: open).

Comparing the two failure scenarios, it was observed that for FS1 the fluid migrated further up the fault (shown by both sets of sub-scenarios). This is attributed to the overpressure within the reservoir. The highest concentration was monitored at the aquifer base $(1.2 \%$ of the initial concentration) in FS1, the "semi II" sub-scenario. In this scenario, 2\% level of concentration was reached to the height of $1236 \mathrm{~m}$. The lowest concentration in the aquifer was observed in FS2; sub-scenario "open". In this case, $0.16 \%$ of the initial concentration entered the aquifer and the height of $2 \%$ concentration threshold reached $970 \mathrm{~m}$.

When observing strictly the base case scenario for FS1 in sub-scenarios "closed" and "semi II", changing the bottom boundary from the constant-pressure magnitude to no-flow led to an increase of lateral spreading of the plume in the overburden, thus decreasing the concentration distribution in the aquifer. Moreover, for the base case scenario of FS2, both sub-scenarios "closed" and "semi II" led to the similar fluid concentration in the aquifer. Therefore, it is 
concluded that while the right side of the domain maintained constant-head pressure boundary, a change in the bottom boundary from the constant pressure to no-flow has negligible effect on the vertical extent of the plume and the respective concentration distribution at the aquifer base. From all of the investigated sub-scenarios, it was observed that changing the right domain boundary has a greater influence on the concentration at the aquifer base comparing to change of the bottom boundary condition.

(a)

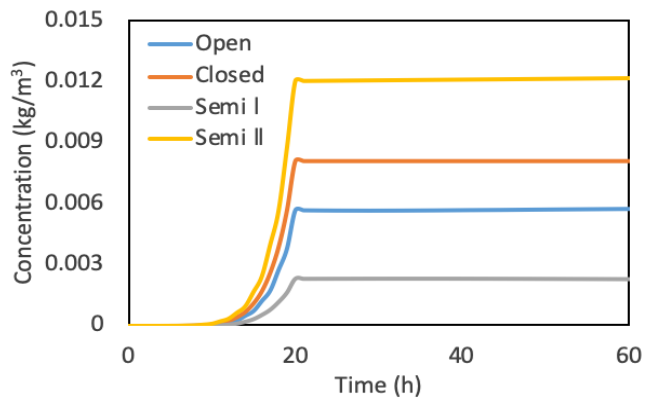

(b)

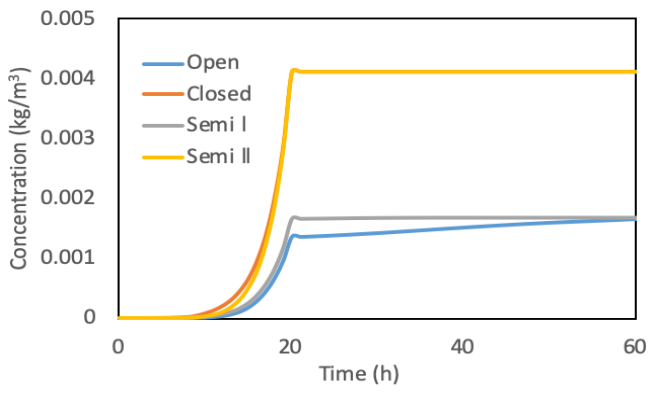

Figure 3-3. The concentration of fracturing fluid dissolved chemicals at the aquifer base throughout injection and shut-in periods, observed at the aquifer base. (a) FS1 and (b) FS2.

\subsubsection{Sensitivity analysis}

We performed a series of sensitivity analysis to understand the influence of operational parameters and reservoir physical properties. The studied parameters are fault permeability $\left(K_{f}\right)$, effective fault porosity $\left(\emptyset_{f}\right)$, fault width $\left(W_{f}\right)$, overburden thickness $\left(h_{o}\right)$, overburden permeability $\left(K_{o}\right)$, overburden anisotropy ratio $\left(A_{o}\right)$, fracturing injection pressure $\left(P_{\text {inj }}\right)$, and salinity $(S)$. We also considered cases where the fault zone does not extend to the aquifer, as well as instances where varying fault inclination are present. The concentration of fracturing fluid at the aquifer base at the end of the injection period is shown in Figure 3-4 and Figure 3-5. These figures demonstrate the sensitivity analysis results for the investigated parameters for all eight sub-scenarios. The model results showed fracturing fluid does not reach the aquifer base for inclined faults. In addition, scenarios without a continuous fault connecting the reservoir to the overlying aquifer resulted in a limited vertical migration of fluids. It is manifested the upward flow of fluids is strongly dependent on the vertical extend of the fault.

The results showed that a relatively small increase in the fault permeability led to a higher contamination distribution at the aquifer base. Fracturing fluid did not reach the aquifer base when the permeability of the fault was lower than $4 \times 10^{-11} \mathrm{~m}^{2}$, and the highest aquifer 
contamination occurred for the fault permeability higher than $7 \times 10^{-11} \mathrm{~m}^{2}$. For all subscenarios, an injection pressure higher than $30 \mathrm{MPa}$ resulted in the fracturing fluid arrival at the aquifer base. An excess in the injection pressure increases the upward migration of the fluid into the overburden. The increase of fault width increased the vertical flow of fracturing fluid towards the aquifer. The increase of overburden thickness decreases the likelihood of fracturing fluid arrival at the aquifer base. The increase of the overburden permeability and anisotropy increased the lateral spreading of fracturing fluid in the overburden, and therefore less concentration arrived at the aquifer. Furthermore, salinity gradients have the potential to exclusively influence the transport of fluids into overburden layers. The fluid propagation height was reduced with the increase of the salinity in the domain.

(a)

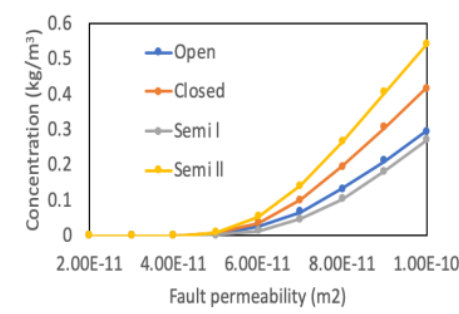

(d)

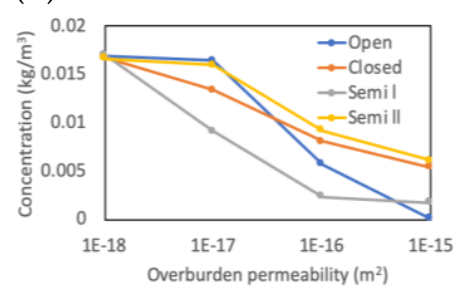

(g)

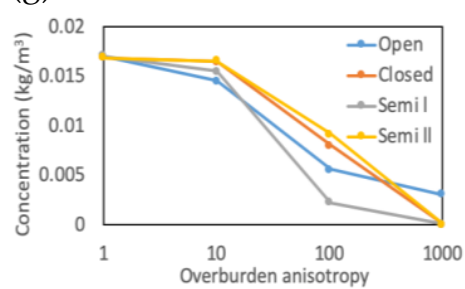

(b)

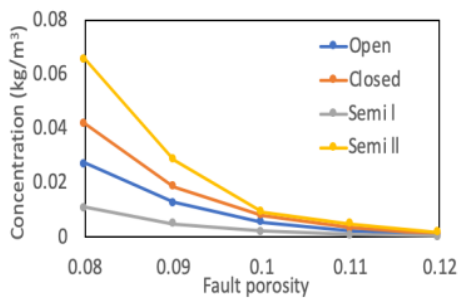

(e)

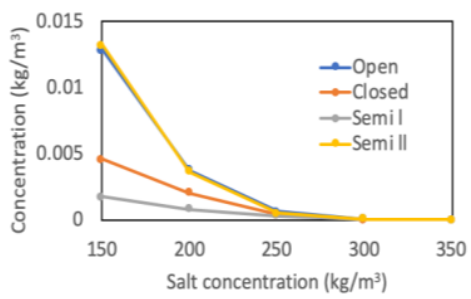

(h)

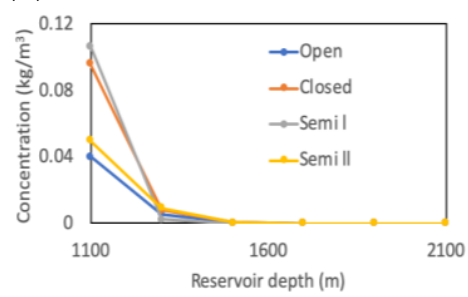

(c)

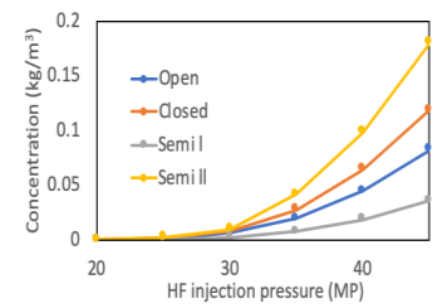

(f)

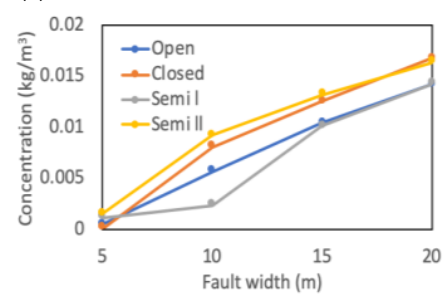

(i)

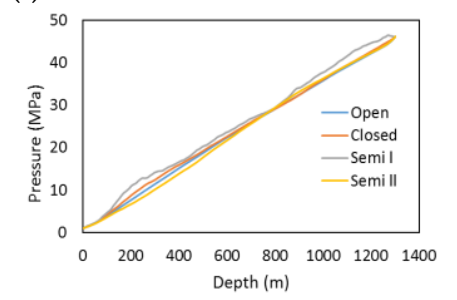

Figure 3-4. FS1 (model considering the presence of HCBF). (a-h) The concentration of fracturing fluid dissolved chemicals at the aquifer base at the end of the injection period for each investigated parameter range, and (i) pressure profile along the fault zone for four "base-case" sub-scenarios. 
(a)

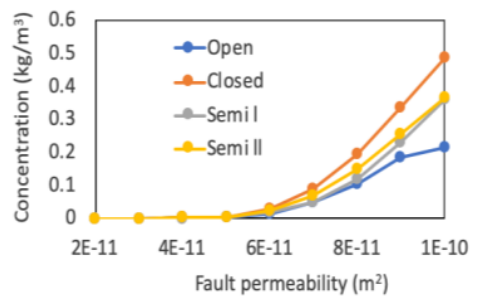

(d)

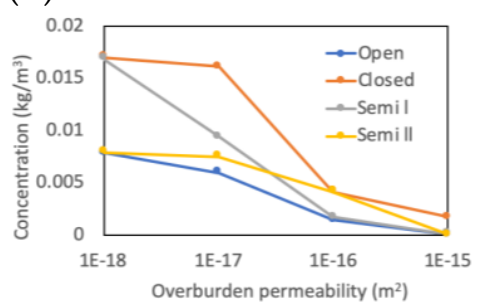

(g)

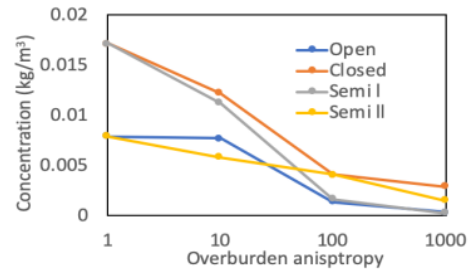

(b)

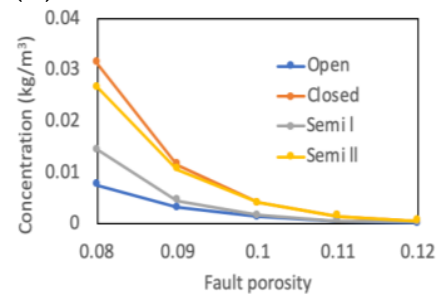

(e)

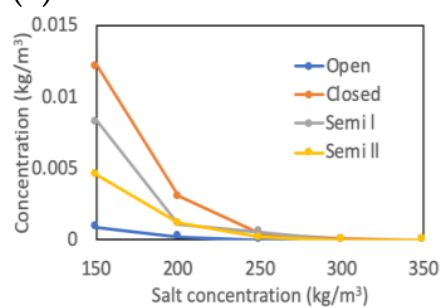

(h)

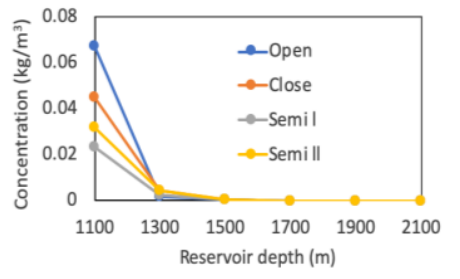

(c)

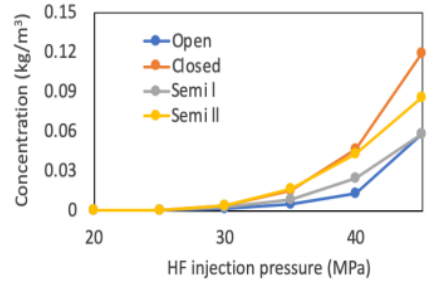

(f)

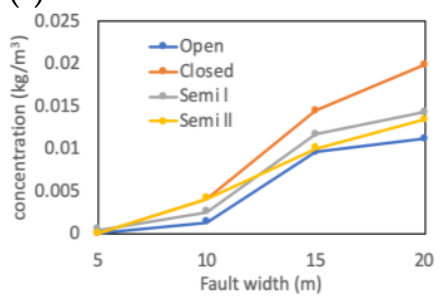

(i)

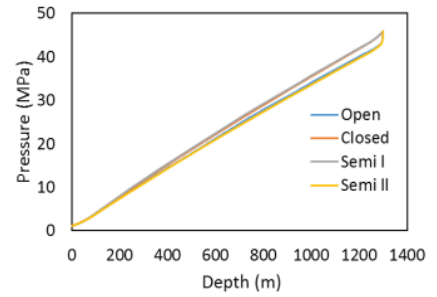

Figure 3-5. FS2 (model not including the presence of HCBF). (a-h) The concentration of fracturing fluid dissolved chemicals at the aquifer base at the end of the injection period for each investigated parameter range, and (i) pressure profile along the fault zone for four "base-case" sub-scenarios.

\subsubsection{Relative importance of parameters}

The results presented in Figure 3-4 and Figure 3-5 show the influence of each key parameter on the upward flow of fracturing fluid to the aquifer. To calculate the relative importance of parameters, the difference between the resulting concentrations at the aquifer base corresponding to the minimum and maximum parameter range values was calculated using equation 3-4. We then calculated the mean value $\left(M E_{\Delta I}\right)$ using the $\Delta I$ value from all eight sub-scenarios. $M E_{\Delta I}$ represents the influence of each key parameter on the transport distance of fracturing fluid to overlying layers, independent of the scenario and the applied boundary conditions. In the following equation, $\mathrm{C}$ represents fracturing fluid concentration at the aquifer base and $n$ is the number of sub-scenarios.

$$
\begin{aligned}
& \Delta I=(C)_{\max }-(C)_{\min } \\
& M E_{\Delta I}=\frac{\sum_{n=1}^{n=8} \Delta I_{n}}{8}
\end{aligned}
$$

The relative impact of each parameter is shown in Figure 3-6. A positive value represents an increase in concentration at the aquifer base, while a negative value represents the decrease. Fault permeability is the most influential parameter, as its increase led to a significantly higher 
vertical migration to the overburden. The injection pressure was the second most influential parameter, with a distinct correlation with the vertical extent of fluids. Overburden thickness, the third most important factor, has an inverse effect on the concentration distribution. The potential contamination threat to the aquifer decreases with the increase of the gas formation depth. Furthermore, an increase in overburden permeability and overburden anisotropy ratio causes a decrease in concentration at the aquifer base due to the increase in the horizontal spreading of fluids into overburden layers. According to Figure 3-6, fault properties are the most influential parameters dictating the upward migration of fluids into the overlying strata. The modelling results demonstrate the inverse effect of salinity on the concentration distribution at the aquifer. This strengthens the argument that high salinities in sedimentary basins decrease the potential for upward migration of brine and fracturing fluid into shallow aquifers. Based on the simulation results, the short-term contamination threats from hydraulic fracturing in deep hydrocarbon formations is minimal.

(a)

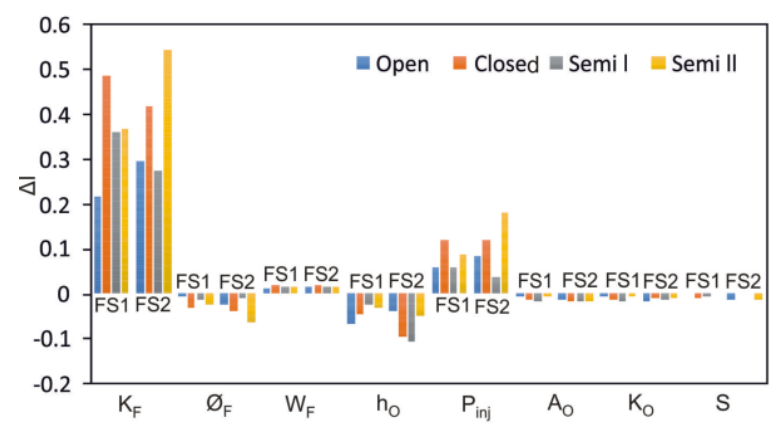

(b)

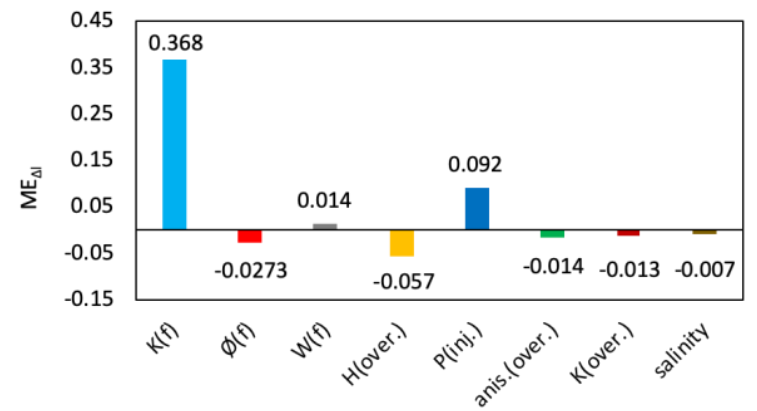

Figure 3-6. The ranked impacts of the key parameters on the migration of fracturing fluid along the fault towards the aquifer. (a) Parameter importance of the individual sub-scenarios (equation 3-4) and (b) the calculated mean value of parameter importance of all sub-scenarios (equation 3-5) for each parameter.

\subsection{Conclusions}

In this chapter, two main scenarios are selected based on an FEP approach to investigate fracturing fluid flow along a fault zone into shallow aquifers during and after hydraulic fracturing. To assess the effects of boundary conditions on the modelling results, each scenario is divided into four sub-scenarios, including: (1) open; (2) closed; (3) semi 1; (4) semi 11. A sensitivity analysis was performed to explore the relative influence of reservoir properties and 
operational parameters on the upward flow of fluids into shallow aquifers. The simulation results showed fault properties, and particularly fault permeability, are the most influential parameters inducing migration of contaminants from a shale reservoir along a fault zone. The injection pressure, reservoir depth, and overburden properties also played important roles in the upward flow of fluids. The results demonstrated that without the presence of a continuous vertical fault zone, the fluid does not reach the aquifer. The probability of contamination of shallow aquifers by upward migration of fracturing fluid through a fault zone is insignificant during and after the operation. However, the fluid concentration in the aquifer may increase for certain hydraulic conditions (e.g., high fault permeability, and porosity). Even though, fracturing operations are designed so that the stimulated reservoir volume and its adjacent hydraulically influenced areas do not intersect conductive faults, such possibility cannot be neglected. This possibility may occur either as a result of undetected geological features (e.g., insufficient resolution of the geo-physical characterization, dormant faults which are reactivated during stimulation), or as human error in the designing or conduction of the operations. This study is generic, demonstrating that quantitative risk assessment can be performed on the basis of Source-Pathway-Receptor and FEP approaches by means of forward modelling. Nevertheless, for a particular field study, reliable data acquisition must be conducted. 


\section{Chapter 4}

\section{Fracturing fluid migration along an abandoned well}

This chapter is based on the following paper:

Modeling fate and transport of hydraulic fracturing fluid in the presence of abandoned wells

Reza Taherdangkoo*, Alexandru Tatomir, Tega Anighoro, Martin Sauter

${ }^{*}$ Corresponding author

Department of Applied Geology, Geosciences Center, University of Goettingen, Goldschmidtstr. 3, D-37077 Göttingen, Germany

Citation: Taherdangkoo, R., Tatomir, A., Anighoro, T. and Sauter, M., 2019. Modeling fate and transport of hydraulic fracturing fluid in the presence of abandoned wells. Journal of contaminant hydrology, 221, pp.58-68. 


\begin{abstract}
This chapter explores the long-term (> 30 years) flow and transport of fracturing fluids into overburden layers and groundwater aquifers through a leaky abandoned well, using the geological setting of North German Basin as a case study. A three-dimensional model consisting of 15 sedimentary layers with three hydrostratigraphic units representing the hydrocarbon reservoir, overburden, and the aquifer is built. The model considers one perforation location at the first section of the horizontal part of the well, and a discrete hydraulic fracture intersecting an abandoned well. A sensitivity analysis is carried out to quantify and understand the influence of a broad spectrum of field possibilities (reservoir properties, overburden properties, abandoned well properties and its proximity to hydraulic fractures) on the flow of fracturing fluid to shallower permeable strata. The model results suggest the spatial properties of the abandoned well as well as its distance from the hydraulic fracture are the most important factors influencing the vertical flow of fracturing fluid. It is observed that even for various field settings, only a limited amount of fracturing fluid can reach the aquifer in a long-term period.
\end{abstract}




\subsection{Introduction}

Unconventional hydrocarbon reservoirs such as shale/tight gas reservoirs are characterized by low permeabilities and even natural fractures do not provide efficient pathways for fluid flow from reservoir to production wells (Reagan et al., 2015). The development of unconventional reservoirs requires stimulation techniques such as hydraulic fracturing aimed at creating artificial fracture networks and increasing local permeability (Lange et al., 2013). The process of hydraulic fracturing is characterized by injecting fracturing fluid through perforated locations in a wellbore at a bottom-hole pressure higher than the minimum principal stress (Shapiro et al., 2006; Tatomir et al., 2018). Hydraulic fracturing enhances local permeability by creating new fractures, improving fracture-fracture interaction, re-opening and further propagation of pre-existing fractures (Wang et al., 2016).

Hydraulic fracturing operations could imply a variety of environmental risks (Vengosh et al., 2014). A common concern is the upward migration of contaminants (i.e. fracturing and formation fluids) from hydrocarbon bearing formations towards shallow aquifers (Lange et al., 2013; Tatomir et al., 2018). However, the upward migration of contaminants is generally constrained by the sedimentary basin characteristics such as thickness of low permeable overburden and a low vertical hydraulic gradient, the driving force. Furthermore, the vertical movement of contaminants is bounded by the density of formation fluids and the limits of hydraulic fracturing (e.g. injection pressure, and operation duration), this in turn affects fracture aperture growth, fracture propagation, pressure propagation and stimulated volume of the reservoir (Flewelling and Sharma, 2014; Kreitler, 1989). The presence of natural preferential pathways such as faults and fractures or anthropogenic pathways such as leaky wells and a driving force are vital components that can inadvertently affect the upward migration of fluids (Flewelling and Sharma, 2014).

The majority of unconventional oil and gas fields have a long history of conventional hydrocarbon production and exploration (e.g. Pennsylvania) (Rozell, 2014). In oil and gas fields with a high density of production wells, hydraulic fractures may intercept aged abandoned wells or their vicinity (frac hit) (Brownlow et al., 2017). The probability of frac hit to an abandoned well primarily depends on depth of the horizontal well, its location and the extent of hydraulic fractures (Montague and Pinder, 2015). If frac hit materializes, depending 
on the well hydraulic characteristics, rapid vertical transport of fluids to surface or groundwater resources may occur (Lacombe et al., 1995; Reagan et al., 2015).

In hydrocarbon field development, wells are abandoned owning to unsuccessful operations or they are abandoned at reservoir depletion (Nordbotten et al., 2004). The standard practice employed in well abandonment procedure includes; emplacing cement plugs in the wellbore, with the aim of isolating different intervals including water and hydrocarbon bearing zones. The wellbore barrier system comprises a cement sheath located along the annulus between the outer casing and host rock, cement-outer casing bond, and cement-host rock bond (Gasda et al., 2013; Manceau et al., 2015; Radonjic and Kupresan, 2014). Wellbore integrity could be impaired by poor well construction (i.e. improper cementing and completion), cement degradation, casing failure, and formation damage around the wellbore. The outside of the casing could be damaged from production and injection activities during the lifetime of the well. All of the above named failure scenarios could provide pathways for vertical fluid flow into overlying layers (Carroll et al., 2016; Gasda et al., 2013). However, the most probable source of contaminant transport is through permeable flow paths outside the casing (Dusseault and Jackson, 2014).

Well abandonment practice varies from well to well and depends on several factors such as well depth, well diameter, casing condition, casing material, hydrological setting, and local regulations (Aller, 1990; Kelm and Faul, 1999). In order to properly evaluate the probability of contaminant leakage through an abandoned well, a detailed description of well completion and abandonment operations must be considered. Data needed for such evaluation could be provided from field and laboratory experiments (Gasda et al., 2013).

Many wells in mature oil and gas fields were drilled and abandoned before today's rigorous plugging standards (Evans, 2009; Nordbotten et al., 2004). In addition, many of old abandoned wells remain unplugged (Gass et al., 1997; Kang et al., 2015), and adequate documentation on their locations and spatial characteristics are rarely available (Nordbotten et al., 2004). Unplugged or improperly plugged abandoned wells provide preferential pathways for transport of fluids to the overlying aquifers; thus, poses threats to groundwater systems (Brownlow et al., 2016; Reagan et al., 2015; Tatomir et al., 2018). 
A number of research projects have investigated groundwater contamination resulting from shale gas development. However, a few studies addressed the potential threat from leaky abandoned wells. The objective of this study is to build a three-dimensional field-scale generic model to explore the long-term flow of fracturing fluid through an abandoned well as affected by fracturing operation (Figure 4-1). The reservoir of interest is the Posidonia Shale of the North German Basin. We study a wide range of field configurations and rank the significance of key parameters such as abandoned well permeability and its proximity to hydraulic fractures.

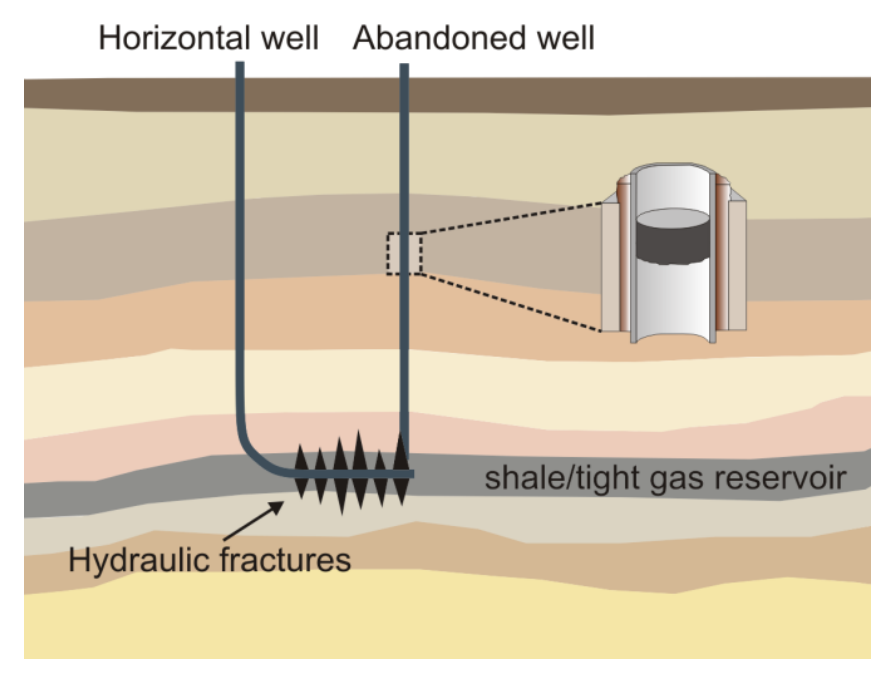

Figure 4-1. A schematic of the hydraulic fracturing intersecting an abandoned well.

\subsection{The North German Basin}

The reservoir of interest is situated within the North German Basin (NGB), which is part of the Central European Basin system (Walter, 2007). The development of the NGB system was initiated in the Late Carboniferous to Permian, owing to rifting, subsidence, and associated volcanism subsequent to the Variscan Orogenesis (Betz et al., 1987; Ziegler, 1990). The reservoir is the organic-rich Lower Jurassic Posidonia Shale. The domain consists of 15 layers of varying thickness that include three hydrostratigraphic zones representing the hydrocarbon reservoir, overburden, and aquifer. The depth information and hydraulic parameters for each layer are listed in Table 4-1. 
Table 4-1. Summary of basic parameters used in the model (Pfunt et al., 2016).

\begin{tabular}{|l|c|c|c|c|}
\hline Layer no. & Lithostratigraphic units & Thickness $(\mathrm{m})$ & Vertical permeability $\left(\mathrm{m}^{2}\right)$ & Porosity \\
\hline 1 & Quaternary sand & 50 & $5 \times 10^{-12}$ & 0.3 \\
\hline 2 & Tertiary fine sand & 50 & $5 \times 10^{-13}$ & 0.2 \\
\hline 3 & Tertiary Rupelian clay & 50 & $1 \times 10^{-17}$ & 0.1 \\
\hline 4 & Tertiary silt/clay & 50 & $1 \times 10^{-15}$ & 0.1 \\
\hline 5 & E. Cretaceous claystone & 450 & $1 \times 10^{-17}$ & 0.1 \\
\hline 6 & E. Cretaceous Wealden & 350 & $1 \times 10^{-16}$ & 0.1 \\
\hline 7 & L. Jurassic marlstone 1 & 100 & $1 \times 10^{-15}$ & 0.1 \\
\hline 8 & L. Jurassic sandstone & 200 & $1 \times 10^{-14}$ & 0.15 \\
\hline 9 & L. Jurassic marlstone 2 & 50 & $1 \times 10^{-15}$ & 0.1 \\
\hline 10 & M. Jurassic claystone 1 & 100 & $1 \times 10^{-17}$ & 0.05 \\
\hline 11 & M. Jurassic sandstone 1 & 30 & $1 \times 10^{-14}$ & 0.15 \\
\hline 12 & M. Jurassic claystone 2 & 70 & $1 \times 10^{-17}$ & 0.05 \\
\hline 13 & M. Jurassic sandstone 2 & 30 & $1 \times 10^{-14}$ & 0.15 \\
\hline 14 & M. Jurassic claystone 3 & 120 & $1 \times 10^{-17}$ & 0.05 \\
\hline 15 & Posidonia shale & 35 & $1 \times 10^{-19}$ & 0.01 \\
\hline
\end{tabular}

\subsection{Model setup and descriptions}

A three-dimensional model with a vertical dimension of $1735 \mathrm{~m}$, lateral extent of $2500 \mathrm{~m}$, and width of $1200 \mathrm{~m}$ is constructed to study flow and transport of fracturing fluid to overlying layers through a leaky abandoned well. The graphical illustration of the conceptual model is presented in Figure 4-2. The sedimentary layers are homogeneous and depth-varying relationships were applied to calculate temperature and salinity throughout the domain to better reflect the uniqueness of the study area. The average ground temperature in Germany is $8.2^{\circ} \mathrm{C}$ (Schulz et al., 2007), assuming an average geothermal gradient of $0.04{ }^{\circ} \mathrm{C} / \mathrm{m}$ (Kissinger et al., 2014). The temperature gradient induces differences in fluid density thus driving groundwater flow (Saiers and Barth, 2012). The average vertical salinity gradient of $0.15 \mathrm{~g} / \mathrm{lm}$, which is in the range reported for the NGB (Kissinger et al., 2017; Magri et al., 2005; Tesmer et al., 2007; Wolfgramm et al., 2011; Wolfgramm and Seibt, 2008), is considered in the entire domain. We do not take into account the lateral variation of salinity; therefore, any regional or local change in salinity gradients is ignored. The atmospheric pressure is assigned to the top of the model domain. A constant-head pressure boundary, which incorporates hydrostatic pressure and reservoir pressure gradient, is imposed along the lateral sides of the domain. The initial steady-state condition in the overburden and reservoir layer is calculated in the same manner as the side pressure boundary conditions. A no-flow boundary is set at bottom of the domain. Overpressure is a common feature of shale gas formations which is mainly due to the 
combination of very low permeability and the generation of significant internal gas (Soltanzadeh and Hawkes, 2009), hence the shale is considered overpressurized with a pressure gradient equal to $13 \mathrm{kPa} / \mathrm{m}$ (Gassiat et al., 2013). The overburden and reservoir pore fluid properties (e.g. density and viscosity) depend upon certain factors such as temperature, pressure, and fluid composition (e.g. salt concentration in the water phase). The density (function of $\mathrm{P}, \mathrm{T}$, salinity) and dynamic viscosity (function of $\mathrm{T}$, salinity) of the brine are calculated using Batzle and Wang (1992) equations.

This study does not reflect the conditions that normally are expected under real operation conditions. The total fracturing fluid injection volume is commonly divided fractures along the horizontal well. However, we consider the assumption that these fractures intersect a preexisting network of fractures, or a fault zone so that the fracturing fluid is concentrated in a single fracture. Therefore, we do not model a stimulated reservoir volume (SRV) but rather a fully developed discrete fracture within the shale reservoir is considered. The fracture is modeled as a lower dimensional geometric object. A straight planar height-fixed fracture, confined, and horizontally extending through the reservoir layer is assumed. The hydraulic fracture has a half-elliptic cross-section, strike of $N 45^{\circ} E$, and dip of $45^{\circ}$ towards southwest. The abandoned well hits the middle of the hydraulic fracture plane. The well is centered vertically in the domain, having a higher permeability compared to the adjacent formation. We modeled the abandoned well and the entire fractured/degraded formation parallel to the casing as a planer fracture, with a rectangular cross section. The planar fracture has a width of $0.5 \mathrm{~m}$, and thickness of $0.14 \mathrm{~m}$ corresponding to a circular cross section of a well with radius of $0.15 \mathrm{~m}$.

Hydraulic fracturing is usually conducted in multiple stages to produce fracture networks. For each stage, a certain proportion of the well casing is perforated for connecting the well to the reservoir. The highly pressurized fracturing fluid containing proppants, and other additives is then injected into the reservoir through the perforated area to create the fracture network (King, 2012). This model however does not incorporate an actual operation in terms of fracture propagation, dilatation and fracture network creation. The model assumes a perforation location at the first segment of the horizontal well from which, fracturing fluid is injected into the reservoir. The perforation location is directly connected to the fracture, and hence the fluid is directly injected in the fracture. 
Fracturing fluid is a water-based chemical mixture comprising a base fluid, friction reducers, scale inhibitors, corrosion inhibitors, surfactants, and other additives (Kekacs et al., 2015). The model considers fracturing fluid components as a pseudo-component (i.e. a conservative tracer) dissolved in the water phase. This means the model does not account for sorption and biological/chemical degradation processes. We acknowledge that modelling fracturing fluid as an inert tracer represents a conservative (less favorable) scenario and leads to an overestimation of contamination distribution in the aquifer (Kissinger et al., 2013). The fluid is tracked by means of the concentration of its dissolved chemicals. In addition, this model takes into account buoyancy resulting from the density difference between fracturing fluid and the brine. Schwartz (2015) suggests that through a certain transport distance, the probability of simultaneous migration of liquid and methane gas is relatively low owing to the contrast in their physical and chemical properties. Therefore, this study does not consider multiphase processes that require full representation of fluid and gas migration. The focus is solely on the transport of a water-based fluid in a fully saturated porous media. Fracturing fluid flow through the domain is calculated using the single phase version of Darcy's law. The system contains a single-phase flow of non-compressible fluids which consists of two components (i.e. brine and a tracer) with the exception of gas phase. The model neglects the interaction of fracturing fluid with the porous media. The numerical tool COMSOL Multiphysics 5.2a is utilized in developing and implementing the mathematical model. COMSOL uses finite element approach in solving the partial differential equations, and it is applicable for representing and accommodating complexities of real reservoir geometry and its geological objects. The time dependent solution of the equations is accomplished through a fully coupled solution approach with small time steps.

A complex, unstructured, free-triangular finite element grid was developed for discretization of the domain. The applied mesh influences the precision and computational scale, thus making it a vital component in the finite element modelling. In practice, the compatibility of the mesh density and computing capacity should be considered for the solution to satisfy the precision requirement. The abandoned well and its neighboring area were assigned a finer mesh to better simulate the upward movement of fluids through the permeable pathway and to avoid numerical problems due to large permeability contrasts. As determined by precision 
requirement, the mesh gets coarser towards boundaries of the model. The adopted mesh allowed explicitly the capture of distinctive fluid flow features in the domain.

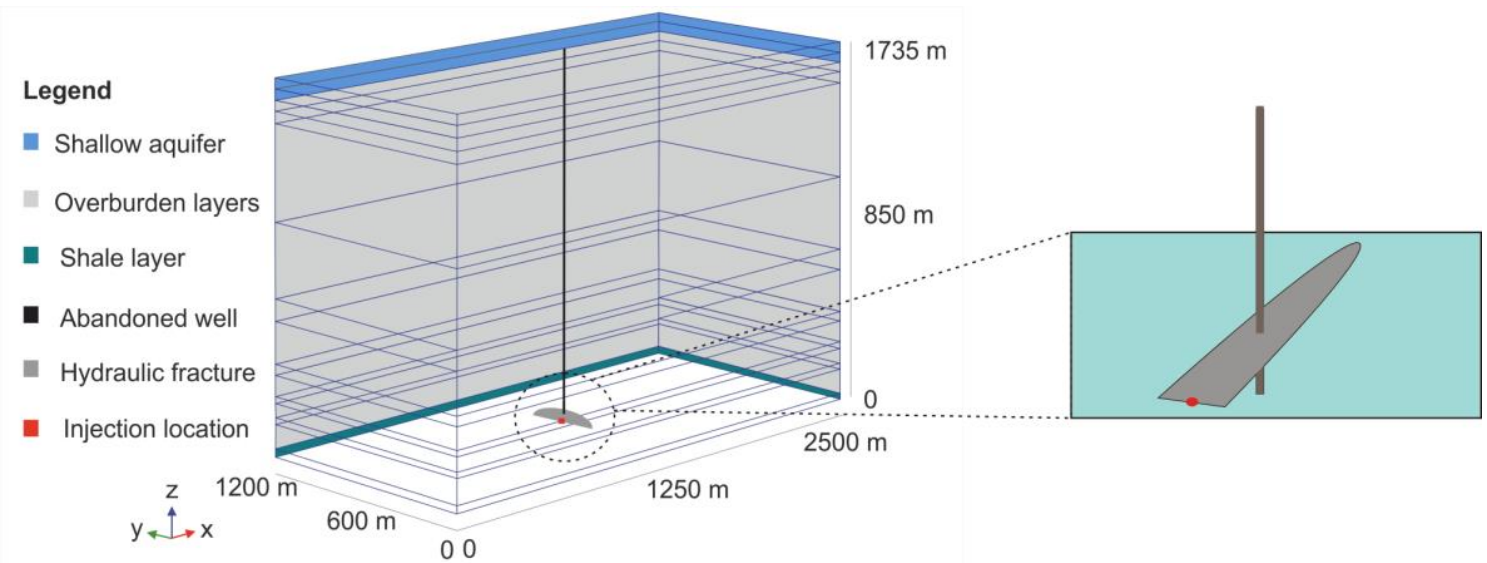

Figure 4-2. The conceptual model setup with local resolution at the fracture-well interface.

\subsubsection{Base case scenario}

The base case scenario considers geological setting of the North German Basin to systematically explore the leakage of contaminants from a shale gas reservoir through a leaky abandoned well into shallow aquifers. To realistically represent the lifetime of a typical horizontal well, each simulation comprises six periods, namely: (1) reservoir initial condition prior to fracturing; (2) injection of fracturing fluid into the reservoir; (3) shut-in period; (4) first production period; (5) second production period; and (6) post-production period. The first period models the steady state initial conditions through the system prior to operation. Afterwards, the model utilizes the initial conditions generated from the first run to simulate the injection of fracturing fluid. During the injection period, the fluid is injected into the reservoir from the perforation location at a constant rate of $3.15 \mathrm{~m}^{3} / \mathrm{min}$ for 2.5 days (Birdsell et al., 2015a). This is equivalent to injection volumes of $11356 \mathrm{~m}^{3}$ (Brownlow et al., 2016). Stage 3 represents a 5-day shut-in period, in which there is neither injection nor abstraction of fluid in the domain. Afterwards, first and second productions periods are modeled to last interruptedly for 2 and 13 years, respectively. The production rate is $16 \mathrm{~m}^{3} / \mathrm{d}$ during the first production period, and it is lowered to $3.2 \mathrm{~m}^{3} / d$ in the second period. The second production period represents the production decline throughout life of the well. In stage 6, the production is stopped and no fluid enters or leaves the domain. This stage models the continued migration of fracturing fluid into the shallow aquifers until 15 years after the production period. 
The parameters values selected for the base scenario are chosen to better understand the leakage of fracturing fluid through an abandoned well and may not realistically reflect an actual fracturing operation. Table 4-2 summarizes the input parameters for the numerical simulations. The data needed for modelling the abandoned well properties (e.g., permeability) are gathered from literature relating to modelling of fracturing fluid, methane, and $\mathrm{CO}_{2}$ leakage. The parameters for sensitivity analysis were selected based on results from literature, aimed at revealing the most influential factors determining upward migration of fluids in the subsurface. Table 4-3 presents the base case parameters and their ranges of variations for the parametric study.

Table 4-2. The general model input parameters.

\begin{tabular}{|l|c|l|}
\hline Parameter & Value & Source \\
\hline Domain length, $m$ & 2500 & \\
\hline Domain width, $m$ & 1200 & \\
\hline Overburden thickness, $m$ & 1600 & (Pfunt et al., 2016) \\
\hline Shale thickness, $m$ & 35 & (Pfunt et al., 2016) \\
\hline Abandoned well porosity & 0.15 & (Celia et al., 2005; Ebigbo et al., 2007) \\
\hline Surface temperature, ${ }^{\circ} \mathrm{C}$ & 8.2 & (Schulz et al., 2007) \\
\hline Salinity gradient, $g /(l . m)$ & 0.15 & (Wolfgramm and Seibt, 2008) \\
\hline Geothermal gradient, ${ }^{\circ} \mathrm{C} / \mathrm{m}$ & 0.04 & (Kissinger et al., 2014) \\
\hline Hydraulic fracture half-length, $m$ & 150 & (Chaudhary et al., 2011; Economides and Nolte, \\
\hline Hydraulic fracture width, $m$ & 0.08 & (Economides and Nolte, 1989; Shapiro et al., 2006) \\
\hline Hydraulic fracture permeability, $\mathrm{m}^{2}$ & $1 \times 10^{-11}$ & (Economides and Nolte, 1989) \\
\hline Hydraulic fracture porosity & 0.3 & (Shapiro et al., 2006) \\
\hline $\begin{array}{l}\text { Molecular diffusivity of solutes in } \\
\text { pure fluid, } \mathrm{m}^{2} / \mathrm{s}\end{array}$ & $1 \times 10^{-9}$ & (Pfunt et al., 2016) \\
\hline Water compressibility, $1 / \mathrm{Pa}$ & $4.4 \times 10^{-}$ & (Pfunt et al., 2016) \\
\hline Fracturing fluid density, $\mathrm{kg} / \mathrm{m}^{3}$ & 1000 & (Gassiat et al., 2013; Pfunt et al., 2016) \\
\hline Longitudinal dispersivity, $\mathrm{m}$ & 10 & (Pfunt et al., 2016) \\
\hline Transverse dispersivity, $\mathrm{m}$ & 1 & (Pfunt et al., 2016) \\
\hline
\end{tabular}

Table 4-3. Model parameters and range.

\begin{tabular}{|l|c|c|c|l|}
\hline Parameter & Unit & value & Variation & Source \\
\hline $\begin{array}{l}\text { Abandoned well } \\
\text { permeability, } K_{a}\end{array}$ & $m^{2}$ & $\begin{array}{c}1 \times 10- \\
12\end{array}$ & $\begin{array}{c}1 \times 10^{-17}- \\
1 \times 10^{-11}\end{array}$ & $\begin{array}{l}\text { (Bai and Reinicke, 2013; Celia et al., 2005; } \\
\text { Ebigbo et al., 2007; Gasda et al., 2008; Nogues } \\
\text { et al., 2011; Nordbotten et al., 2005, 2004; } \\
\text { Pawar et al., 2009) }\end{array}$ \\
\hline $\begin{array}{l}\text { Shale permeability, } \\
K_{s}\end{array}$ & $m^{2}$ & $\begin{array}{c}1 \times 10- \\
19\end{array}$ & $\begin{array}{c}1 \times 10^{-21}- \\
1 \times 10^{-18}\end{array}$ & $\begin{array}{l}\text { (Brownlow et al., 2016; Neuzil, 1994; Reagan et } \\
\text { al., 2015) }\end{array}$ \\
\hline
\end{tabular}




\begin{tabular}{|c|c|c|c|c|}
\hline Shale porosity, $\emptyset_{s}$ & & 0.01 & $0.01-0.05$ & $\begin{array}{l}\text { (Freeze and Cherry, 1979; Gassiat et al., 2013; } \\
\text { Neuzil, 1994) }\end{array}$ \\
\hline $\begin{array}{l}\text { fracturing fluid } \\
\text { volume, } V_{F F}\end{array}$ & $m^{3}$ & 11356 & $\begin{array}{c}11000- \\
15000\end{array}$ & $\begin{array}{l}\text { (Birdsell et al., 2015a; Brownlow et al., 2016; } \\
\text { Myers, 2012a) }\end{array}$ \\
\hline $\begin{array}{l}\text { Fracture-well } \\
\text { proximity }\end{array}$ & $m$ & & $5-15$ & \\
\hline $\begin{array}{l}\text { Reservoir } \\
\text { overpressure } \\
\text { gradient, }(d P / d z)_{R}\end{array}$ & $\begin{array}{l}\text { KPa } \\
/ \mathrm{m}\end{array}$ & 13 & $10-17$ & $\begin{array}{l}\text { (Brownlow et al., 2016; Gassiat et al., 2013; } \\
\text { Sumi, 2008) }\end{array}$ \\
\hline $\begin{array}{l}\text { Overburden } \\
\text { thickness, } B_{o}\end{array}$ & $m$ & 1600 & $900-2900$ & (Gassiat et al., 2013; US EIA, 2011b, 2011a) \\
\hline $\begin{array}{l}\text { Overburden } \\
\text { permeability, } K_{O}\end{array}$ & $m^{2}$ & & $\begin{array}{l}1 \times 10^{-17}- \\
1 \times 10^{-15}\end{array}$ & $\begin{array}{l}\text { (Gassiat et al., 2013; Gleeson et al., 2011; } \\
\text { Luijendijk and Gleeson, 2015) }\end{array}$ \\
\hline Salinity, S & $\mathrm{g} / \mathrm{lm}$ & 0.15 & $0.1-0.2$ & (Kissinger et al., 2017; Magri et al., 2005) \\
\hline
\end{tabular}

\subsubsection{Parametric study}

A parametric study is designed for a wide spectrum of field configurations to examine the extent of fracturing fluid migration from the gas formation into the shallower strata. The impact of injection rate, reservoir and overburden properties, salinity, and abandoned well properties is evaluated. The parametric study applies one-at-a-time (OAT) method (Morris, 1991) in analyzing the influence of changing the values of each chosen parameter. OAT method has a limited use in exploratory modelling as it does not explore all the possible combinations between input variables, and it usually provides a local sensitivity measurement. However, OAT is a simple, easy to implement, and computationally cheap method, thus providing relevant observations as it exposes the independent effects of each parameter. The North German Basin geology is considered as the baseline where each parameter is changed at a time.

\subsubsection{Fracturing fluid injection rate}

The effectiveness of hydraulic fracturing treatment depends on several factors such as injection rate, injection depth, injection pressure, and fracturing fluid properties (e.g. viscosity). Wang et al. (2016) concluded that fluid injection rate influences treating pressure, corresponding stimulated reservoir volume and hydrocarbon production. We changed injection rate to capture the full range of its potential influence on fracturing fluid flow. The injection rate varies between 3.05 to $4.16 \mathrm{~m}^{3} / \mathrm{min}$, thus corresponding to a total injection volume of between 11000 and $15000 \mathrm{~m}^{3}$ (Birdsell et al., 2015a; Brownlow et al., 2016; Myers, 2012a). 


\subsubsection{Shale properties}

Shale gas formations possess specific features such as heterogeneity, low matrix porosity and permeability (Wang et al., 2015; Zhu et al., 2012). The formation characteristics play important roles in gas production from shale gas reservoirs. The effective porosity and permeability reduce during production due to pore pressure reduction within the reservoir. However, we consider constant values for reservoir porosity and permeability during the entire simulation period. We examined the effect of porosity and permeability of the rock matrix over a range reported in literature. The porosity and permeability variation range from 1 to $5 \%$ and $1 \times 10^{-21}$ to $1 \times 10^{-18} \mathrm{~m}^{2}$, respectively.

\subsubsection{Shale overpressure}

Overpressure occurs when pore fluid pressure is higher than normal hydrostatic fluid pressure at a specific depth (Bowers, 2002). The overpressure in subsurface rocks is produced through three main mechanisms, namely: increase in compressive stress, change in pore fluid volume, and fluid buoyancy. The disequilibrium compaction and gas generation are the most probable mechanisms for producing pressure anomalies in low permeable sediments (Osborne and Swarbrick, 1997). Disequilibrium compaction is a common overpressuring mechanism in thick low permeable sequences (e.g. shale, and clay) with continued rapid burial. Overpressure is a transient phenomenon except when it is due to topography (Ligtenberg, 2005; Osborne and Swarbrick, 1997). In our model, the overpressure is considered as an initial condition prior to fracturing (Birdsell et al., 2015a). We applied overpressure only within the shale, and not in its overlying formations. We acknowledge that the applied approach does not reflect the actual overpressure within the shale. The overpressure varies from 10 to $13 \mathrm{kPa} / \mathrm{m}$ (Brownlow et al., 2016; Gassiat et al., 2013) to understand its effect on migration of fracturing fluid into shallow layers.

\subsubsection{Overburden properties}

The depth and physical properties of overburden layers play a critical role in flow and transport of fracturing fluid towards shallower strata (Gassiat et al., 2013; Taherdangkoo et al., 2017). The depth to shale gas formations in sedimentary basins varies between 1 to $4.5 \mathrm{~km}$, with an average depth of $2.9 \mathrm{~km}$ (Gassiat et al., 2013; US EIA, 2011b). The permeability of sedimentary layers also changes over a wide range (Gleeson et al., 2011). The significance of these parameters was evaluated through a scenario which considers one overburden layer 
with averaged values calculated from the NGB data. The overburden thickness ranges from 900 to $2900 \mathrm{~m}$ and overburden permeability changes from $1 \times 10^{-17}$ to $1 \times 10^{-15} \mathrm{~m}^{2}$.

\subsubsection{Salinity}

Salinity increases with depth in sedimentary basins (Ferguson et al., 2018). A change in the initial distribution of salinity could influence the vertical flow of fracturing fluid (Taherdangkoo et al., 2017). We assumed three different salt distributions within the domain prior to the operation to understand its significance in the upward migration of fracturing fluid. The vertical salinity gradient of $0.10,0.15$, and $0.20 \mathrm{~g} / \mathrm{lm}$ are considered.

\subsubsection{Abandoned wellbore integrity}

There is limited information available on the quality of the sealing materials used for old abandoned wells, and hence the hydraulic parameters (e.g. permeability) of the degraded barrier system is largely unknown (Nogues et al., 2011). Previous modelling studies suggested a range of effective permeability for evaluating the vertical migration of fluids through a leaky wellbore (Bai and Reinicke, 2013; Celia et al., 2005; Ebigbo et al., 2007; Gasda et al., 2008; Nogues et al., 2011; Nordbotten et al., 2005, 2004; Pawar et al., 2009). Herein, the effective wellbore permeability is the primary parameter of interest and it averages the permeability of the entire fractured/degraded formation parallel to the casing. We consider a wide range of possible wellbore permeabilities based on the wellbore integrity of old wells that were drilled completed, and abandoned, using different technologies. The model is run to simulate a 30year period under the assumption that wellbore properties of the abandoned well remained constant through the entire simulation period. An open wellbore has permeability values between $2 \times 10^{-13}$ and $1 \times 10^{-11} \mathrm{~m}^{2}$ while in a properly sealed well permeabilities span between $1 \times 10^{-17}$ to $1 \times 10^{-13} \mathrm{~m}^{2}$.

\subsubsection{Proximity of fracturing to abandoned well}

Frac hit is a common occurrence in oil and gas fields with a high density of production wells, and even multiple frac hits may materialize (Lawal et al., 2013). However, in some cases, the abandoned well may not be affected by fracturing. The abandoned well can be close or at a large distance from the operation. Therefore, it is important to explore the influence of the abandoned well proximity to hydraulic fractures on the upward flow of fluids to shallower 
aquifers. The study is conducted by varying the lateral distance of the abandoned well from the fracture. The fracture-well proximity is varied between 5 to $15 \mathrm{~m}$.

\subsubsection{Influence of well production}

Hydrocarbon production affects fluid flow behavior within the hydrocarbon bearing formation (Brownlow et al., 2016; Flewelling and Sharma, 2014). Production leads to large scale depressurization in the reservoir which drives fluid to flow downward rather than upward. The influence of well production on the upward flow of fluids is explored through a scenario which does not consider production from the horizontal well. In this case, fracturing fluid remains in the domain during the entire simulation period. The aim is to explore the long-term contaminant distribution, in the absence of an active production well.

\subsection{Results and discussion}

\subsubsection{Base case scenario}

The spatial distribution of the plume in the y-z plane at $x=1250 \mathrm{~m}$ at the end of injection and shut-in periods is shown in Figure 4-3. The spread of fracturing fluid within the fracture, flow through the abandoned well, and its rise through the overburden layers is displayed. The red arrows display the direction of total fluid flow in the domain. Figure 4-4 compares the concentration of fracturing fluid dissolved chemicals along the abandoned well at the end of each simulation period. The $y$-axis represents depth of the domain from the aquifer base to bottom of the reservoir. The Posidonia shale and its overlying layer (i.e. M. Jurassic claystone 3) host the bulk of fluid during the first two periods. The vertical migration of the fluid is relatively low and it is primarily due to the shale overpressure, fracturing pressure, and upward buoyancy. The induced overpressure is dissipated in the reservoir during the shut-in period. However, the reservoir pressure remains higher than the conditions prior to injection. As apparent from Figure 4-3, the total fluid flow direction is upward at the end of the injection period and it is mostly downward at the end of the shut-in period. Fracturing fluid reaches a maximum height of $252 \mathrm{~m}$ from fracture-well intersection (i.e. depth of $1468 \mathrm{~m}$ from the top) at the end of the injection period, and it increases to a height of $263 m$ (i.e. $1457 m$ depth) at the end of the shut-in period. The short-lived shut-in period has a minimal influence on the upward movement of fluids. The shape of the plume at the end of injection period is identical 
to that for the shut-in period. Therefore, in Figure 4-4a, the iso-concentration lines belonging to these periods overlapped. Note that the flow of fluids in each layer depends on the layer physical properties (e.g. permeability). The strata heterogeneity decreases the upward movement of fracturing fluid through the abandoned well.

(a)

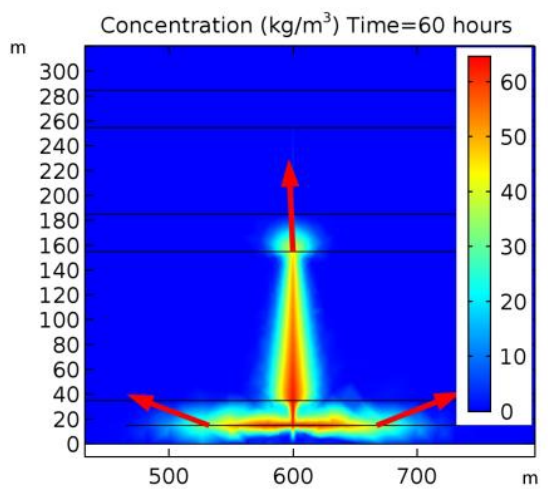

(b)

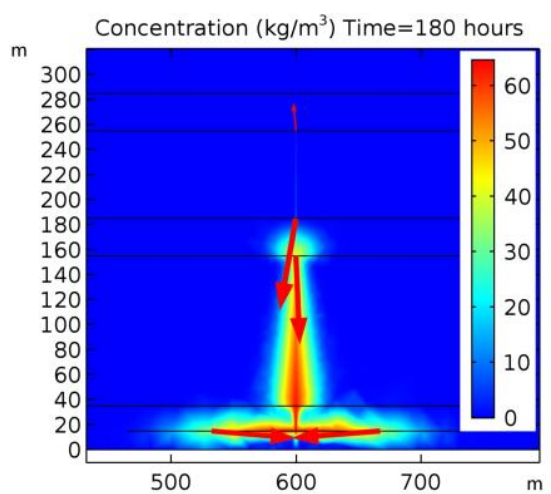

Figure 4-3. The distribution of fracturing fluid in the y-z plane at $x=1250 \mathrm{~m}$ at the end of (a) injection and (b) shut-in period.

(a)

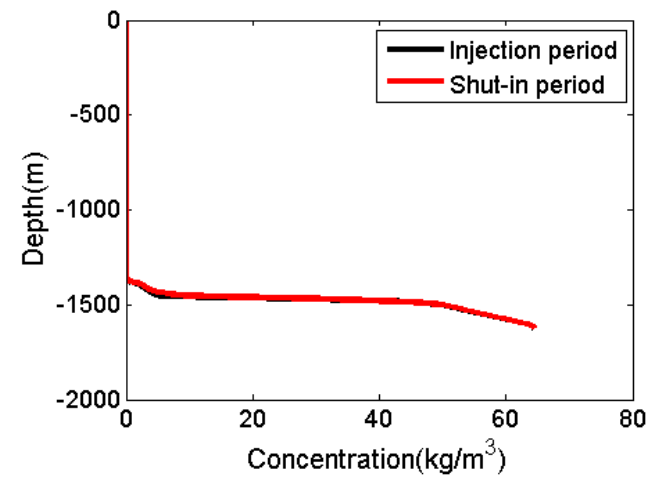

(b)

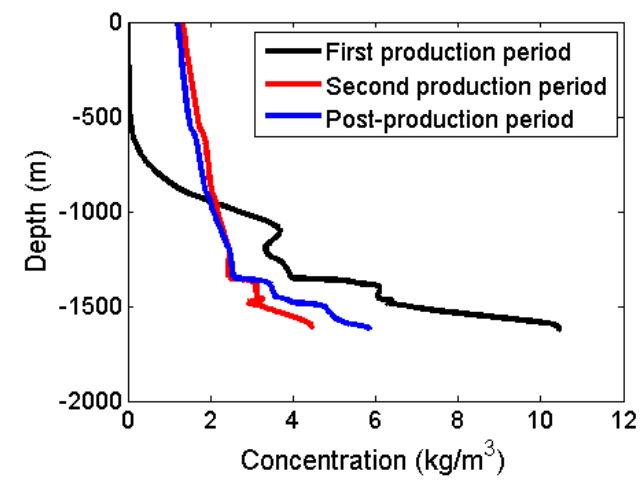

Figure 4-4. The concentration of fracturing fluid dissolved chemicals along the abandoned well in the $y-z$ plane at the end of (a) injection and shut-in periods and (b) first production, second production and post-production periods.

Fracturing fluid is subject to upward buoyancy during the entire simulation which is due to density differences between the injected fluid and brine. The induced overpressure is removed from the reservoir at the beginning production. Fracturing fluid does not reach the aquifer at the end of the first production period (Figure 4-5), and it reaches a maximum height of $1250 \mathrm{~m}$ from the fracture-well intersection (i.e. depth of $470 \mathrm{~m}$ from the top boundary). The upward flow rate is low due dissolution of fracturing fluid in formation water, and the lateral fluid flow in overburden layers. At the end of stage 3, the concentration of dissolved chemicals at 
fracture-well interface is $10.5 \mathrm{~kg} / \mathrm{m}^{3} ; 6$ times lower than the concentration at the end of stage 2. The spatial distribution of the plume in the subsurface is displayed in Figure 4-6.

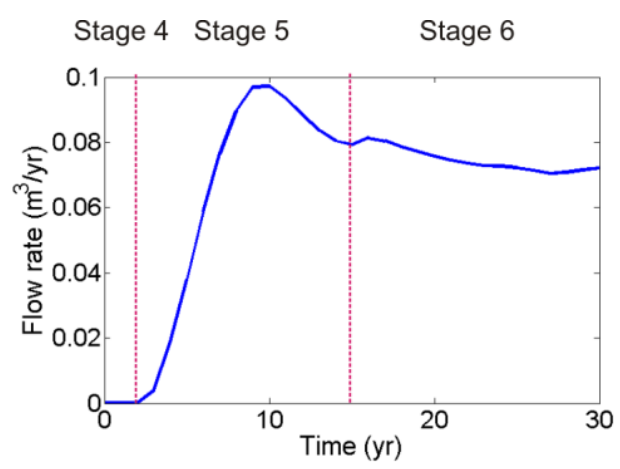

Figure 4-5. Fracturing fluid flow rate along the abandoned well at the overburden-aquifer interface during the simulation time.

Fracturing fluid flow rate at the aquifer base is negligible prior to 3 years of production. The fluid reaches the shallow freshwater aquifer one year after the commencement of the second production period. Fracturing fluid flow rate into the aquifer peaked in tenth year, and afterwards experienced a steady decline. The flow rate at the overburden-aquifer interface is $0.08 \mathrm{~m}^{3} / y \mathrm{r}$ at the end of production. During production, $67 \%$ of the injected fluid volume is extracted from the domain; hence, production plays a vital role in inhibiting substantial vertical migration of fluids toward shallow aquifers. The production causes large-scale depressurization within the reservoir that in turn draws fluids downward rather than upward. The upward buoyancy force decreases over time due to dissolution of fracturing fluid with brine during its transport. Fracturing fluid extraction from the domain, its lateral spreading into the overburden layers and decrease in the buoyancy force explain the decrease in the upward fluid flow along the abandoned well during production and post-production periods.

(a)

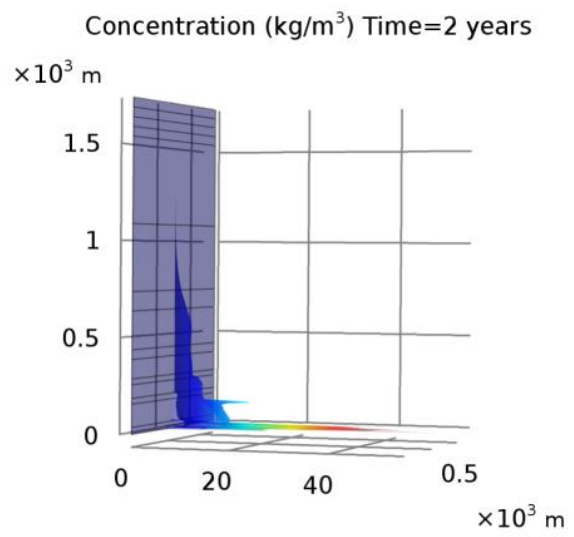

(b)

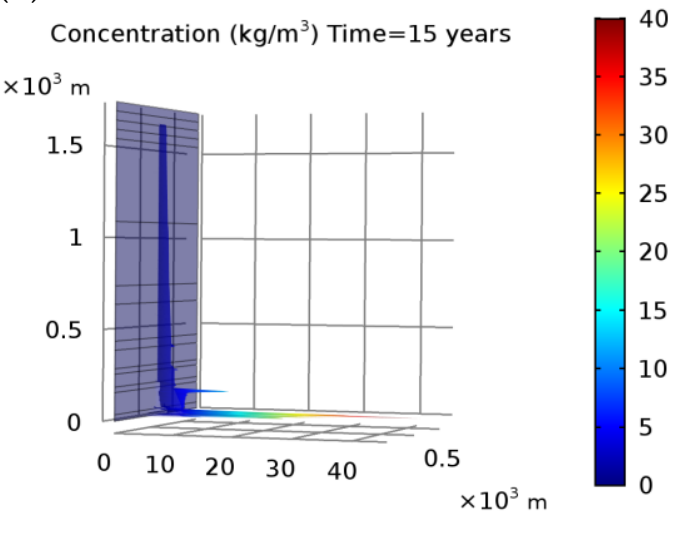


(c)

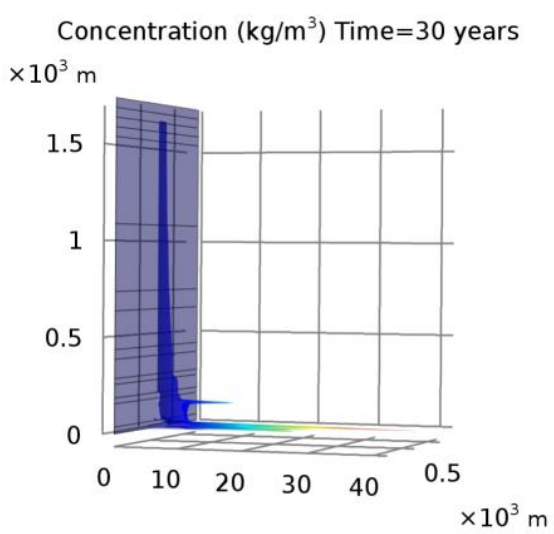

Figure 4-6. Spatial distribution of fracturing fluid along the abandoned well and its neighboring area at the end of (a) first production, (b) second production, and (c) post-production period. The cut plane is located at $\mathrm{y}-\mathrm{z}$ direction at $x=1250 \mathrm{~m}$.

The pressure build-up occurs in the Posidonia shale after end of the production. The upward flow rate through the abandoned well is much lower during the post-production period. At the end of the simulation, $67 \%$ of fracturing fluid volume is discharged by production, $33 \%$ remained in the subsurface, and $0.02 \%$ reached the shallow aquifer. Figure $4-6 \mathrm{c}$ shows the distribution of the plume through the well and the overburden layers at the end of simulation periods (30 years). Fracturing fluid flow rate along the abandoned well at the overburdenaquifer interface is $0.07 \mathrm{~m}^{3} / \mathrm{yr}$ at the end of simulation.

\subsubsection{Parametric study}

The sensitivity analysis is designed to examine field uncertainties and draw a meaningful conclusion for the defined failure scenario. A total of 30 simulations, aimed at assessing different field scenarios were ran by altering the key parameter values. Figure 4-7 illustrates fracturing fluid flow rate at the overburden-aquifer interface during 30 years of simulation time.

The total volume of fracturing fluid in the system correspondingly increases with the increase in the injection rate. Thus, a higher amount of fluid remains in the subsurface even after the end of production. A higher injection rate imposes a higher induced pressure in the reservoir. Therefore, the pressure front propagates a greater distance within the formation. This increases the upward flow of fluids through the abandoned well consequently results in more contamination in shallower strata. Figure $4-7 a$, presents fracturing fluid rate at the 
overburden-aquifer interface based on the total injected fluid volume $\left(V_{F F}\right)$. When the injection rate increases to $4.16 \mathrm{~m}^{3} / \mathrm{min}$ (fluid volume of $15000 \mathrm{~m}^{3}$ ), the upward fluid flow into the aquifer is 1.75 times higher when compared to the base case scenario (fluid volume of $11356 \mathrm{~m}^{3}$ ). Modelling results indicate that higher injection rates lead to a slightly higher chemical concentration in the shallow aquifers. Hence, the long-term influence of the injection volume on the upward movement of fluids to overlying layers is relatively low.

The effect of shale properties on fracturing fluid flow are shown in Figure 4-7 (b-c). The ascent of fluids through abandoned well decreases with an increase in the shale permeability. This occurs because pressure dissipation across the abandoned well decreases with an increase in the reservoir permeability. However, it leads to slight changes in the vertical fluid flow at overburden-aquifer interface. A reduction in the shale porosity also decreases the upward fluid flow. It mirrors the same effect that reservoir permeability has on vertical flow of fluids. The amount of extracted fracturing fluid by the well decreases with the decrease in shale permeability. The corresponding volume is $76 \%$ and $63 \%$ for shale permeability of $1 \times 10^{-18}$ and $1 \times 10^{-21} \mathrm{~m}^{2}$, respectively. This shows that fluid flow into overlying aquifers decreases with the increase in fracturing fluid discharge. The changes in shale porosity have insignificant influence on discharge of fracturing fluid from the domain. Although the physical properties of the reservoir affect the upward flow of fluids to shallower layers, their significance on the long-term concentration distribution in shallower layers is negligible.

During the short-lived injection period, the reservoir overpressure and fracturing injection pressure are the dominant forces for upward fluid flow. Subsequently, large-scale depressurization pressure occurs over lifetime of the well. Thus, in the defined failure scenario, the reservoir overpressure only has a short-term influence on the vertical flow of fluids. The long-term upward migration of the flow through the abandoned well is not dependent on the shale overpressure (Figure 4-7d). The result indicates that the overpressure gradient does not have influence on the extracted volume of fracturing fluid from the domain during the production period. Note that the significance of shale overpressure on the vertical flow of fluids highly depends on the type of boundary conditions applied (Birdsell et al., 2015a; Taherdangkoo et al., 2017). Thus, the obtained results regarding the effect of shale overpressure may change by applying different initial and boundary conditions. 
The depth of shale gas formation is an important factor affecting the vertical flow of fluids. Fracturing fluid reaches the aquifer base one year after the operation for an overburden depth of $900 \mathrm{~m}$. In this case, the flow rate at the overburden-aquifer interface is two times larger compared to the base case model. Fracturing fluid could not be detected in the aquifer for overburden depth of 2400 and $2900 \mathrm{~m}$ (Figure 4-7e). The probability of aquifer contamination is slightly higher in shallower shale formations. The flow rate at the aquifer base increases with an increase in the permeability of overburden layer (Figure 4-7f). Fracturing fluid flow rate into the aquifer is negligible for an averaged overburden permeability of $1 \times 10^{-17} \mathrm{~m}^{2}$.

A change in the initial distribution of salinity gradient influences the vertical flow of fluids in the domain (Figure 4-7g). For a salinity gradient of $0.1 \mathrm{~g} / \mathrm{lm}$, the flow rate at the aquifer base is $0.08 \mathrm{~m}^{3} /$ year and the discharge volume during production is $68 \%$ of the initial volume. For a salinity gradient of $0.2 \mathrm{~g} / \mathrm{lm}$, the flow rate is $0.05 \mathrm{~m}^{3} /$ year and the discharge volume is $66 \%$.

The upward migration of fluids through the abandoned well strongly depends on the effective wellbore permeability (Figure 4-7h). The fluid does not migrate upward for wellbore permeabilities equal to or lower than $1 \times 10^{-13} \mathrm{~m}^{2}$. In this case, fracturing fluid does not reach the shallow aquifers even after 30 years. For well permeability of $1 \times 10^{-17} \mathrm{~m}^{2}$, the volume of fracturing fluid discharged from the model domain by production is $75 \%$ of the initially injected volume and the remaining $25 \%$ of fracturing fluid volume is confined deep in the subsurface, far from groundwater aquifers. Hence, abandoned wells with low permeabilities (i.e. high wellbore integrity) prevent upward fluid flow into overburden layers. Figure 4-7h illustrates that a slight change in the wellbore permeability leads to a significant change of flow rate at the overburden-aquifer interface, thus resulting in an increase in chemical concentration in the aquifer. For instance, when the wellbore permeability is $2 \times 10^{-12} \mathrm{~m}^{2}$, the corresponding flow rate at the overburden-aquifer interface is 3.6 times higher compared to the base case. The maximum flow rate into the aquifer is observed for a well permeability of $1 \times 10^{-11} \mathrm{~m}^{2}, 27.4$ times higher than that for the base case scenario. In addition, $56 \%$ of the injected fluid volume is extracted from the model domain during production period, $1 \%$ reaches the aquifer, and $43 \%$ is confined in the subsurface. The modelling results suggest that wellbore integrity largely controls the flow of fluids into the shallower strata and consequently the contamination distribution into the aquifer. The results indicate that the abandoned well has to fail to act as a permeable pathway for flow of fluids. 
Increasing the fracture-well distance significantly influences fracturing fluid arrival at the aquifer base. When the fracture-well distance is $5 \mathrm{~m}$, the plume reaches the shallow aquifer 4 years after production (Figure 4-7i). At the end of simulation, the flow rate at the overburdenaquifer interface is 3.6 times lower when compared to the base case. An increase in fracturewell distance to $10 \mathrm{~m}$ reduces the fluid flow to $0.01 \mathrm{~m}^{3} / \mathrm{yr}$. This value is 7.2 times lower compared to the base case model. When the production well is $5 \mathrm{~m}$ away from the hydraulic fracture, $74 \%$ of injected fluid volume is extracted during production. This value remains constant for a fracture-well distance of 10 and $15 \mathrm{~m}$ which is due to the minimal effect of the hydraulic fracture to the abandoned well. When the abandoned well is $15 \mathrm{~m}$ away from the fracture, fracturing fluid is not detected in the aquifer. The model results indicate that the proximity of hydraulic fracture to the abandoned well is the second most influential parameter on the vertical flow of fluids to shallower strata. When well-fracture distance is large enough, most of fracturing fluid is discharged from the domain and the possibility of upward flow is largely inhibited.

The scenario that does not consider production leads to a higher contaminant distribution in the shallow aquifers (Figure 4-7j). At the end of simulation, the flow rate into the aquifer is 10 times higher compared to the base case. This indicates the production from the reservoir significantly reduces contamination treats to groundwater aquifers.

The modelling results suggest that the wellbore integrity of the abandoned well and its distance from fracturing operation are the most influential parameters determining the vertical flow of fluids through an abandoned well. The fluid injection rate and overburden and reservoir properties also have impacts on vertical fluid flow towards shallow aquifers. It is evident that the plume does not reach the aquifer within a short-time frame. The modelling results show limited vertical transport of fluids through the abandoned well under different field possibilities. However, a thorough evaluation of hydrogeological conditions and production history of a suggested field is required prior to hydraulic fracturing operation to minimize the risk to groundwater aquifers. 
(a)

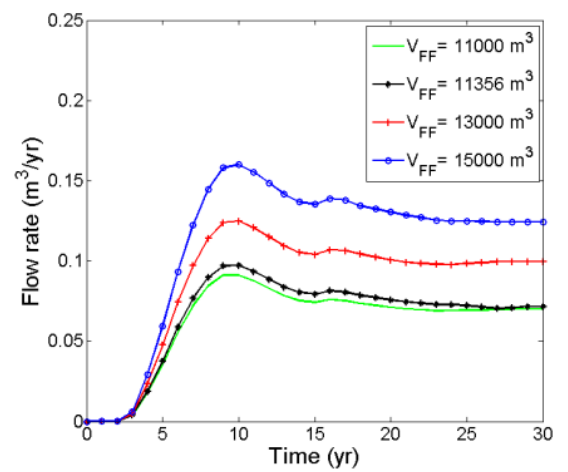

(c)

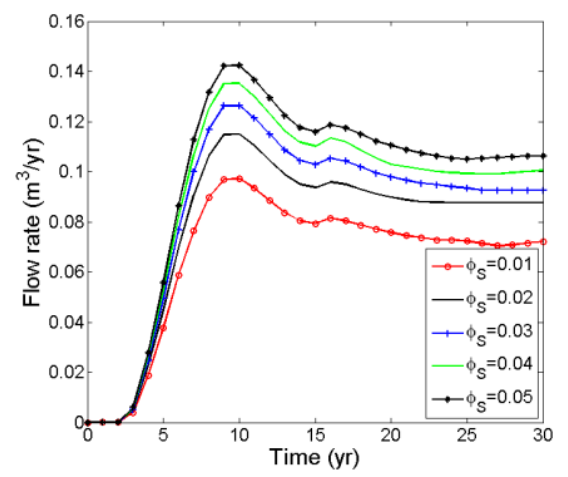

(e)

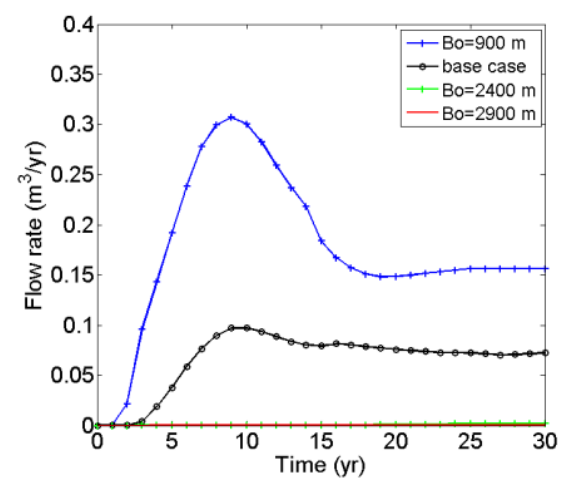

(g)

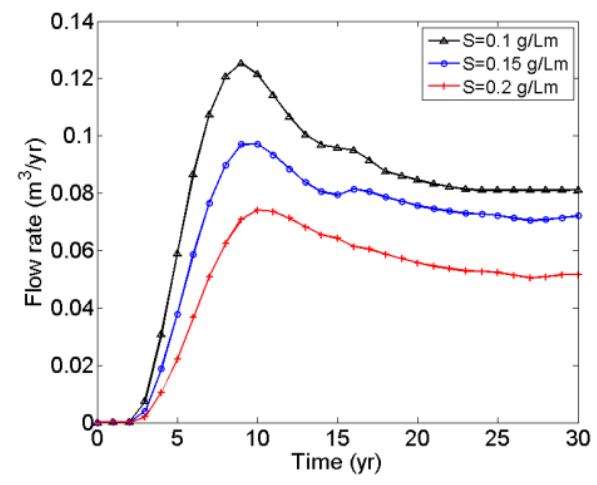

(b)

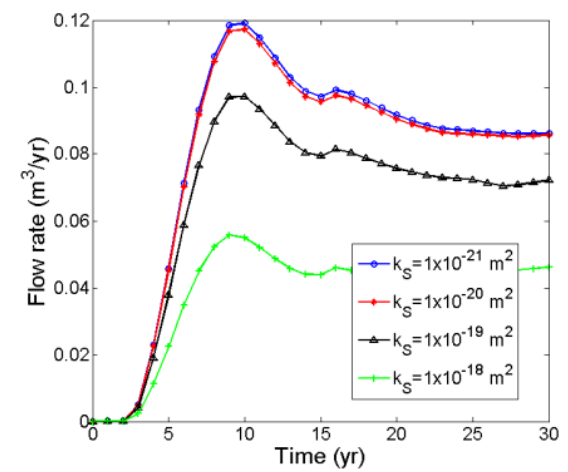

(d)

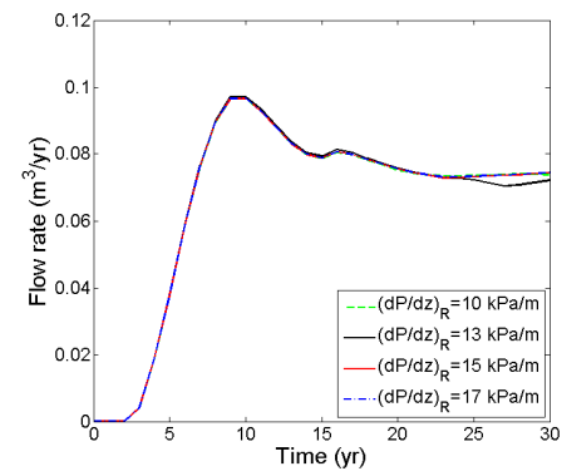

(f)

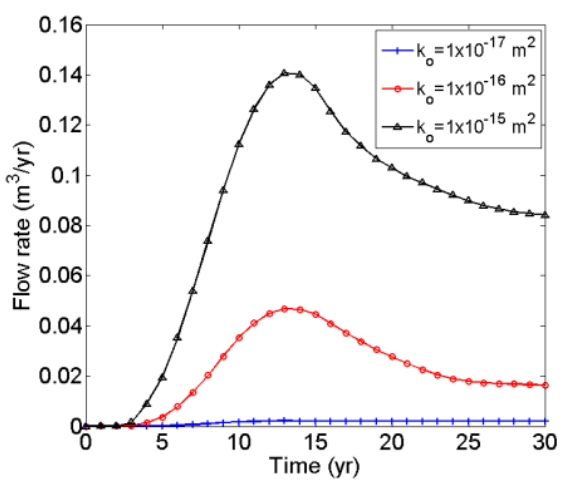

(h)

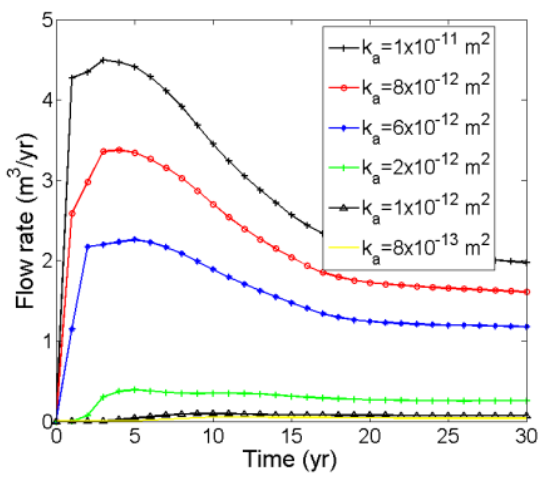


(i)

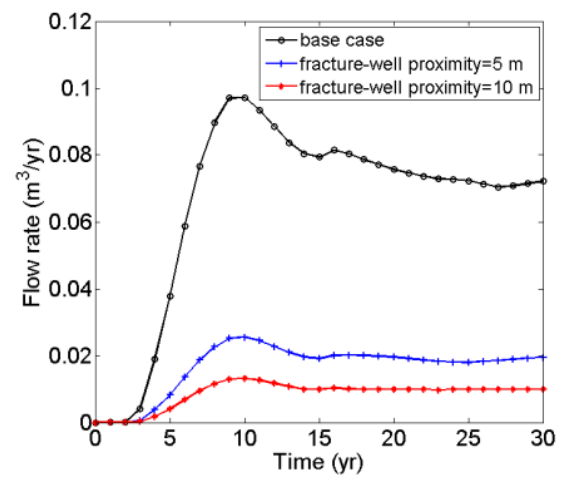

(j)

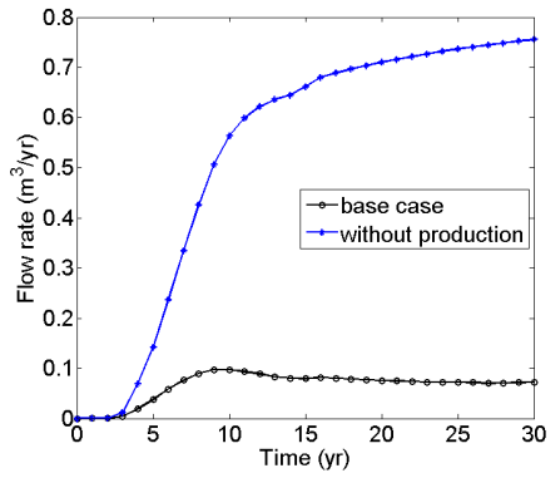

Figure 4-7. Fracturing fluid flow rate along the abandoned well at the overburden-aquifer interface during the entire simulation time. (a) Injection volume, (b) shale permeability, (c) shale porosity, (d) shale overpressure gradient, (e) overburden thickness, (f) overburden permeability, (g) salinity gradient, (h) abandoned well permeability, (i) fracture-well proximity, and (j) the scenario without considering the well production.

\subsection{Conclusions}

This research focuses on the numerical simulation of fracturing fluid flow and transport from a hydrocarbon bearing formation towards shallow aquifers through an abandoned well. The models are based on permeability and stratigraphy data of the geological sequence in the North German Basin. We conducted a parametric study to examine the influence of key factors, such as overburden and reservoir properties, abandoned well permeability and its proximity to hydraulic fractures on the vertical flow of fracturing fluid through an abandoned well. Numerical modelling results revealed the following:

- During the injection period, fracturing pressure and reservoir overpressure are the main driving forces for vertical movement of fracturing fluid. During the shut-in period, the pressure is dissipated across the reservoir. Thereafter, the fracturing pressure is removed and large-scale depressurization occurs over time via production from the horizontal well. The upward buoyancy is an important mechanism for upward flow of fluids to shallower strata during production and post-production periods.

- The production extracts a significant amount of fracturing fluid (67\% of total volume) from the domain thus reducing the probability of upward fluid flow to overlying 
layers. When production is not incorporated in the model, the flow rate at the overburden-aquifer interface is 10 times higher than that of the base case. This indicates that neglecting the effect of well production leads to the overestimation of fluid flow through the well, and consequently contamination distribution in the aquifer.

- The influence of reservoir physical properties and the injection volume on the vertical migration of fluids is inconsequential over the long-term. The significance of shale overpressure highly depends on the type of applied boundary conditions. The strata heterogeneity decreases the upward migration of fluids through the abandoned well. The probability of upwards migration of fracturing fluid is lower in sedimentary basins with high salinity.

- The combined effect of well production and dilution of fracturing fluid with formation water significantly decreases the upward movement of fluids towards shallower strata. We suggest that further modelling studies take into account these important effects.

- Most notably, increasing abandoned well permeability significantly increases the upward fluid rate to shallow aquifers. When well permeability is set at $1 \times 10^{-11} \mathrm{~m}^{2}$, the flow rate at the overburden-aquifer interface is 27.4 times higher than the base case. Fracturing fluid does not reach the aquifers for wellbore permeability lower than $1 \times 10^{-13} \mathrm{~m}^{2}$. Therefore, wellbore integrity is considered as the most important factor controlling the leakage of fluids through an abandoned well. It is concluded that only a defected wellbore could act as a permeable pathway for movement of fluids to overlying layers.

- The proximity of fracturing to the abandoned well is the second most influential parameter influencing the upward flow of fluids. Fracturing fluid is not detected in the aquifer when the abandoned well is $15 \mathrm{~m}$ away from the hydraulic fracture. However, the findings for an abandoned well with a permeability of $1 \times 10^{-13} \mathrm{~m}^{2}$ and the fracture-well distance of $15 \mathrm{~m}$ may change with different geological settings.

- The short-term probability of shallow aquifer contamination resulting from fracturing is negligible even in the presence of a leaky abandoned well. Model results show that fracturing fluid reaches the aquifer three years after production. The long-term 
simulation reveals that the risks hydraulic fracturing pose to shallow aquifers are low (i.e. $0.02 \%$ of injected fluid reach to the aquifer at the of simulation time). 


\section{Chapter 5}

\section{NAR neural networks to predict fracturing fluid leakage into shallow groundwater}

This chapter is based on the following paper:

Reza Taherdangkoo ${ }^{1 *}$, Alexandru Tatomir ${ }^{1,2}$, Mohammad Taherdangkoo ${ }^{3}$, Pengxiang Qiu ${ }^{1}$, Martin Sauter ${ }^{1}$

${ }^{*}$ Corresponding author

${ }^{1}$ Department of Applied Geology, Geosciences Center, University of Göttingen, Goldschmidtstr. 3, D-37077 Göttingen, Germany

${ }^{2}$ Department of Earth Sciences, Uppsala University, Villavägen 16, S-75236 Uppsala, Sweden

${ }^{3}$ Department of Artificial Intelligence, Tehran Business School, Golestan St, No. 93, Tehran, 3 85433, Iran

Citation: Taherdangkoo, R., Tatomir, A., Taherdangkoo, M., Qiu, P. and Sauter, M., 2020. Nonlinear autoregressive neural networks to predict hydraulic fracturing fluid leakage into shallow groundwater. Water, 12(3), pp. 841. 


\begin{abstract}
This chapter describes the novel application of a nonlinear autoregressive (NAR) neural network to estimate fracturing fluid flow rate to shallow aquifers in the presence of an abandoned well. The NAR network is trained using the Levenberg-Marquardt (LM) and Bayesian Regularization (BR) algorithms and the results were compared to identify the optimal network architecture. For NAR-LM model, the coefficient of determination $\left(R^{2}\right)$ between measured and predicted values is 0.923 and the mean squared error (MSE) is $4.2 \times$ $10^{-4}$, and the values of $R^{2}=0.944$ and MSE $=2.4 \times 10^{-4}$ were obtained for the NAR-BR model. The results indicate the robustness and compatibility of NAR-LM and NAR-BR models in predicting fracturing fluid flow rate to shallow aquifers. This study shows that NAR neural networks can be useful and hold considerable potential for assessing the groundwater impacts of unconventional gas development.
\end{abstract}




\subsection{Introduction}

Hydraulic fracturing (HF) is a commonly applied technology for extraction and production from unconventional hydrocarbon resources. Hydraulically fractured horizontal wells are the dominant type of newly drilled oil and natural gas wells (Cook et al., 2018). The potential impacts of the technology on drinking water resources have gained substantial attention in the water-energy nexus and have been described and discussed for some time (Sauter et al., 2012; Tatomir et al., 2018; Vengosh et al., 2014). Of particular interest are the potential impact of fracturing fluids on groundwater quality because they are difficult to assess, due to limited available data and the complex nature of multiphase flow and transport processes in the subsurface (Lange et al., 2013; Rice et al., 2018a).

Migration of formation fluid and brine from a shale gas reservoir to overlying strata could occur through natural or anthropogenic permeable pathways, such as faults, fractures, and bedding planes and leaky abandoned wells, in the vicinity of fracturing operations (Birdsell et al., 2015a; Gassiat et al., 2013; Myers, 2012a; Taherdangkoo et al., 2017). Abandoned wells could be affected by frac hits, i.e., the interaction between hydraulic fractures and wells, and the steep pressure gradient associated with the operation (King et al., 2017). The frac hit to an abandoned well may facilitate upward fluid migration, depending on the cement/wellbore integrity (Gasda et al., 2004). The preferential flow pathways along oil and gas abandoned wells are associated with poor well construction, cement degradation, casing failure, and formation damage around the wellbore. The formation of preferential flow pathways, the increase of cement effective permeability, and the wellbore proximity to hydraulic fracturing operations enhance the risk of upward fluid flow to shallow groundwater (Brownlow et al., 2016; Gasda et al., 2013, 2004; Taherdangkoo et al., 2019).

The presence of low-permeability overburden rocks (Flewelling and Sharma, 2014; Sauter et al., 2012), production from the horizontal well (Birdsell et al., 2015a; Brownlow et al., 2016; Taherdangkoo et al., 2019), fracturing fluid imbibition into the shale reservoir (Birdsell et al., 2015b; Edwards et al., 2017), and mixing or other dilution processes during the transport (Pfunt et al., 2016; Taherdangkoo et al., 2019) limit the vertical extent of fracturing fluid, thus reducing the contamination threat to shallow groundwater. Osborn et al. (Osborn et al., 2011) analyzed water samples from water wells in aquifers overlying northeastern Pennsylvania (active HF region) and New York (HF is currently not allowed). The authors found no relationship 
between contamination of groundwater samples and fracturing fluid or deep brine. However, a number of modelling studies suggest that a small amount of fracturing fluid could reach the aquifer under special conditions, i.e., the presence of connective permeable pathways, such as faults and leaky abandoned wells (Brownlow et al., 2016; Gassiat et al., 2013; Kissinger et al., 2013; Taherdangkoo et al., 2019).

To properly assess the groundwater contamination potential from unintended migration along abandoned wells over a long period, detailed databases on water quality and wellbore integrity are needed (Cao et al., 2020; Rice et al., 2018a; Taherdangkoo et al., 2019). Data-driven models are particularly useful in this area because surface and groundwater-quality data are sparse, particularly at the regional scale, and computational sources are limited.

Artificial neural networks (ANNs) constitute well recognized data-driven models in the field of hydrogeology (Guzman et al., 2017; Kisi and Cigizoglu, 2007; Loucks et al., 2005) and many studies have shown that ANN models have good forecasting performance compared to some of the traditional approaches (Olyaie et al., 2015). Despite an exponential increase in the application of artificial intelligence methods within the hydrogeology research community over the past decade, they have not been applied in investigating the environmental impact of hydraulic fracturing. ANNs have gained popularity in the field of water quality prediction and forecasting due to (i) the use of non-physics based algorithm, (ii) the dealing with nonlinear relationships properly, and (iii) the experience of users that can be incorporated easily into the model structure (Maier and Dandy, 1996; Zhang and Stanley, 1997). The nonlinear autoregressive (NAR) network is a recurrent, dynamic neural network that is well suited for the prediction of time series, where there is only one series involved (Wei et al., 2012). The NAR model extracts generic principles from the past values of the time series to predict its future values (Ruiz et al., 2016; Wei et al., 2012). In general, successful application of NAR models primarily depends on (i) size, quality and universal validity of input data, and (ii) the proper model development and assessment. It has been shown that NAR networks converge much faster and generalize better in comparison with the conventional ANNs (Adamowski et al., 2012). The development and application of NAR models in the context of hydraulic fracturing need expertise from both computational and hydrogeological fields. 
We present a NAR network to predict flow rates of fracturing fluid to a shallow aquifer in the presence of an abandoned well. We trained the NAR network with the Levenberg-Marquardt and Bayesian Regularization algorithms. The results show that the NAR network is able to accurately model fracturing fluid flow rate to the aquifer. This innovative research is the first study that employs a NAR neural network for evaluating groundwater contamination from shale gas development.

\subsection{Material and Methods}

\subsubsection{Conceptual model for fracturing fluid migration along an abandoned well}

The dataset considered here includes flow rates of fracturing fluid to the aquifer overlying the Posidonia shale formation in the North German Basin, taken from Taherdangkoo et al. (2019). They studied the influence of various phases of fracturing operations on the migration of fracturing fluid from the shale into the shallow aquifer along an abandoned well. The conceptual model is shown in Figure 5-1. The three-dimensional model domain has $1700 \mathrm{~m}$ of depth, $2500 \mathrm{~m}$ of length, and $1200 \mathrm{~m}$ of width. The domain consists of 15 layers, representing the shale, overburden layers, and two aquifers. The top of the model is set to the atmospheric pressure. The lateral sides are set to constant-head boundary conditions, and the base of the model is set a no-flow boundary condition (Taherdangkoo et al., 2019).

Hydraulic fracturing is usually conducted in multiple stages, but the model considers only one perforation location at the first segment of the horizontal well from which, fracturing fluid is injected into the reservoir. The model assumes a fully developed hydraulic fracture, having a strike of $\mathrm{N} 45^{\circ} \mathrm{E}$, and a dip of $45^{\circ}$ towards the southwest, within the reservoir prior to the leakage. As illustrated in Figure 1, the perforation location is connected to the hydraulic fracture, thus fracturing fluid is directly injected into the fracture. The abandoned well hits the middle of the fracture plane and extends from the shale to the aquifers. The abandoned well and the entire fractured/degraded formation parallel to the wellbore casing are considered as a planer fracture with a rectangular cross section, having an effective permeability higher than surrounding sediments.

The model assumes single-phase flow of non-compressible fluids, consisting of two components, i.e., brine and a tracer. Fracturing fluid is considered as a pseudo-component (i.e., 
a conservative tracer) dissolved in the aqueous phase. In order to realistically assess the upward migration of fluids over the lifetime of a typical horizontal well, each simulation consists of six stages, namely, fracturing fluid injection, well shut-in, first production, second production, and abandonment. Firstly, a total volume of $11,356 \mathrm{~m}^{3}$ of fracturing fluid (Brownlow et al., 2016) is injected into the domain during 2.5 days. Then, a 5-day shut-in period takes place. Afterwards, the production periods are modeled with outflow rates of 16 and $3.2 \mathrm{~m}^{3} /$ day for an interrupted period of 2 and 13 years, respectively (Brownlow et al., 2016). In the abandonment stage, the production is stopped. The fluid flow along the abandoned well and at the overburden aquifer interface is monitored. Note that we used data reported in Brownlow et al. [14], which are based on available industry data for the Eagle Ford Shale play in south Texas.

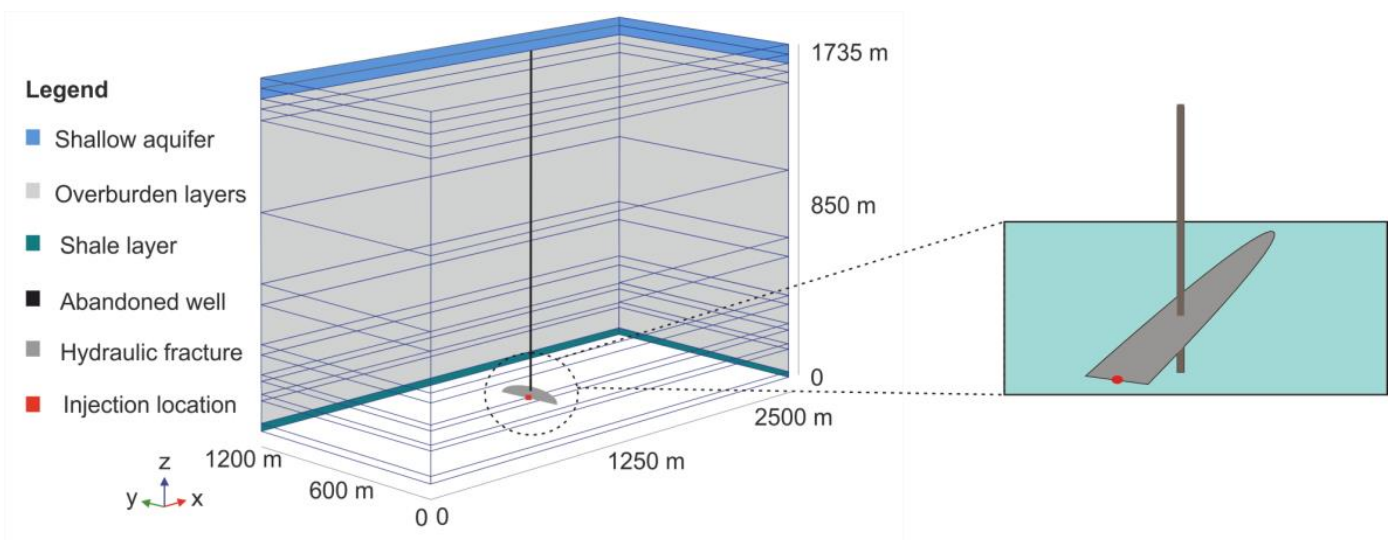

Figure 5-1. The conceptual model used for simulations.

\subsubsection{Data preparation and Analysis}

Taherdangkoo et al. (2019) conducted an extensive sensitivity analysis on the most influential parameters to examine the extent of fracturing fluid migration from the shale into the overlying aquifers along an abandoned well. They presented their results in terms of fracturing fluid flow rates to the aquifer over 30 years. The parameters studied include fracturing fluid injection rate, shale permeability, shale porosity, overburden thickness, overburden permeability, salinity, effective abandoned well permeability, and its distance to hydraulic fractures. The parameter values used in the base-case model and sensitivity analysis are shown in Table 5-1. As an example, fracturing fluid flow rate to the aquifer during the simulation time for the base-case model is displayed in As an example, fracturing fluid flow rate to the aquifer for the base-case model is displayed in Figure 5-2 (Taherdangkoo et al., 
2019). We used fluid flow rates obtained from the sensitivity analysis to build the input dataset, which includes 31-time steps of 24 elements (i.e., model runs). In total, the input dataset contains 744 data records of fracturing fluid flow rate to the aquifer.

Following the ratio of 60:20:20, the input dataset (i.e., fracturing fluid flow rate data) was randomly divided into training, validating, and testing sets. A sample of 446 data was specified for the training phase, 149 data for the validation phase, and the remaining 149 data were used to analyze the reliability and robustness of the NAR network models. The preprocessing step was performed to prepare the data for the training phase, to achieve more consistent and better results. In general, the Levenberg-Marquardt and Bayesian Regularization algorithms work best if the network inputs and feedbacks are in the range of -1 to 1 (Guzman et al., 2017). Thus, by applying a normalization function, input and feedback values were scaled in the interval $(-1,1)$ and back-transformed in the testing phase.

Table 5-1. Parameters used in base-case model and sensitivity analysis simulations.

\begin{tabular}{|l|c|c|c|c|}
\hline Parameter & Unit & Base-case value & Min. & Max. \\
\hline Shale permeability & $\mathrm{m}^{2}$ & $1 \times 10^{-19}$ & $1 \times 10^{-21}$ & $1 \times 10^{-18}$ \\
\hline Shale porosity & & 0.01 & 0.01 & 0.05 \\
\hline Overburden permeability & $\mathrm{m}^{2}$ & Depth dependent & $1 \times 10^{-17}$ & $1 \times 10^{-15}$ \\
\hline Overburden thickness & $\mathrm{m}$ & 1600 & 900 & 2900 \\
\hline Salinity gradient & $\mathrm{g} / \mathrm{lm}$ & 0.15 & 0.1 & 0.2 \\
\hline Fracturing fluid volume & $\mathrm{m}^{3}$ & 11365 & 11000 & 15000 \\
\hline Abandoned well permeability & $\mathrm{m}^{2}$ & $1 \times 10^{-12}$ & $1 \times 10^{-17}$ & $1 \times 10^{-12}$ \\
\hline Distance of fracture plane to well & $\mathrm{m}$ & 0 & 0 & 15 \\
\hline
\end{tabular}

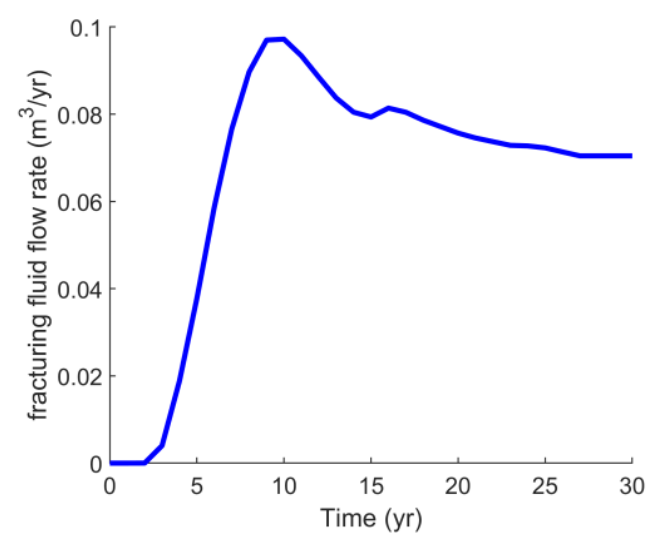

Figure 5-2. Fracturing fluid flow rate to the aquifer for the base-case model during the simulation time. 


\subsubsection{NAR Model}

The prediction of the incidence of fracturing fluid flow to shallow groundwater is a nonlinear problem. The nonlinear autoregressive (NAR) neural network represents a powerful class of models that has favorable qualities for recognizing time series patterns and nonlinear characteristics. The NAR network is a recurrent dynamic network with feedback connections enclosing layers of the network; thus, the current output depends on the values of past output (Da Costa Lopes et al., 2015). The NAR network can be applied to effectively forecasting time series and can be written as follows (López et al., 2012; Ruiz et al., 2016; Wang et al., 2017):

$$
\hat{y}=f(y(t-1)+y(t-2)+\cdots+y(t-d))+\varepsilon(t)
$$

where $f$ is an unknown nonlinear function that can be approximated by the feedforward neural networks during the training process; $\hat{y}$ is the predicted value of the data series of $y$ at a discrete time step $t, d$ represents past values of the series, and $\varepsilon(t)$ is the approximation error of the series y at time $t$. The NAR network is described in Figure 5-3.

The development of the optimal architecture for the NAR model requires determination of time delays, the number of hidden nodes, the activation function, and an efficient training algorithm. The optimum number of time delays and hidden nodes were obtained through a trial-and-error procedure (Ruiz et al., 2016). The activation function was selected based on Maier and Dandy (Maier and Dandy, 2000). Finally, Levenberg-Marquardt and Bayesian Regularization algorithms were applied to train the model.

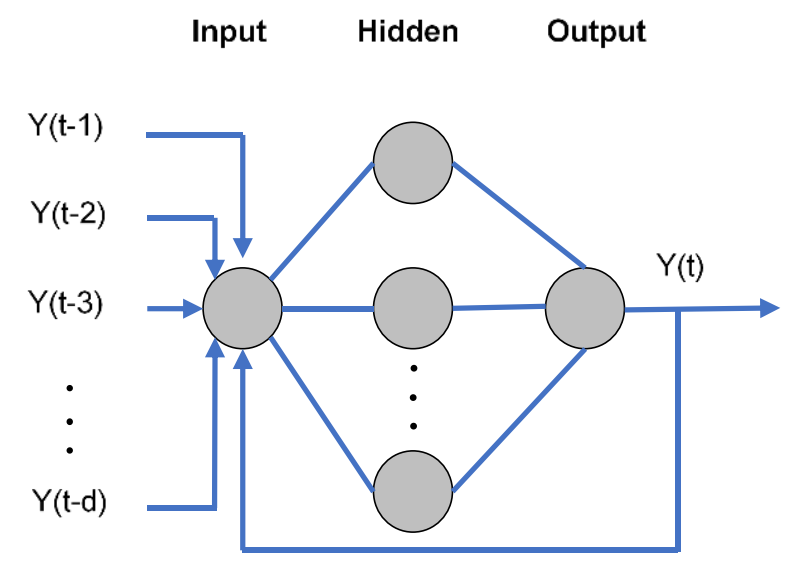

Figure 5-3. A sketch of a standard NAR neural network. 


\subsubsection{Training Algorithms}

\subsubsection{Levenberg-Marquardt}

The Levenberg-Marquardt (LM) is a backpropagation-type algorithm and has been widely used for training the NAR network because of the fast convergence speed (Hagan and Menhaj, 1994; Marquardt, 1963). The LM is an effective modification of the Gauss-Newton method that finds the function (either linear or nonlinear) minima over a space of parameters and optimizes the solution (Sahoo and Jha, 2013). The LM algorithm uses an approximation of the Hessian matrix, given by (Bishop, 1995):

$$
\Delta w=\left[\boldsymbol{J}^{T}(w) \boldsymbol{J}(w)+\lambda I\right]^{-1} \boldsymbol{J}^{T}(w) e(w)
$$

in which $w$ represents the weight, $\boldsymbol{J}$ is the Jacobian matrix, $\boldsymbol{J}^{T}$ is the transpose matrix of $\boldsymbol{J}$ and $\boldsymbol{J}^{T} \boldsymbol{J}$ is the Hessian matrix. $I$ represents the learning matrix, and $\lambda$ and $e$ are the learning coefficient and vector of network errors, respectively. The parameter $\lambda$ is automatically updated based on the error at each iteration to secure the convergence. We used a random value of $\lambda$ to initiate the iteration process for optimizing weights with the LM algorithm.

\subsubsection{Bayesian Regularization}

Bayesian Regularization (BR), introduced by MacKay (1992), is a mathematical technique for converting nonlinear systems into "well posed" problems (Burden and Winkler, 2008). The BR network reduces the potential for overfitting in the training phase while eliminating the need for the validation phase. Thus, BR is especially suitable for small datasets because more data are available for the training (Burden and Winkler, 2008; Doan and Liong, 2004). The BR automatically sets the best performance function to accomplish an efficient generalization on the basis of Bayesian inference. The determination of the optimal regularization parameters depends upon the computation of the Hessian matrix at the minimum point (Foresee and Hagan, 1997; MacKay, 1992b). In this work, a Gauss-Newton approximation of the Hessian matrix is applied to optimize regularization (Foresee and Hagan, 1997).

\subsubsection{Network Architecture}

The NAR network employed is a feed forward neural network with three layers, namely input, hidden and output layers (Figure 5-3). Sigmoid function, a continuous non-linear function, is the most commonly used activation function for neural network design with back propagation training. The activation functions in hidden and output layers are logistic sigmoid and linear, 
respectively. A trial-and-error procedure is carried out to determine the number of nodes in the hidden layer and value of time delays in order to generate accurate model responses. The Levenberg-Marquardt and Bayesian Regularization algorithms were employed for training of the NAR network and their performance were evaluated under the same network structure. The necessary steps to implement the network is illustrated in Figure 5-4.

The NAR neural network is trained in a series-parallel configuration. In the training phase, the true output is available and it was used as the input to the network. During the testing phase, the calculated output was fed back to the network to estimate the next value of the output in a parallel configuration. In this study, the initial weights in the network are assigned randomly and they were adjusted at each iteration (i.e. epoch) to reduce the error. The procedure continued until the network output met the stopping criteria.

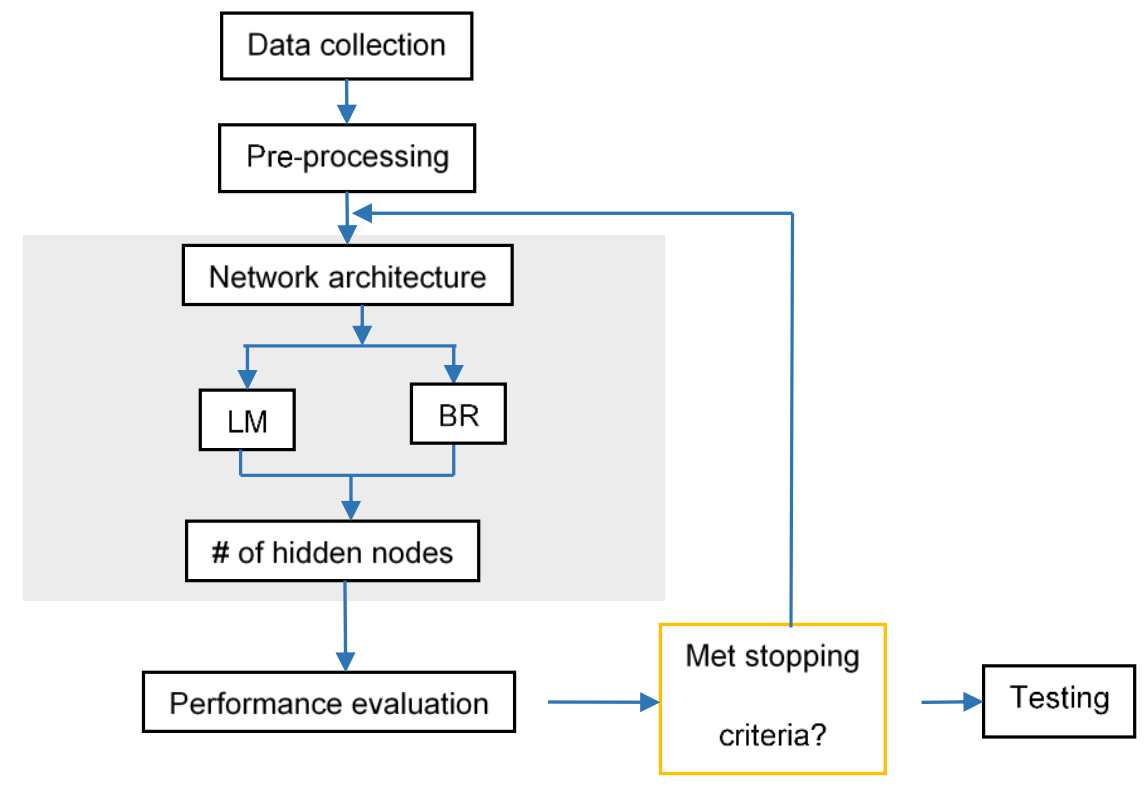

Figure 5-4. A basic procedure for the NAR network training.

\subsubsection{Performance Evaluation}

The prediction performance of the models was evaluated by two well-known statistical criteria, namely the coefficient of determination $\left(R^{2}\right)$ and mean squared errors (MSE). $R^{2}$ is a linear regression used to analyze the best fit between the measured values and model's predicted values, given by:

$$
R^{2}=1-\frac{\sum_{i=1}^{n}\left(y_{i}-\hat{y}_{i}\right)^{2}}{\sum_{i=1}^{n}\left(y_{i}-\bar{y}_{i}\right)^{2}}
$$


in which $y$ and $\hat{y}$ represent the measured and predicted values, respectively. $\bar{y}$ stands for the average of measured values and $n$ equals the number of values. The MSE calculates the average squared difference between the measured and predicted values, given by the following equation:

$$
M S E=\frac{1}{n} \sum_{i=1}^{n}\left(y_{i}-\hat{y}_{i}\right)^{2}
$$

\subsection{Results and Discussion}

This study considers the failure scenario of upward migration of fracturing fluid from the shale formation into a shallow aquifer along an abandoned well. We employed a NAR network model to predict the incident of fracturing fluid in the aquifer. The NAR network has one input, i.e., the fracturing fluid flow rate into the aquifer, and it generates one output that is the prediction of flow rate at a certain time ahead. We used the logistic sigmoid and linear activation functions at the hidden and output layers respectively because the modelling problem presented here is a function approximation problem. The error analysis showed that the network with three nodes in the hidden layer and two time delays provide the best performance.

Figure 5-5 shows the results of the error autocorrelation function of the fracturing fluid flow rate. This function describes the relationship between the prediction errors and time, and it was used to determine the value of time delays in the NAR network. To obtain a perfect training fit, there should be only one nonzero value over the entire function. As shown for the current network structure (node $=3$ and delay $=2$ ), error autocorrelations were approximately in the $95 \%$ confidence interval zone, except for the ones at zero lag, thus NAR-LM and NARBR models are adequate. The calculated MSE and $R^{2}$ for the training phase (Table 5-2) show that both models reached the best fitting performances in terms of evaluation criteria. For instance, $R^{2}$ values of higher than 0.99 indicated a strong correlation between the measured values and fitting values. According to Figure 5-5 and Table 5-2, we conclude that a sample of 446 data is adequate to train the NAR models. 
(a)

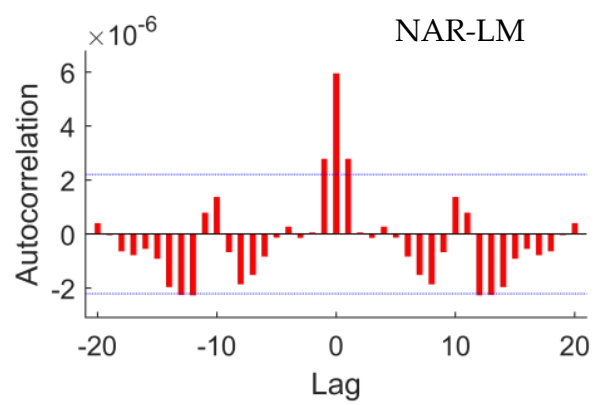

(b)

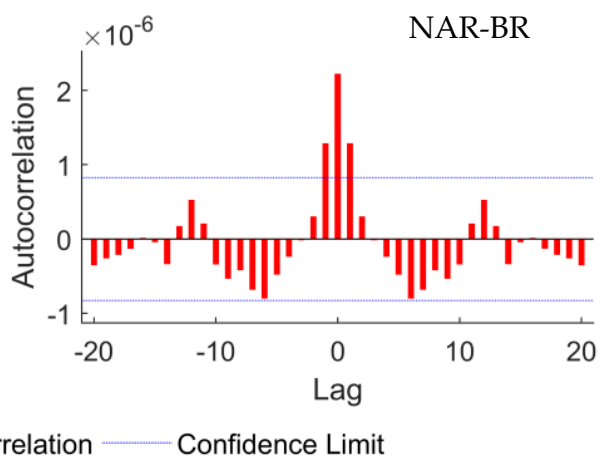

Figure 5-5. Error autocorrelations of (a) NAR-LM and (b) NAR-BR models for the training phase.

Table 5-2. Evaluation of the NAR-LM and NAR-BR models performances.

\begin{tabular}{|c|c|c|c|c|c|}
\hline Statistical parameter & \multicolumn{3}{|c|}{ NAR-LM } & \multicolumn{2}{c|}{ NAR-BR } \\
\hline & Training & validation & Testing & Training & Testing \\
\hline $\mathrm{R}^{2}$ & 0.998 & 0.996 & 0.923 & 0.996 & 0.944 \\
\hline MSE & $1.07 \times 10^{-5}$ & $1.2 \times 10^{-5}$ & $4.2 \times 10^{-4}$ & $1.3 \times 10^{-5}$ & $2.4 \times 10^{-4}$ \\
\hline
\end{tabular}

An open-loop architecture (series-parallel configuration) is more useful for training the NAR network, while a closed-loop architecture (parallel configuration) is suitable for multiple-stepahead predictions (Wei et al., 2012). We used a transfer function to convert the network into the closed-loop after finishing the open-loop training. The prediction ability of the models in terms of evaluation indices is summarized in Table 5-2. The testing results were similar to those of training: for example, $\mathrm{R}^{2}$ values higher than 0.9 for the testing phase indicated the satisfactory performance of both developed models. For further comparison of evaluation indices, MSE of NAR-LM and NAR-BAR were $4.2 \times 10^{-4}$ and $2.4 \times 10^{-4}$ respectively, which proved the strong ability of the developed models in predicting the nonlinear behavior of fracturing fluid flow to the aquifer. The results suggest that NAR-BR model has a slightly better prediction performance compared to that of the NAR-LM model in terms of larger $\mathrm{R}^{2}$ and smaller MSE.

The scatter diagrams of the measured and predicted flow rate values by NAR-LM and NARBR networks are illustrated in Figure 5-6 and Figure 5-7, respectively. We also plotted a 45degree reference line. The measured and predicted data should lie close to the reference line to demonstrate a very good model fit. For the training phase, this can be seen in Figure 5-6a and Figure 5-7a indicating both models fit equally well statistically. Although the predicted 
values of NAR-LM model fit better to the reference line, $\mathrm{R}^{2}$ value of NAR-BR model is slightly higher.

The performance plots of NAR-LM and NAR-BR models are shown in Figure 5-8. These figures illustrate the relationship between the testing, training, validation phases in predicting fluid flow rates into the aquifer in terms of MSE versus number of epochs. The black circle in Figure 5-8a shows the performance of the NAR-LM model, which performed best during validation process. The black circle in Figure 5-8b shows the performance of the NAR-BR model, which was the best one during the testing phase. As illustrated in Figure 5-8b, the best performance for the validation phase is $1.2 \times 10^{-5}$ at epoch 9 for the NAR-LM model, while the NAR-BR model experiences the best performance $\left(\mathrm{MSE}=1.3 \times 10^{-5}\right)$ for the training phase at epoch 16.

(a)

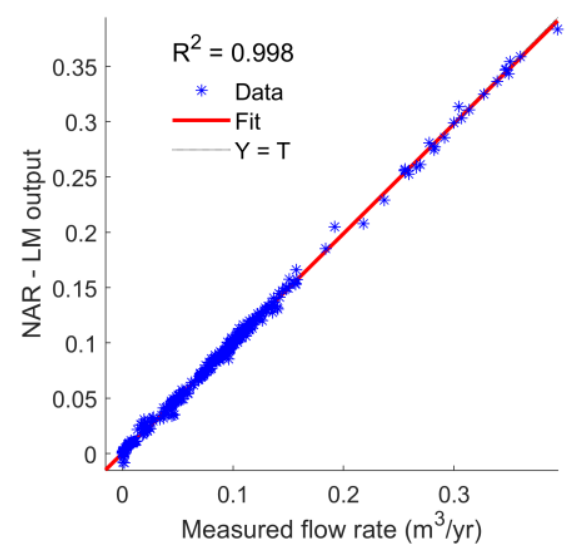

(b)

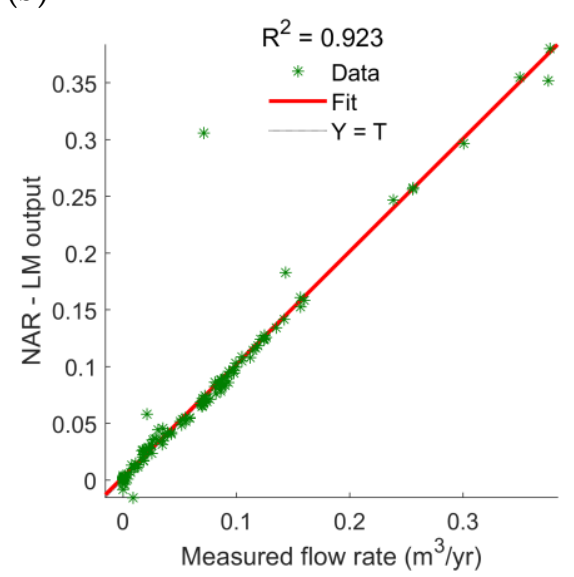

Figure 5-6. Performance of the NAR-LM model for the prediction of fracturing fluid flow rate to the aquifer. (a) Training and (b) Testing.

(a)

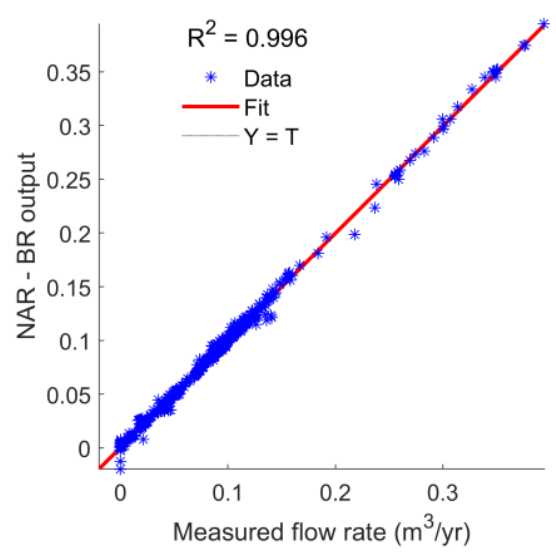

(b)

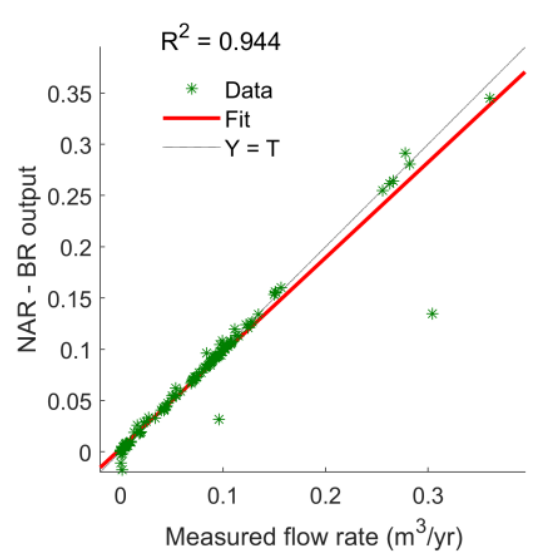

Figure 5-7. Performance of the NAR-BR model for the prediction of fracturing fluid flow rate to the aquifer. (a) Training and (b) testing. 
(a)

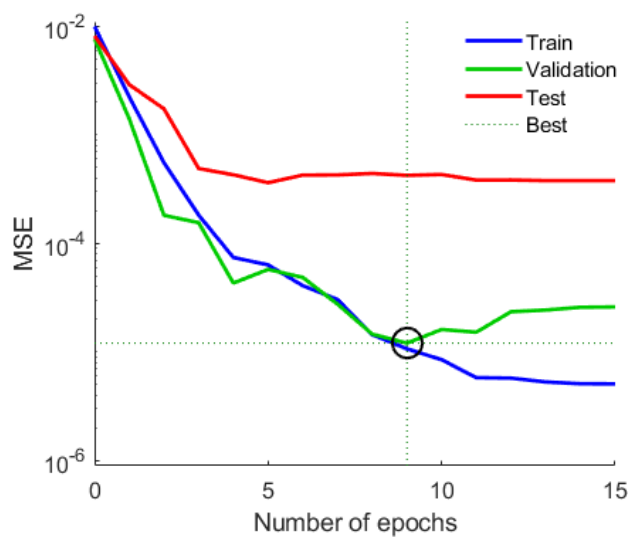

(b)

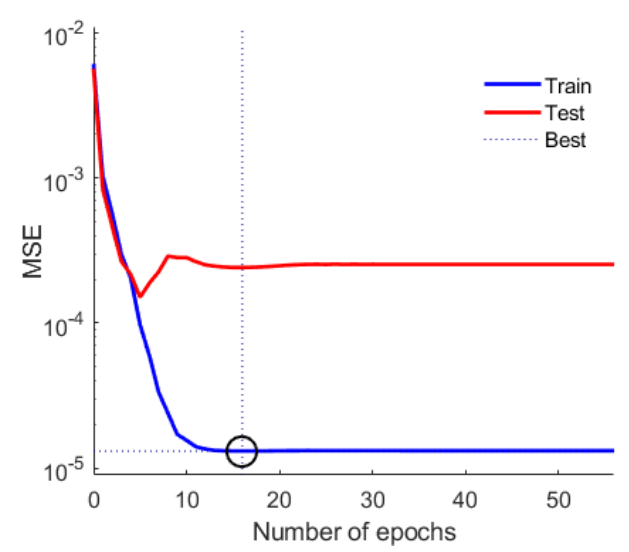

Figure 5-8. Convergence plots of the NAR models in terms of number of epochs. (a) NAR-LM and (b) NAR-BR.

Figure 5-9 illustrates the visualized comparison results of fracturing fluid flow rate predictions for training, validation and testing phases. Note that the response line in this figure is the fracturing fluid flow rate to the aquifer using the base-case values presented in Table 5-1. It was observed that the predicted fluid flow rates to the aquifer followed the measured pattern. The differences between predicted and measured values are small $\left( \pm 7 \times 10^{-3} \mathrm{~m}^{3} /\right.$ year $)$ for the entire time period, indicating that the NAR-LM and NAR-BR models were able to efficiently predict fracturing fluid flow rate to a shallow aquifer over the simulation period. Comparing the response lines and error plots of this figure further indicated the superior performance of the NAR-BR model.

The arrival time of fracturing fluid to shallow groundwater highly depends on the geological and hydrogeological characteristics of the formations between the aquifer and hydrocarbon reservoirs. Evaluating the frequency and impact of fracturing fluid leaks requires extended periods of water-quality monitoring, particularly in cases where overburden layers are not highly fractured and preferential flow paths (e.g. faults and leaky abandoned wells) are not in the vicinity of the operation. Efforts are underway to identify the relationship between oil and gas operations and groundwater contamination in many regions. NAR models could be used for quick and inexpensive but effective assessment of the potential impacts of hydraulic fracturing in the absence of adequate field or modelling data. The results demonstrate that the NAR network trained with LM and BR algorithms could efficiently identify time series patterns in existing fracturing fluid flow rate data to accurately predict the unseen values. 
(a)
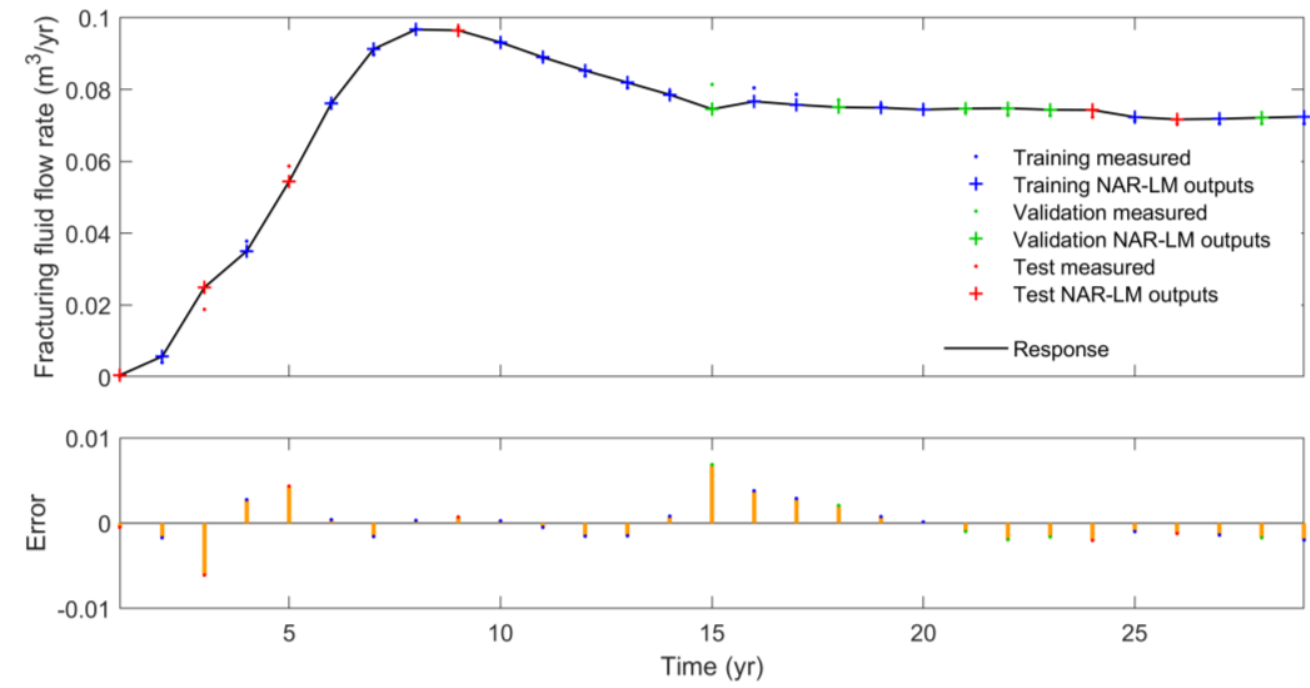

(b)
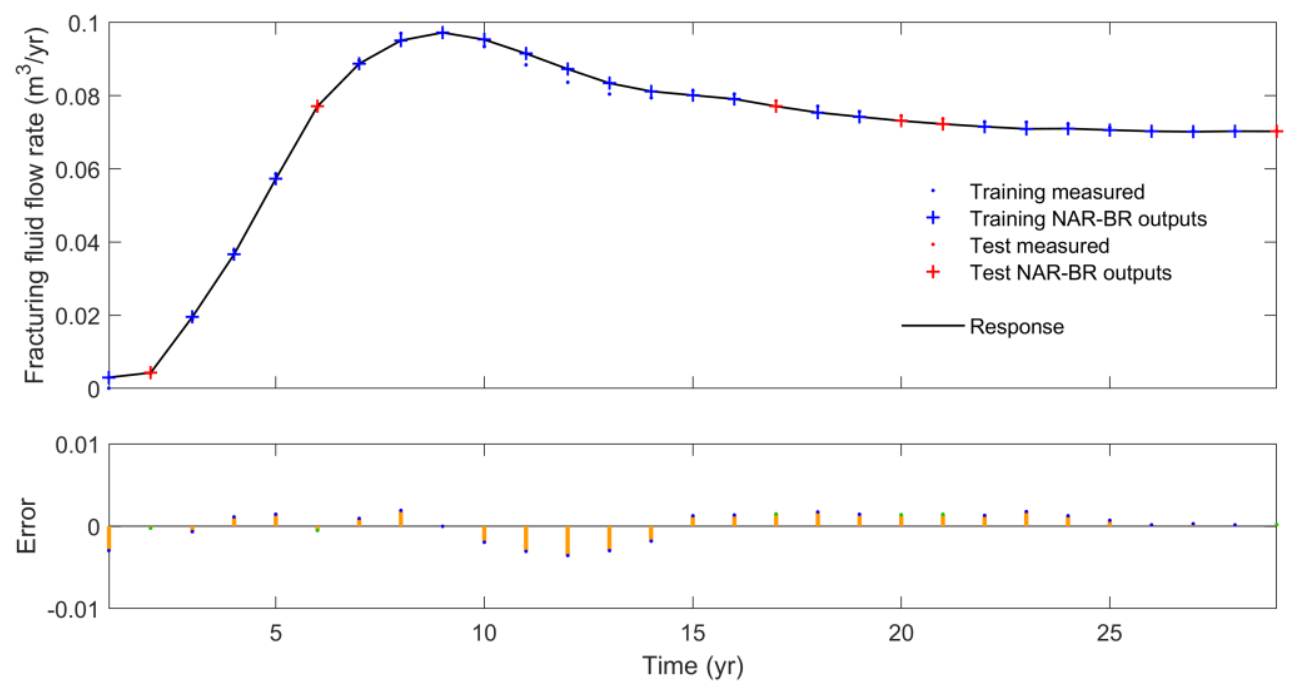

Figure 5-9. Response of (a) NAR-LM and (b) NAR-BR models in predicting fracturing fluid flow rate to the shallow aquifer. The top panels show modeled fluid flow rates of the base-case values in Table 5-1. The bottom panels display the model's performance error in training, validation and testing phases.

Interdisciplinary research has become necessary to break new grounds in science of fluid migration from hydrocarbon formations to groundwater. Our results further highlight the need for strengthening the interdisciplinary collaborations to link petrophysical, geomechanical, and hydrogeological information to develop data-driven models to better evaluate the impact of contaminant leakage to groundwater systems, which may occur by vertical flow migration along pre-existing permeable pathways. Data-driven models have a high potential to recognize the contamination problems in particular when dealing with high data uncertainty, missing and sparse information. For instance, data-driven models can be helpful for assessing regional trends of incidents of groundwater contamination, as the 
availability of consistent data is limited at regional scale. Additional research is essential to build the confidence in the applicability of the methodology and assessing the risks of hydraulic fracturing.

\subsection{Conclusions}

We presented a novel application of a NAR neural network to predict fracturing fluid flow rate into a shallow aquifer following hydraulic fracturing. The dataset used for the network development was taken from Taherdangkoo et al. (2019) and included 744 records of fracturing fluid flow rate into the aquifer, resulting from the upward migration along an abandoned well. Based on the literature review on the performance of training algorithms, the Levenberg-Marquardt and Bayesian Regularization algorithms were selected to train the NAR model. The network structure with three nodes in the hidden layer and two time delays provided the optimal performance.

The results indicated the high accuracy and reliability of NAR-LM and NAR-BR models in the prediction of fracturing fluid flow rate to the aquifer, particularly when using a limited dataset. Both NAR models provides excellent results in terms of goodness-of-fit and independence test. The advantage and power of the proposed models are the fast, cheap and effective approach of calculation in comparison with traditional modelling approaches. By comparing the modelling results, we demonstrated that the predictive performance of the NAR-BR model is slightly better than the NAR-LM model.

There are a few incidents where fracturing fluid and methane were reported to contaminate the aquifer. The NAR models can serve as an additional tool to analyze the incidents of groundwater contamination, especially regional trends of occurrence. In summary, the NAR models trained with LM and BR algorithms showed to have considerable potential in assisting the development of water-quality monitoring programs in the oil and gas regions. 


\section{Chapter 6}

\section{Methane migration through overburden sediments}

This chapter is based on the following paper:

Modelling of methane migration from gas wellbores into shallow groundwater at basin scale Reza Taherdangkoo*1, Alexandru Tatomir ${ }^{1,2}$, Martin Sauter $^{1}$

${ }^{*}$ Corresponding author

${ }^{1}$ Department of Applied Geology, Geosciences Center, University of Goettingen, Goldschmidtstr. 3, D-37077 Göttingen, Germany

${ }^{2}$ Department of Earth Sciences, Uppsala University, Villavägen 16, S-75236 Uppsala, Sweden

Citation: Taherdangkoo, R., Tatomir, A. and Sauter, M., 2020. Modelling of methane migration from gas wellbores into shallow groundwater at basin scale. Environmental Earth Sciences. Under review 


\begin{abstract}
This chapter studies the potential contamination of groundwater via methane migration from a leaky natural gas well through overburden rocks. A two-dimensional, two-phase, twocomponent numerical model is employed to simulate methane and brine upward migration toward shallow groundwater in a generic sedimentary basin. A sensitivity analysis is conducted to examine the influence of methane solubility, capillary pressure - saturation relationship parameters and residual water saturation of overburden rocks, gas leakage rate from the well, tilted formations and low-permeability sediments (i.e. claystones) on the transport of fluids. Results show as expected that the presence of lithological barriers is the most important factor in determining the spatial distribution and volume of methane reaching groundwater. Simulations reveal that methane leakage rate, inclined features, and groundwater flow can greatly affect the transport of methane to various distances from the source of leakage.
\end{abstract}




\subsection{Introduction}

Natural gas extraction from unconventional resources is considered as a promising future source for energy supply as a bridge fuel toward a low-carbon energy system (Brown et al., 2009; McGlade et al., 2013). The surge for unconventional gas development has been projected to have impacts on drinking water resources (Osborn et al., 2011; Sauter et al., 2012; Vengosh et al., 2014). The most frequent and severest threats are likely associated with (1) water acquisition in dry areas, (2) leaks and spills of fracturing fluids and wastewater (i.e. flowback and produced water) at the surface, (3) discharge of inadequately treated wastewater to the environment and (4) migration of liquids (i.e. fracturing fluids and brine) and gas from the deep hydrocarbon reservoir into shallow aquifers (Kissinger et al., 2013; Melchers, 2009; Rice et al., 2018a; Rudolph et al., 2010; Sauter et al., 2012; Schout et al., 2020; U.S. EPA, 2015). Of the potential mechanisms identified, our focus is on the numerical modelling of fluid - fugitive gas and brine - migration from a leaky natural gas well through overburden rocks towards shallow groundwater.

Natural gas is mainly composed of methane with a small amount of carbon dioxide, oxygen, nitrogen and hydrogen sulfide. Methane in stream flows and groundwater is from biogenic and thermogenic processes. The origin of methane can be typically assessed by geological, hydrological and geochemical analysis (Darrah et al., 2014; Jackson et al., 2013; Siegel et al., 2015). Biogenic methane originates from bacterial processes, while the presence of thermogenic methane in groundwater is associated with the upward migration of gas from hydrocarbon reservoirs either over geological time or through anthropogenic activities (Darrah et al., 2015; Gorody, 2012; Osborn et al., 2011; Warner et al., 2012). Although methane is a non-toxic substance, elevated levels of dissolved methane concentrations could possibly affect groundwater quality (Duncan, 2015; Hendry et al., 2017; Lange et al., 2013; Osborn et al., 2011; Rice et al., 2018a). Potential challenges associated with methane contamination of groundwater highlight the need for detailed and publicly available groundwater quality databases, and the extended gas monitoring programs.

Methane can escape from production or intermediate zones through active and abandoned oil and gas wells, fault zones, fracture systems and permeable rocks (Lange et al., 2013; Osborn et al., 2011; Rice et al., 2018b; Steelman et al., 2017; Tatomir et al., 2018; Vengosh et al., 2014). The free-phase methane migrates upward to shallow groundwater by the strong buoyancy force 
resulting from density contrasts between gas and brine. A number of studies have sought to quantify the relative importance of mechanisms and main factors associated with methane migration. Kissinger et al. (2013) was first to develop numerical models to explore methane migration associated with unconventional gas development. They studied several flow paths of leakage fluids to shallow groundwater in the Münsterland Cretaceous basin and the Lower Saxony Basin in Germany. Their results indicate that vertical migration of free-phase methane and a small amount of liquid-phase fluids can occur where there is a connective permeable pathway. Following Kissinger et al. (2013), several authors developed multiphase flow models to study the role of permeable pathways for gas migration to groundwater. For instance, Reagan et al. (2015) performed simulations to investigate flow and transport of methane and brine from the deep gas reservoir to the aquifer. They concluded that the transport along wellbores convey more methane to aquifers than transport along faults and fractures, which is caused by the lower pore volume of the connecting features the well.

Nowamooz et al. (2015) developed numerical models to investigate brine and methane leakage along a decommissioned wellbore. Their results indicated that cementation quality and hydrodynamic properties of the well annulus are the most important factors influencing methane arrival time to the aquifer. Rice et al. (2018b) examined the influence of multiphase parameters on the upward migration of methane from a leaky gas well into groundwater. They found that parameters controlling multiphase flow, i.e., parameters affecting capillarity and relative permeability, exert substantial influence on the volume of methane reaching the shallow groundwater. Moortgat et al. (2018) modeled methane transport from leaky natural gas wells to shallow groundwater. They suggested that pulses of high-pressure leakage into fractured media are required for methane to appear in groundwater at long-distances and at short-term. Klazinga et al. (2019) conducted a series of simulations to explore the role of different factors and parameters on the migration of free-phase methane in an unconfined sandy aquifer. They concluded that subtle permeability and capillary pressure contrasts control the lateral spreading of free-phase methane in the aquifer and therefore leakage into the shallow aquifer system.

The quantification of the size and shape of methane plume in the subsurface still remains a challenge even in simple geologic settings (Cahill et al., 2017; Kissinger et al., 2013; Moortgat et al., 2018; Rice et al., 2018a; Steelman et al., 2017). This is due to the complex nature of 
multiphase, multicomponent flow, and the transport phenomena involved in methane migration. The focus of previous research was mostly on the rapid migration of free-phase methane to groundwater through wellbores, faults and fractures while less effort was directed at understanding the slow migration through overburden rocks as well as the influence of methane solubility on transport. This research is designed to fill the knowledge gap in understanding the upward migration of free- and dissolved-phase methane in the subsurface via conducting sensitivity analysis.

We start with a generic conceptual model derived from an FEP knowledge database and the expert ranking of the key features, events and processes that generate failure scenarios at basin scale (Tatomir et al., 2018, 2015). From the conceptual model, we build two-dimensional twophase, two-component numerical models to study the flow and transport of methane and brine from a leaky gas well into shallow groundwater aquifer through overburden sediments for a period of 25 years. First, the impact of methane solubility on fluid migration dynamics and the leakage rates into the aquifer is explored. Then, we examine the influence of (1) the parameters describing capillarity pressure- and relative permeability-saturation of shallow and overburden rocks, (2) the low-permeability sediments, (3) the tilted formations (ranges between $0^{\circ}$ to $3^{\circ}$ ) and (4) the methane leakage rate from the gas wellbore. Finally, the findings are compared with results from observational studies to better understand real-world scenarios.

\subsection{Methods and materials}

\subsubsection{Conceptual model for methane and brine migration at the basin scale}

We build a generic basin-scale two-dimensional numerical model, Figure 6-1, comprising three horizontal layers. A thin $(21 \mathrm{~m})$ continuous low permeability unit, e.g. claystone, is assumed at shallow depth (171 m) overlain by coarse sediments (Taherdangkoo et al., 2018). The model spans across a domain of $10 \mathrm{~km}$ length and $1.8 \mathrm{~km}$ depth. A large section of the top boundary, i.e. $95 \%$ of the model length, is defined as a constant recharge boundary intersecting a constant hydraulic head boundary ( $5 \%$ of the model length). The groundwater recharge rate amounts to $0.05 \mathrm{~m} \mathrm{yr}^{-1}$. The lateral sides of the domain are set to no-flow boundaries. It should be noted that the generic conceptual model is built based on the median values reported for 
sedimentary basins in North America and Europe. For instance, according to the dataset, the median values for claystone thickness and depth are 21 and $171 \mathrm{~m}$, respectively (Doornenbal and Stevenson, 2010; Gleeson et al., 2016; Lehner et al., 2008; Peters et al., 2018; Taherdangkoo et al., 2018).

The conceptual model assumes fracture propagation into overlying formations during a hydraulic fracturing treatment (Kissinger et al., 2013; Lange et al., 2013; Moortgat et al., 2018; Reagan et al., 2015; Tatomir et al., 2018). It is common in the oil and gas industry to temporarily shut-in a well because of operational and economic reasons. The pressure in the gas reservoir does not decay during the shut-in period. Under this condition, should the well annulus intersect with a fracture/fracture system and thus connect the gas reservoir with the overburden, considerable leakage can be expected (Gassiat et al., 2013; Moortgat et al., 2018; Reagan et al., 2015; Tatomir et al., 2018). The model assumes that during shut-in gas leaks into the overburden at high flow rates, followed by a much lower rate once the production is resumed.

The leakage rate depends on various factors such as reservoir pressure, reservoir gas saturation, in-situ and relative permeability (Nowamooz et al., 2015; Reagan et al., 2015). Kissinger et al. (2013) modeled constant leakage of methane from the reservoir into the overburden for 100 years. The authors emphasized that the constant escape of gas is regarded as "a conservative" assumption. Reagan et al. (2015) suggested that incidents of gas leakage from the reservoir are likely to be limited in time. Moortgat et al. (2018) considered a high methane leakage rate during the shut-in period, followed by a lower rate during the production. We applied leakage rates published in Moortgat et al. (2018) because of its similarity to our conceptual model. However, the geological setting, rock types and tectonics are different in this study.

The leakage is assumed to occur at the top of the gas reservoir through the fracture system intersecting the overburden, thus instead of explicitly considering the reservoir in the model setup, it is included as a boundary condition. The gas entering the domain is assumed to be pure methane and the presence of other thermogenic gases is neglected (Nowamooz et al., 2015; Rice et al., 2018a; Roy et al., 2016). The leakage source measures $100 \mathrm{~m}$ in length and it is situated at the bottom of the domain at $40 \%$ of the model length $(3950 \leq x \leq 4050)$ for all 
subsequent simulations. The remaining of the lower boundary is set to no-flow condition. The methane leakage rate is estimated based on the production rate from the borehole. A typical production rate of 300 MSCFD (million standard ft $3 /$ day) (Moortgat et al., 2018) (35680 m³/day at reservoir condition) is assumed for the base-case model. Methane leaks into the overburden at a high inflow rate of $8920 \mathrm{~m}^{3} /$ day, i.e. $25 \%$ of the production during the 7 days of the shutin period, followed by an inflow rate of $892 \mathrm{~m}^{3} /$ day, i.e. $10 \%$ of the initial leakage rate, for the next 2 years. The upward migration of methane and brine is monitored for 25 years of simulation time.

The low-permeability unit, i.e. claystone, is a laterally extensive single layer overlying the entire basin which might contain discontinuities. Moreover, multilayered systems where low permeability units are interbedded within permeable sediments may exist. Each field configuration could lead to a different contamination distribution in the subsurface based on its unique stratigraphic, geophysical and hydrodynamic characteristics (Sauter et al., 2012). Herein, we first assume a single continuous claystone across the entire model length to obtain first order information on the influence of low-permeability sediments on methane migration. We then consider different configurations to explore the impact of claystone integrity and multi-layered clay systems.

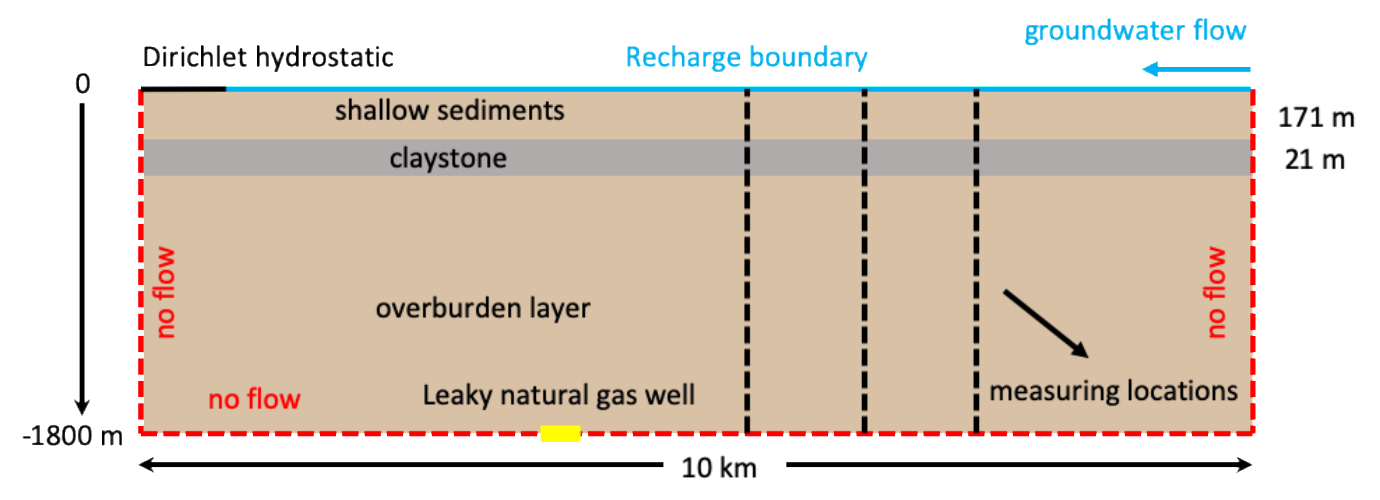

Figure 6-1. Generalized conceptual model of methane and brine migration from the gas reservoir into the overlying formations for the base-case model. The low-permeability layer, i.e. clay, is indicated in gray and permeable sediments in light brown. The dark dashed lines represent the monitoring locations at various distances to the leaky gas well. The leakage area is presented in yellow. The boundary conditions employed in the simulations are shown. 


\subsubsection{Numerical modelling approach}

The dominant physical and chemical processes for fluid migration are advection (viscous, buoyant, and capillary), dissolution and diffusion (Nowamooz et al., 2015). Methane sorption is assumed to be negligible (Rice et al., 2018b) and will not be discussed further. The flow and transport of fluids are simulated using miscible and immiscible flow models. The miscible flow model considers advection, diffusion and dissolution processes while the immiscible model considers migration via advection and diffusion.

All simulation runs are conducted with the open-source numerical simulator DuMux (Flemisch et al., 2011). For spatial discretization, the box method, a vertex (or node)- centered finite volume method based on a finite element grid, is used. A fully implicit time discretization scheme using the Newton method is applied to solve the system of non-linear partial differential equations (Helmig, 1997). The grid resolution equals 500 cells in the $x-$ direction and 180 cells in the y-direction. We use 2P2C (two-phase, two component) and 2P (two-phase) modules for simulating isothermal miscible and immiscible two-phase flow and transport of fluids in porous media, respectively. The binary diffusion and the diffusive mass fluxes in each phase are computed according to Fick's law. The mass balance equation for a two-phase two-component model can be formulated for each $k$ as follows (Dietrich et al., 2005; Helmig, 1997):

$$
\begin{aligned}
& \frac{\partial\left(\varnothing \sum_{\alpha} \rho_{\alpha} X_{\alpha}^{k} S_{\alpha}\right)}{\partial t}-\sum_{\alpha} \nabla \cdot\left\{\rho_{\alpha} X_{\alpha}^{k} \frac{k_{r \alpha}}{\mu_{\alpha}} \boldsymbol{K}\left(\operatorname{grad} P_{\alpha}-\rho_{\alpha} \boldsymbol{g}\right)+\rho_{\alpha} D_{p m, \alpha}^{k} \operatorname{grad} X_{\alpha}^{k}\right\}-q^{k}=0 \\
& \alpha \in\{w, n\} \text { and } k \in\{\text { brine, methane }\}
\end{aligned}
$$

where the index $\alpha$ represents the wetting and non-wetting phases. The index $\kappa$ represents the components brine and methane. $\varphi$ is the effective porosity and $\rho_{\alpha}$ is the density of phase $\alpha . X_{\alpha}^{k}$ is the mass fraction of component $\kappa$ in the phase $\alpha, S_{\alpha}$ is the saturation, $\mu_{\alpha}$ is the dynamic viscosity and $k_{r \alpha}$ is the relative permeability. $\boldsymbol{K}$ is the intrinsic permeability tensor, $P_{\alpha}$ is the phase pressure, $\mathbf{g}$ is the gravitational acceleration and $D_{P m, \alpha}^{k}$ is the effective diffusion coefficient of the porous medium.

Methane can exist as a free-phase gas with a limited amount of water vapor or dissolved in the aqueous phase. The amount of water in the gas phase based on Duan et al. (1992a). Methane solubility varies over a wide range of temperatures, pressures and brine compositions (Duan et al., 1992a; Duan and Mao, 2006). Methane dissolution in the aqueous 
phase is assumed to occur at thermodynamic equilibrium and gas phase saturations were simultaneously updated at each time step to consider mass loss to brine. Assuming methane at equilibrium with respect to water, methane solubility $\left(\mathrm{mg} \mathrm{L}^{-1}\right)$ can be computed following the Duan et al. (1992a) approach as in:

$$
\ln \frac{x_{\mathrm{CH}_{4} P}}{m_{C H_{4}}}=\frac{\mu_{C H_{4}}^{l(0)}}{R T}-\ln \emptyset_{C_{4}}+\sum_{c} 2 \lambda_{C_{H_{4}-c}} m_{c}+\sum_{a} 2 \lambda_{C H_{4}-a} m_{a}+\sum_{c} \sum_{a} \zeta_{C_{4}-c-a} m_{c} m_{a}
$$

where $m$ is the molality of $\mathrm{CH}_{4}$ or salts in the liquid phase and $x$ is the composition in the vapor phase. $P$ is the total pressure and $\mathrm{R}$ is the universal gas constant $\mu_{\mathrm{CH}_{4}}^{l(0)}$ is the standard chemical potential of $\mathrm{CH}_{4}$ in the liquid phase and $\varnothing$ is the fugacity coefficient. The index a and c are anion and cation, respectively. $\lambda_{\mathrm{CH}_{4}-\mathrm{ion}}$ and $\zeta_{\mathrm{CH}_{4}-\mathrm{C}-a}$ are interaction parameters. $\frac{\mu_{C H_{4}}^{l(0)}}{R T}$ and the interaction parameters are calculated by Pitzer et al. (1984) method (Duan et al., 1992a).

\subsubsection{Material properties}

The variations in porosity and permeability are typically driven by changes in mineralogical composition, i.e. mineral grains and cement, depositional facies, burial depth and postdepositional processes (Weibel et al., 2012). In our model, the permeability and porosity of each layer are calculated using depth and lithology related equations (Carman, 1937; Kozeny, 1927; Luijendijk and Gleeson, 2015; Sclater and Christie, 1980). The mineral composition of the claystone comprises 50\% kaolinite and 50\% illite (Luijendijk and Gleeson, 2015). The estimated porosity and vertical permeability of claystone are 0.05 and $1.1 \times 10^{-20} \mathrm{~m}^{2}$, respectively (Figure 6-2).

The shallow and overburden sediments contain $90 \%$ sand and $10 \%$ clay to obtain realistic permeability values (Gleeson et al., 2011). Permeability and porosity of these rocks in the $y$ direction vary approximately from $6.8 \times 10^{-14} \mathrm{~m}^{2}$ to $2.6 \times 10^{-15}$ and from 0.3 to 0.12 , respectively as shown in Figure 6-2. The anisotropy ratio $\left(\mathrm{K}_{\mathrm{h}} / \mathrm{K}_{\mathrm{v}}\right)$ is set to 100 in the claystone and 10 in the surrounding sediments. The temperature at the top of the domain is $10{ }^{\circ} \mathrm{C}$, with a vertical temperature gradient of $0.03{ }^{\circ} \mathrm{C} \mathrm{m}^{-1}$. The domain is initially water saturated. The residual water saturation is set to 20\% (Nowamooz et al., 2015; Rice et al., 2018b) and the residual gas saturation is $0.01 \%$ (Rice et al., $2018 b$ ).

Methane is the predominant component in the gas mixture and small amounts of other components (e.g. $\mathrm{N}_{2}, \mathrm{CO}_{2}, \mathrm{C}_{2}$, and $\mathrm{C}_{3}$ ) has a negligible influence on the density and viscosity 
of the gas phase (Nowamooz et al., 2015). For simplification, the model considers the leaked gas as pure methane. Brine is modeled as a pseudocomponent and represents pure water with a given salinity. Brine density and viscosity are calculated following Batzle and Wang (1992) equations. Methane density and viscosity are calculated based on Duan et al. (1992b) and Daubert (1989), respectively. The diffusion coefficient of water in the gas phase is calculated based on Fuller et al. (1966). Since the empirical equations for estimating the diffusion coefficient in infinite solution presented in Reid et al. (1987) all show a linear dependency on temperature, we simply scale the experimentally obtained diffusion coefficient of the dissolved methane in the water phase given in Kobayashi (2004) by temperature. Kobayashi (2004) suggested the diffusion coefficient of $3.55 \times 10^{-9} \mathrm{~m}^{2} / \mathrm{s}$.

As methane leakage rates reported by Moortgat et al. (2018) are specified for surface conditions, a gas formation volume factor $\left(\mathrm{Bg}_{\mathrm{g}}\right)$ of $0.0042 \mathrm{ft}^{3} \mathrm{SCF}^{-1}$ is used to convert the values to reservoir conditions. The $\mathrm{B}_{\mathrm{g}}$ is calculated using the averaged values reported by Edwards and Celia (2018). The general model property values are presented in Table 6-1. The capillary pressure and the relative permeability are calculated as a function of saturation using Brooks and Corey (1964) equations as follows:

$$
P_{c}=P_{d} S_{e}^{\frac{-1}{\lambda}}
$$

where $P_{d}$ is the entry pressure of the porous medium and is set to $10 \mathrm{kPa}$ for the overburden and shallow sediments and $12 \mathrm{kPa}$ for the claystone. The Brooks-Corey parameter $(\lambda)$ is set to 2.0 for the entire domain. The relative permeability functions, $K_{r}(S)$, can be calculated by the following equations:

$$
\begin{aligned}
& K_{r w}=S_{e}^{\frac{2+3 \lambda}{\lambda}}, \text { for the wetting phase } \\
& K_{r g}=\left(1-S_{e}\right)^{2}\left(1-S_{e}^{\frac{2+\lambda}{\lambda}}\right), \text { for the non-wetting phase }
\end{aligned}
$$

The effective saturation $S_{e}$ is defined using the following equation. $S_{w r}$ and $S_{g r}$ are irreducible saturation of liquid and gas phases, respectively.

$$
S_{e}=\frac{S_{w}-S_{w r}}{1-S_{g r}-S_{w r}}
$$


Table 6-1. List of parameter values for the reference setting.

\begin{tabular}{|l|l|l|}
\hline Parameter & Unit & Value \\
\hline Compressibility of solid phase & $\mathrm{Pa}^{-1}$ & $4.5 \times 10^{-10}$ \\
\hline Recharge at the top boundary & $\mathrm{m} \mathrm{year}^{-1}$ & 0.05 \\
\hline Temperature at the top boundary & ${ }^{\circ} \mathrm{C}$ & 10 \\
\hline Temperature gradient & ${ }^{\circ} \mathrm{C} \mathrm{m}^{-1}$ & 0.03 \\
\hline Gas formation volume factor, $\mathrm{Bg}_{\mathrm{g}}$ & $\mathrm{ft}^{3} \mathrm{SCF}^{-1}$ & 0.0042 \\
\hline Methane density at atmospheric condition, $\mathrm{Og}$ & $\mathrm{kg} \mathrm{m}^{-3}$ & 0.72 \\
\hline Entry pressure for the clay, $\mathrm{Pd}$ & $\mathrm{Pa}$ & 12000 \\
\hline Entry pressure for the overburden and shallow sediments, $\mathrm{Pd}_{\mathrm{d}}$ & $\mathrm{Pa}$ & 10000 \\
\hline Brooks-Corey parameter, $\lambda$ & & 2.0 \\
\hline residual water saturation, $\mathrm{S}_{\mathrm{wr}}$ & & 0.2 \\
\hline residual gas saturation, $\mathrm{S}_{\mathrm{gr}}$ & & 0.0001 \\
\hline
\end{tabular}

(a)

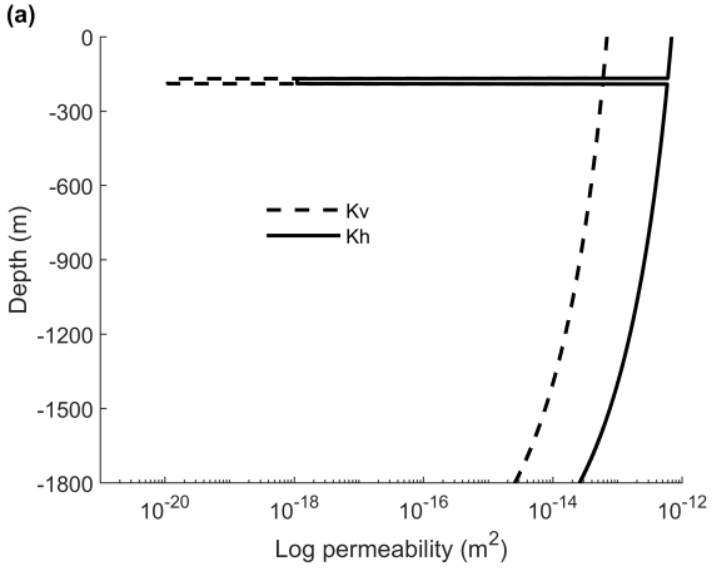

(b)

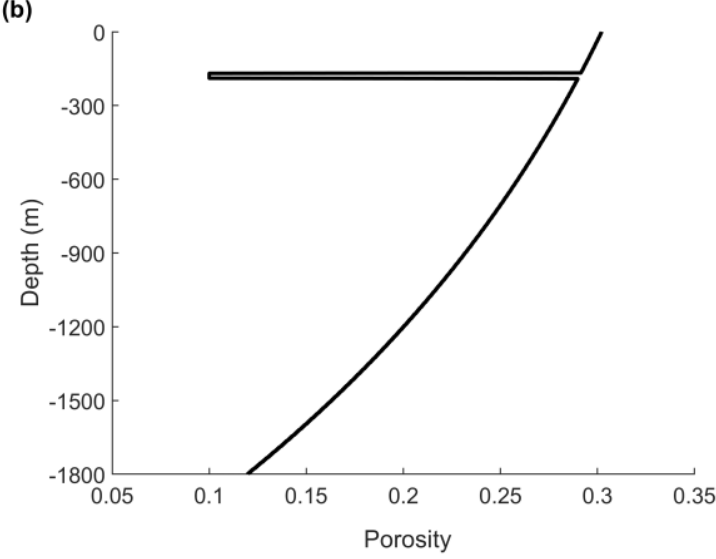

Figure 6-2. The distribution of (a) permeability and (b) porosity in the domain.

\subsubsection{Simulation strategy}

The base-case models explore miscible and immiscible flow and transport of fluids to shallow layers for a period of 25 years. The temporal and spatial behavior of the plume in the subsurface and the change in volumetric flow rates to shallow groundwater of methane are observed. Moreover, methane and brine flow rates at various distances from the leaky gas well $(\Delta \mathrm{L}=2000,3000$ and $4000 \mathrm{~m})$ are monitored. These locations can be visualized as intersections of the aquifer with conductive permeable pathways such as faults and fractures, i.e. preferential flow paths for the transport of fluids in the upper crust. This choice allowed us to remarkably improve the tracking of the methane plume at various distances from the gas wellbore in basins with or without vertical permeable pathways.

A series of sensitivity analyses is designed to examine the roles of different geometric configurations and parameter combinations on the migration of fluids. Parameter selection is 
based on a comprehensive features, events, and processes (FEPs) procedure highlighting the relevant importance of the key factors influencing the upward movement of contaminants (Tatomir et al., 2018). Firstly, we explore the sensitivity of hydrodynamic properties $\left(P_{d}, \lambda\right.$, and $\left.\mathrm{S}_{\mathrm{wr}}\right)$ of shallow and overburden sediments. The displacement or entry pressure $\left(P_{d}\right)$ of the porous medium changes between 2 and $20 \mathrm{kPa}$ and is set to $10 \mathrm{kPa}$ in the base-case model. A large pore size distribution index $(\lambda)$ indicates a comparatively uniform pore size distribution, while small values of $\lambda$ describe a non-uniform distribution (Brooks and Corey, 1964). $\lambda$ varies from 2 to 6 and is set to 2 in the base-case model. These values are chosen to cover a wide range of values of pore size distribution index and entry pressure. The value of residual water saturation varies between 0.15 and 0.25 and equals to 2.0 in the base-case model.

We include simulations for sedimentary basins with dip angles of $0^{\circ}, 1^{\circ}$ and $3^{\circ}$. We rotate the model coordinate system to tilt the geological geometric configuration. We keep the top of the domain at the same depth and increase the maximum depth by increasing the dip. Then, the impact of methane leakage rate from the wellbore is explored. As an alternative to the high leakage with short duration, two low constant gas inflow rates of 60 and $120 \mathrm{~m}^{3} / \mathrm{day}$ for uninterrupted 25 years are considered.

The sensitivity analysis then considers a discontinuity, 10\% of the model length, in the middle of the claystone to examine the role of low-permeability rocks on fluid flow and mass transport. Further simulations include the integration of a multi-layered claystone system with interbedded permeable rocks at shallow depth. We assume two different filed settings by varying the geometry of the clay system, which consists of four clays with random distribution, integrity and depth. All clays are embedded between 150 and $350 \mathrm{~m}$ depth and have the same thickness of the base-case value, $21 \mathrm{~m}$. This sensitivity analysis is presented to examine how the migration of methane from a deep gas reservoir is impacted by sequences of low-permeability rocks.

\subsection{Results and discussion}

\subsubsection{Base-case model}

The gas saturation and pressure profiles of methane leakage using the miscible flow model are shown in Figure 6-3 and Figure 6-4, respectively. In this model, methane exists as free and 
dissolved phase gas in the aqueous phase. Methane migrates upward predominantly by buoyancy and reaches the claystone after approximately 695 days. The strong vertical buoyancy controls the direction of methane migration; thus the lateral spread is limited. The tube-shaped flow of gas is partially due to the consideration of a homogenous model domain. The diameter of the plume around the leakage source is approximately $320 \mathrm{~m}$ after 2 years and remains constant. The claystone overlying the overburden sediments constitutes an effective barrier to the upward movement of fluids. For this configuration, horizontal viscous flow forces overcome buoyancy forces and methane spreads laterally along the horizontally stratified layer. As illustrated Figure 6-5, an important volume of gas phase accumulates at the claystone base after 25 years reaching eventually the aquifer through connective pathways, shown by dashed white lines. Permeable pathways, such as fractures, faults and abandoned wells through barrier rocks play an important role for fluid migration in the upper crust. In the context of hydraulic fracturing operations, the presence of these flow paths in the proximity of a leaky gas well could lead to the early occurrence of methane in groundwater (Cahill et al., 2017; Darrah et al., 2014; Moortgat et al., 2018).

(a)

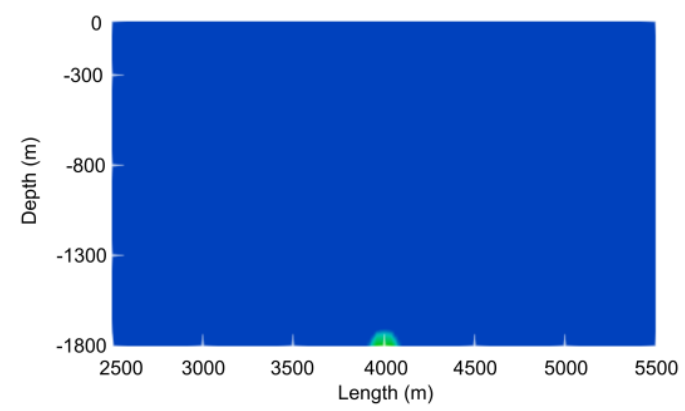

(b)

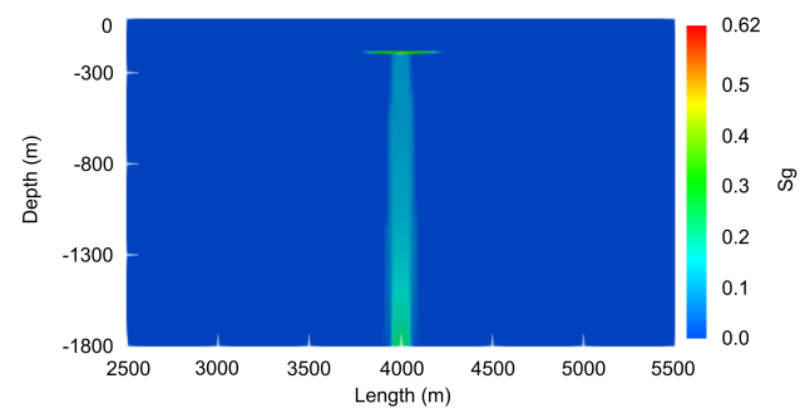

Figure 6-3. Gas phase saturation profiles after (a) 7 days and (b) 2 years of methane leakage into the overburden formations. This model assumes miscible flow and transport of fluids.

(a)

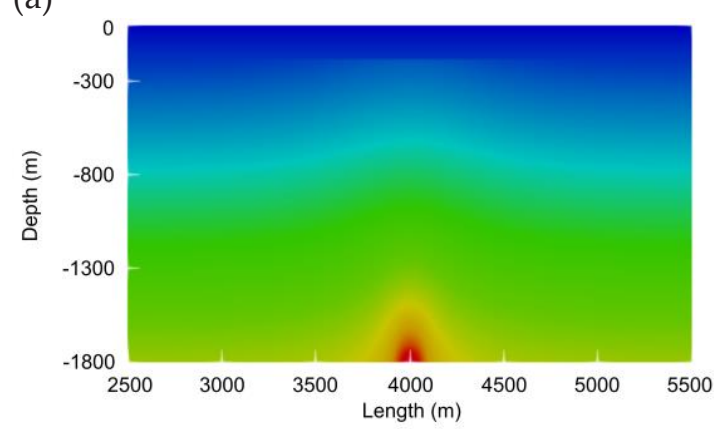

(b)

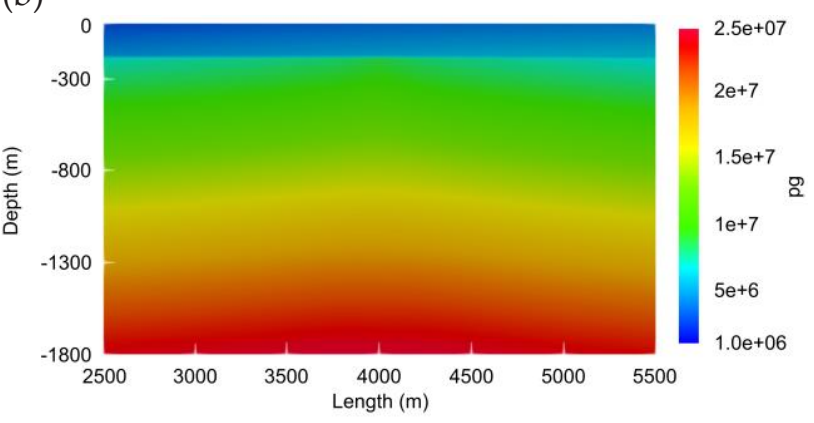

Figure 6-4. Pressure profiles after (a) 7 days and (b) 2 years of methane leakage into the overburden formations. This model assumes miscible flow and transport of fluids. 
For the time 25 years after the start of leakage, the dissolved methane $\left(\mathrm{mg} \mathrm{L}^{-1}\right)$ in the aqueous phase is presented in Figure 6-5. The density of the gas phase and the composition of the aqueous phase strongly depend on pressure and temperature. Methane solubility in the aqueous phase is higher in deep formations due to the increase in pressure, thus more methane is dissolved in brine before the appearance of the gas phase (Duan et al., 1992a; Duan and Mao, 2006). Following the commencement of leakage, free-phase methane moves upward by buoyancy and progressively dissolves in brine. The dissolved methane is transported by groundwater flow by advection and hydrodynamic dispersion processes. The high buoyancy force from density contrasts controls the long-term upward migration of free-phase methane. As illustrated by the pressure profiles (Figure 6-4), pressure decreases with depth resulting in (i) a significant decrease in the density of the gas phase and (ii) release of some of the dissolved methane to the gas phase. Accordingly, more highly buoyant free-phase methane appears at shallow depths with the consequence of increasing the upward flow rate. For such conditions, large amounts of free-phase methane migrate to the groundwater system within a relatively short time.

(a)

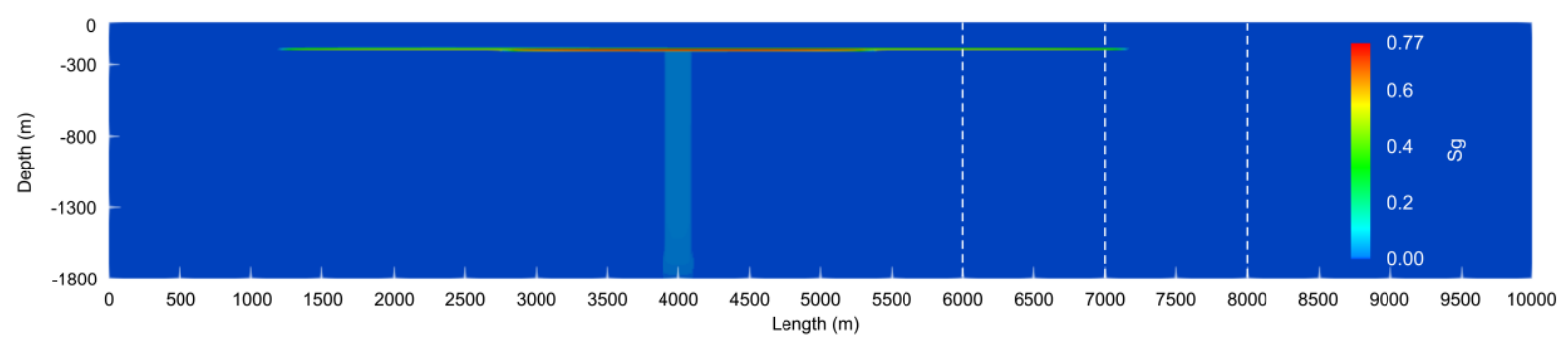

(b)

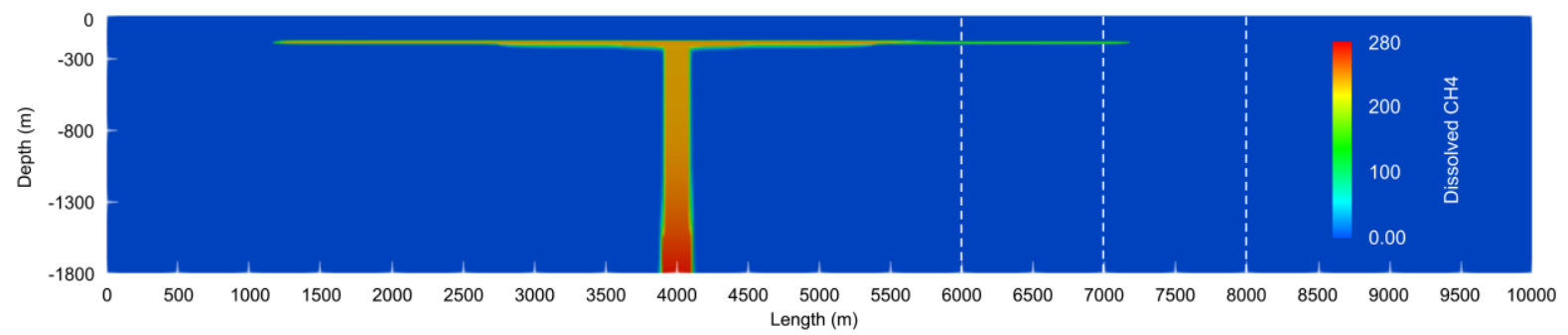

Figure 6-5. Spatial distribution of (a) gas phase saturation and (b) dissolved methane (mg L-1) in the aqueous phase after 25 years of methane leakage into the overburden formations. The white dashed lines show the monitoring locations. 
The gas phase saturation and pressure profiles of the immiscible flow model are shown in Figure 6-6 and Figure 6-7, respectively. Methane reaches the base of the claystone after approximately 660 days. The methane accumulation at the claystone is followed by the extensive horizontal spreading. Similar to the methane migration in the miscible flow model set-up, the diameter of the plume around the leakage source measures approximately $320 \mathrm{~m}$ after 2 years and remains unchanged. In both miscible and immiscible flow models, the lateral spread of the plume before reaching the claystone is limited, partially due to the assumption of a homogenous domain.

The mass flow rates of methane and brine are obtained from the total non-wetting phase and wetting phase flow rates calculated from advection, diffusion and dissolution processes. Figure 6-8a and Figure 6-8b represent methane and brine volumetric flow rates at 2000, 3000, and $4000 \mathrm{~m}$ distances to the gas well, respectively. Methane reaches the monitoring locations during the extensive lateral spreading at the claystone base. Methane flow rate increases rapidly to its maximum value and then shows a continuous decrease with time (Figure 6-8). Mass flow rates of methane and brine depend on the distance from the well visible in the decreasing peak values with an increase in distance. The flow rates of fluids corresponding to the miscible model are slightly lower compared to the immiscible model, indicating the effect of gas dissolution on transport.

The pressure gradient created by the gas influx causes brine to slowly migrate in the subsurface (Figure 6-8). Brine flow rate at the measuring locations increases during the leakage and declines sharply after the drop in pressure. The first peak value corresponds to the end of the shut in and the second peak corresponds to the end of leakage. Brine flow changes in the direction of regional groundwater flow after approximately 5 years due to the combined influence of regional hydraulic gradient, cease of gas leakage and negative buoyancy. Although brine flows towards the monitoring locations (i.e. highly permeable pathways), the probability of deep brine reaching shallow groundwater is low (Gassiat et al., 2013; Osborn et al., 2011; Pfunt et al., 2016; Taherdangkoo et al., 2019).

As illustrated, using miscible and immiscible flow models result in slightly different methane plume sizes and travel times to groundwater. This is due to the low solubility of methane in 
the ambient water, particularly at shallow depths. The influence of methane solubility on transport becomes less significant once the plume spreads at shallow depths.

(a)

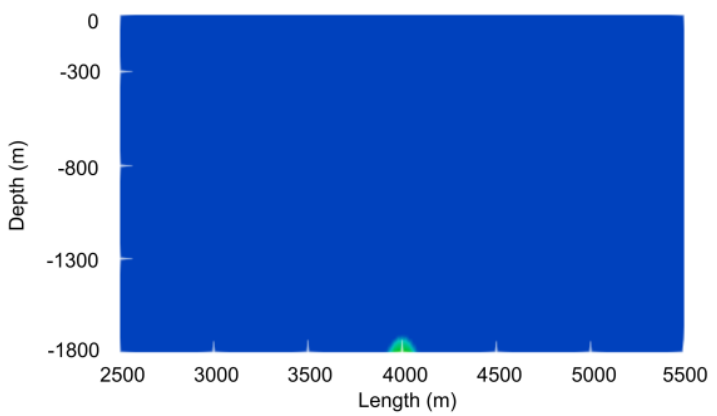

(b)

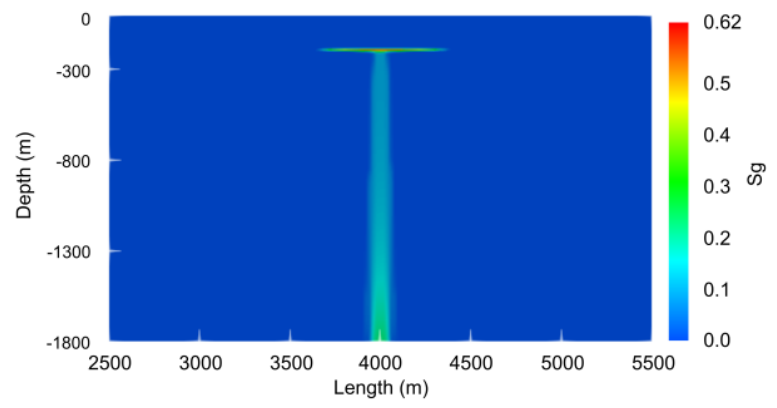

Figure 6-6. Gas phase saturation profiles after (a) 7 days and (b) 2 years of methane leakage into the overburden formations. This model assumes immiscible flow and transport of fluids.

(a)

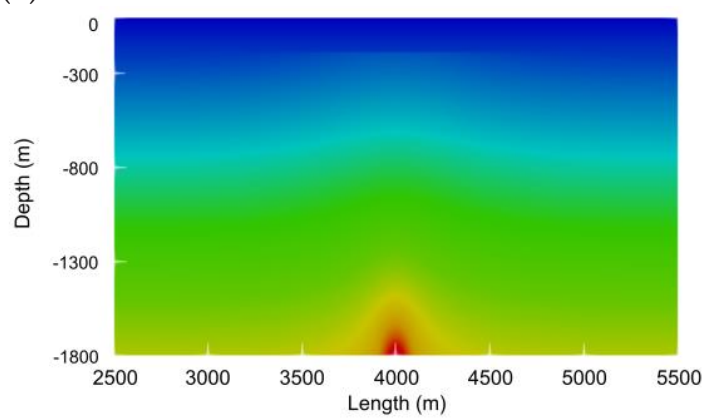

(b)

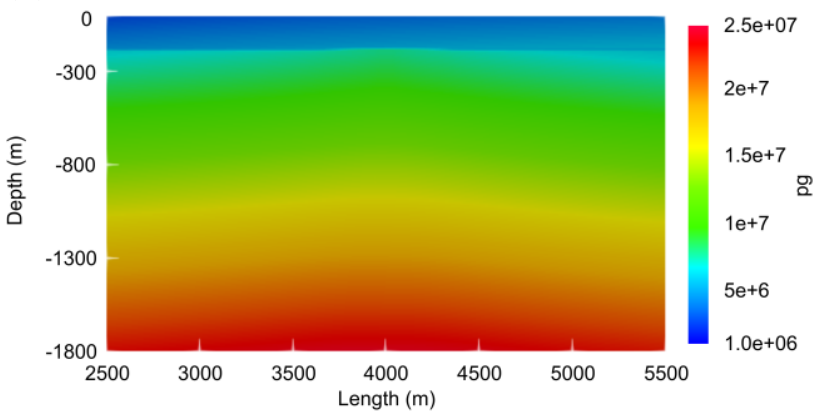

Figure 6-7. Pressure profiles after (a) 7 days and (b) 2 years of methane leakage into the overburden formations. This model assumes immiscible flow and transport of fluids.
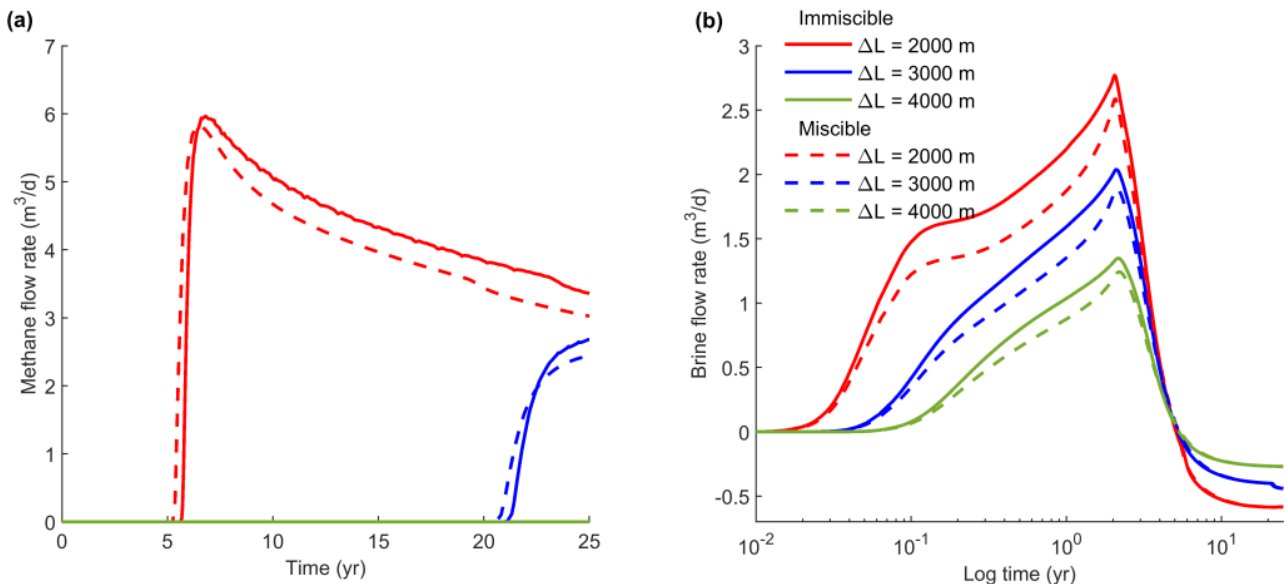

Figure 6-8. (a) Methane and (b) brine flow rates at 2000, 3000, $4000 \mathrm{~m}$ distances from the leaky gas well for immiscible and miscible flow models. 


\subsubsection{Sensitivity analysis}

The sensitivity analysis is conducted employing the miscible flow model. Firstly, the influence of key parameters including Brooks-Corey parameters, residual water saturation, tilted geometries and methane leakage rates are examined. Then, the impacts of the claystone integrity and multi-layered claystone systems on the spatial distribution of the methane plume in the subsurface and the arrival time to shallow groundwater are discussed.

\subsubsection{Geological and hydrogeological parameters}

The impacts of hydrodynamic properties (i.e. $S_{w r}, P_{d}, \lambda$ ) of the shallow and overburden sediments on the transport of methane are illustrated in Figure 6-9(a-c). The entry pressure in the capillary pressure function is inversely correlated with methane flow rate at the monitoring locations. A higher entry pressure requires a higher non-wetting phase (i.e. gas) pressure to displace the ambient water resulting in the reduction of methane flow rates, as shown in Figure 6-9a. Varying the entry pressure has a substantial influence on the flow rate, when $\mathrm{P}_{\mathrm{d}}$ is varied over a large range.

The pore size distribution index $(\lambda)$ is a shape parameter in the capillary pressure function and the relative permeability model and affects the phase saturation in porous media. An inverse relationship between $\lambda$ and methane flow rate is observed as an increase in the $\lambda$ value, increasing the degree of saturation of the water phase. Varying $\lambda$ within a small range has negligible impacts on arrival times and flow rates of methane, while the influence of varying $\lambda$ over a wider range is noticeable.

Residual water saturation $\left(\mathrm{S}_{\mathrm{wr}}\right)$ is important with respect to its influence on the relative permeability. A positive correlation between $S_{w r}$ and methane flow rates is observed as an increase in the value of $S_{\mathrm{wr}}$ decreasing the amount of gas phase trapped in the pore spaces. The effects of varying $S_{\mathrm{wr}}$ on breakthrough time and volume of methane reaching monitoring locations are more substantial in our models in contrast to a variation in $\mathrm{P}_{\mathrm{d}}$ and $\lambda$.

In a sedimentary basin with a larger dip angle $\left(\operatorname{dip} \geq 3^{\circ}\right)$, the lateral buoyancy component further contributes to the horizontal spreading of the plume. Methane moves up dip along tilted layers and spreads over a large distance starting from the leaky wellbore. The lateral transport distances of methane are significantly higher even for only minor tilt $\left(1^{\circ}\right)$ as compared to a horizontal domain. In the formation with $3^{\circ}$ tilt in the $x$-direction, methane 
reaches the monitoring location $4000 \mathrm{~m}$ away from the gas wellbore within 7.2 years, but it does not spread that far in the horizontal dimension (Figure 6-9d). Our results suggest that in tilted formations/layers methane can laterally migrate distances of several kilometers from a leaky gas well.

\subsubsection{Methane leakage rate from the wellbore}

We compare the pulse of high leakage rates assumed in the base-case model with continuous slow leakage of gas. The lowest inflow rate, i.e. $60 \mathrm{~m} 3 / \mathrm{d}$, results in a delayed arrival of methane at the monitoring locations in contrast to other cases, clearly showing less spatial spreading. The plume size is, as expected, larger with longer exposure to the leakage. Persistent longterm leakage leads to high methane concentrations in shallow layers, which induces higher contamination risks to groundwater. The results demonstrate that a pulse of high leakage rate is required to produce methane contamination in groundwater within short times. As illustrated in Figure 6-9e, leakage rate and leakage period have significant impacts on the propagation of the plume in the overburden and accumulated methane volume in shallow groundwater.

Varying gas inflow rates in a tilted formation $\left(\sim 1^{\circ}\right)$ (Figure 6-9f) has substantial influence on the temporal and spatial distribution of the plume in the overburden. The persistent leakage in a tilted formation leads to the extensive spreading in the direction of the tilt enhancing the risk to groundwater within a short-time scale, particularly with the presence of permeable pathways within this range. Under these conditions, free- and dissolved-phase methane could affect a larger area and millions of kilograms of methane are most likely to be transported to the aquifer. This scenario indicates that gas leakage in a tilted formation pose additional risk to shallow groundwater, partly due to the increase of lateral methane migration.
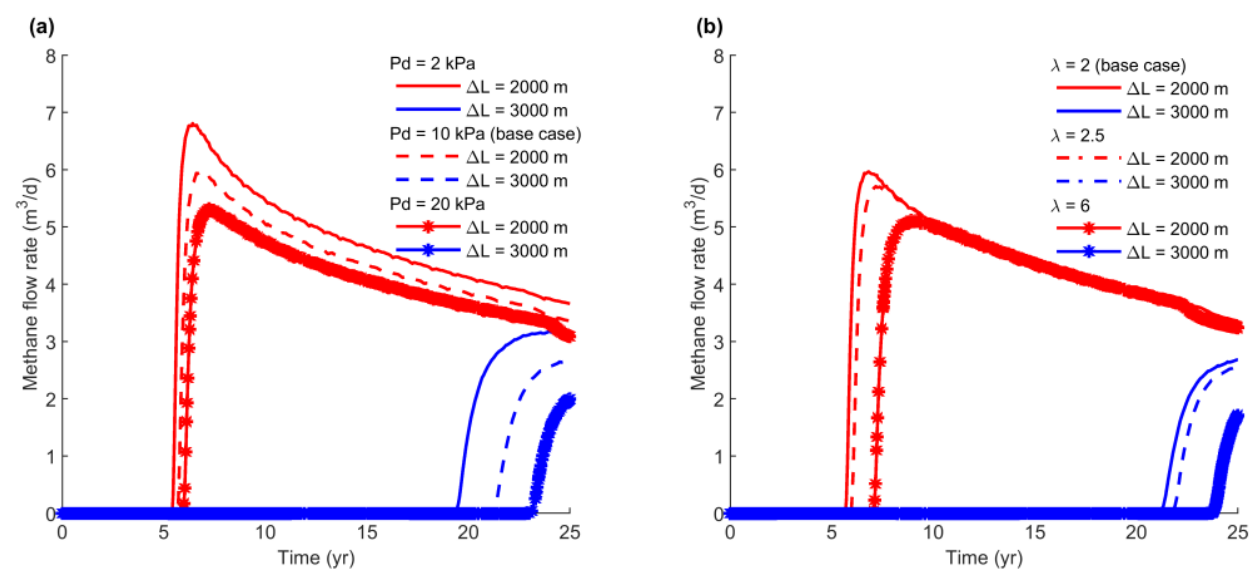
(c)
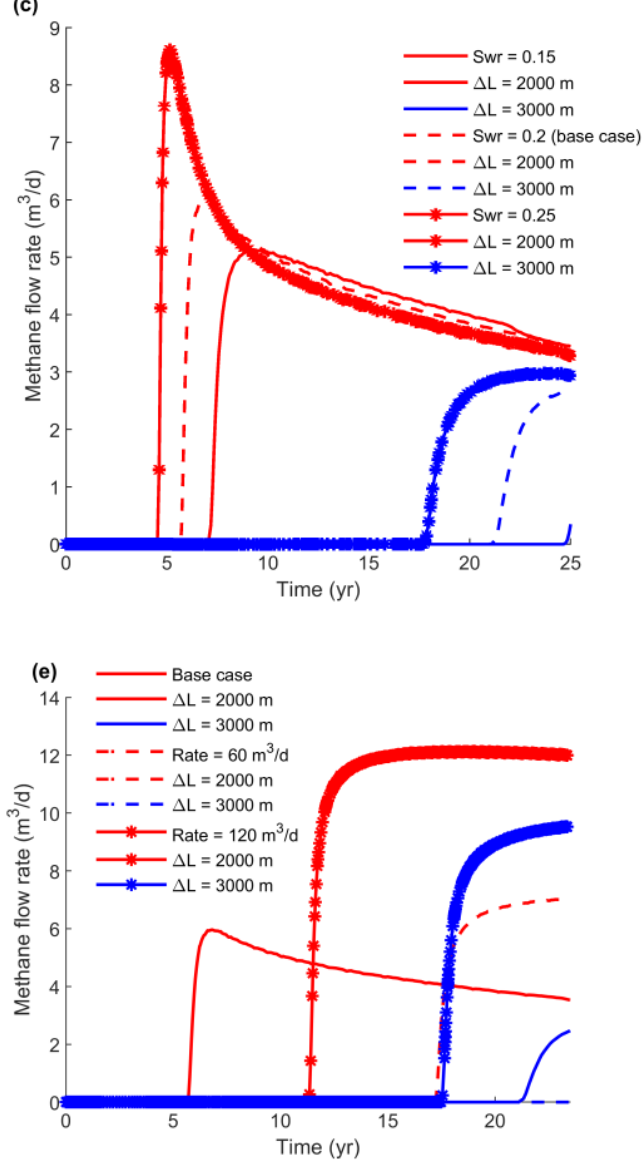
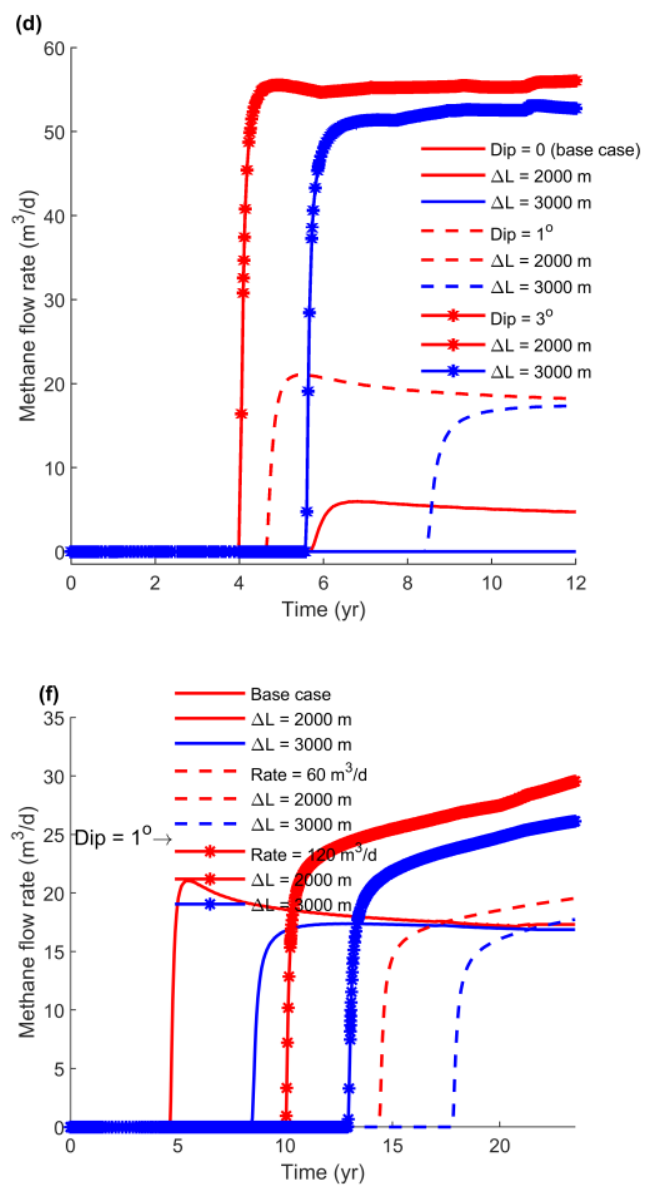

Figure 6-9. Methane flow rates at 2000 and $3000 \mathrm{~m}$ distances from the leaky gas well for the miscible flow model varying (a) entry pressure, (b) pore size distribution index, (c) residual water saturation, (d) tilt of formation, (e) methane inflow rate, and (f) methane inflow rate in a formation with $1^{\mathrm{o}}$ tilt.

\subsubsection{Claystone layer characteristics}

We assumed a discontinuity in the center of the claystone at the location $4500 \leq x \leq 5500 \mathrm{~m}$, to assess the influence of barrier layer integrity on the vertical movement of fluids. Methane migrates upward from the leakage source and accumulates at the claystone base. The trapped methane is compressed with the increase in hydrostatic pressure and tends to move upward through permeable pathways, e.g. fractures, and discontinuities. Once methane reaches the discontinuity, it preferentially migrates vertically along sediments with higher permeabilities (Figure 6-10a). The low pressure and temperature at shallow depths have strong impacts on the density of the free-phase gas and its solubility in the aqueous phase, subsequently fast vertical migration above the claystone formation slows the lateral spreading. Methane enters shallow groundwater after 2.4 years at a flow rate of $26.15 \mathrm{~m}^{3}$ day-1 and peaks at 3.3 years at a flow rate of $26.15 \mathrm{~m}^{3}$ day-1 $^{-1}$ (Figure 6-11). 
The impact of clay sequences is evaluated by considering two different field configurations (see the top panels in Figure 6-10(b-c)Figure 6-10). Each multi-layered claystone formation comprises four thin clay layers embedded in permeable sediments with random distribution, integrity and depth. In case I (Figure 6-10b), methane migrates upward until it is trapped by the claystone and spreads laterally in the direction of groundwater flow. The plume then flows upward from the left edge of the upper parts of the clay sequence because of the lack of an effective impermeable layer. Methane reaches the aquifer base after 4.8 years with a flow rate of $4.41 \mathrm{~m}^{3}$ day $^{-1}$. The plume evolution in case II (Figure 6-10c) is somewhat similar to case I. Methane migrates predominantly in the direction of groundwater flow, after being deviated by the claystone sequence. In this condition, viscous flow exceeds upward buoyancy. The methane plume arrives at the aquifer base after 3.7 years with a flow rate of $1.01 \mathrm{~m}^{3}$ day $^{-1}$.

As illustrated in Figure 6-11, continuous thin claystone layers constitute major safeguards against leakage of methane (free- and dissolved phase) to shallow aquifers. Time to breakthrough and methane flow rate to the aquifer depend on the integrity of the claystone and the positions of discontinuities with respect to the leaky gas well. The sequence of claystone layers strongly controls the temporal and spatial behavior of the plume in the subsurface and cumulative volume of methane reaching the shallow groundwater.

(a)

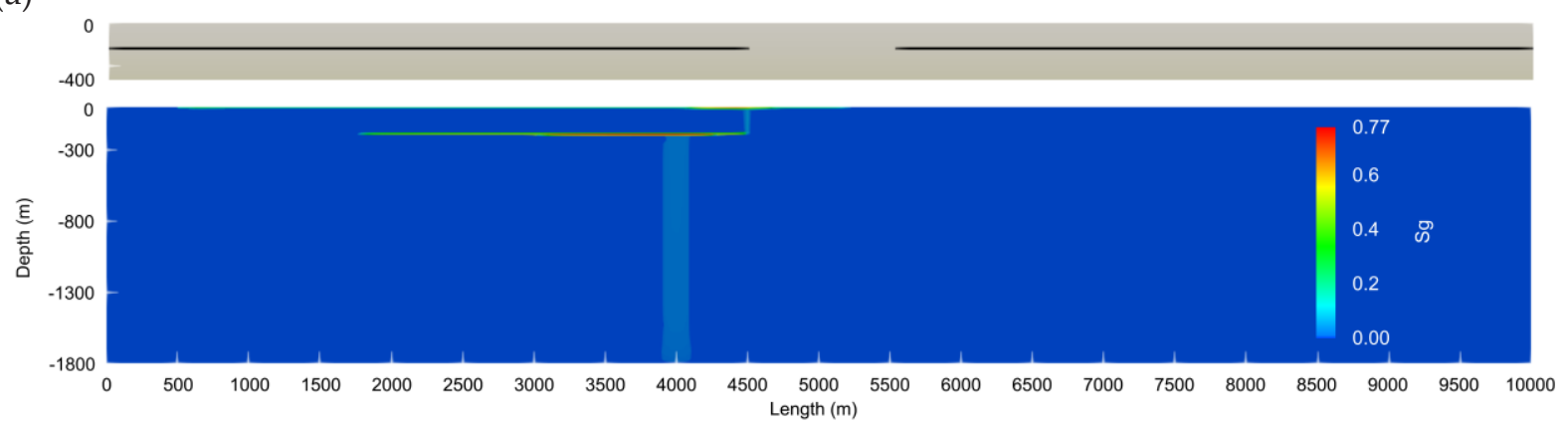

(b)

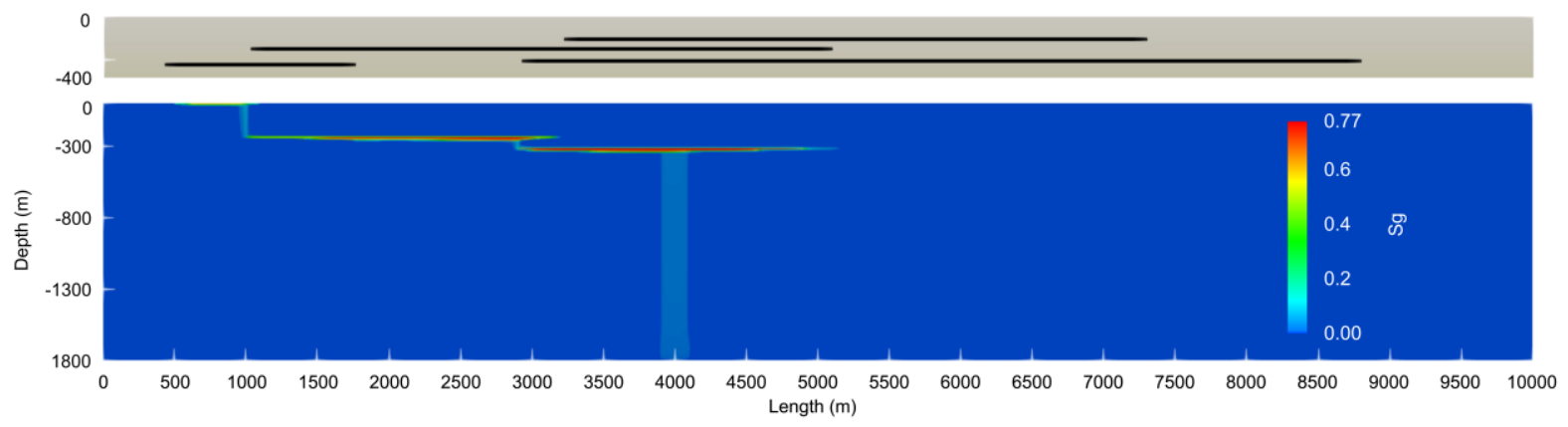


(c)

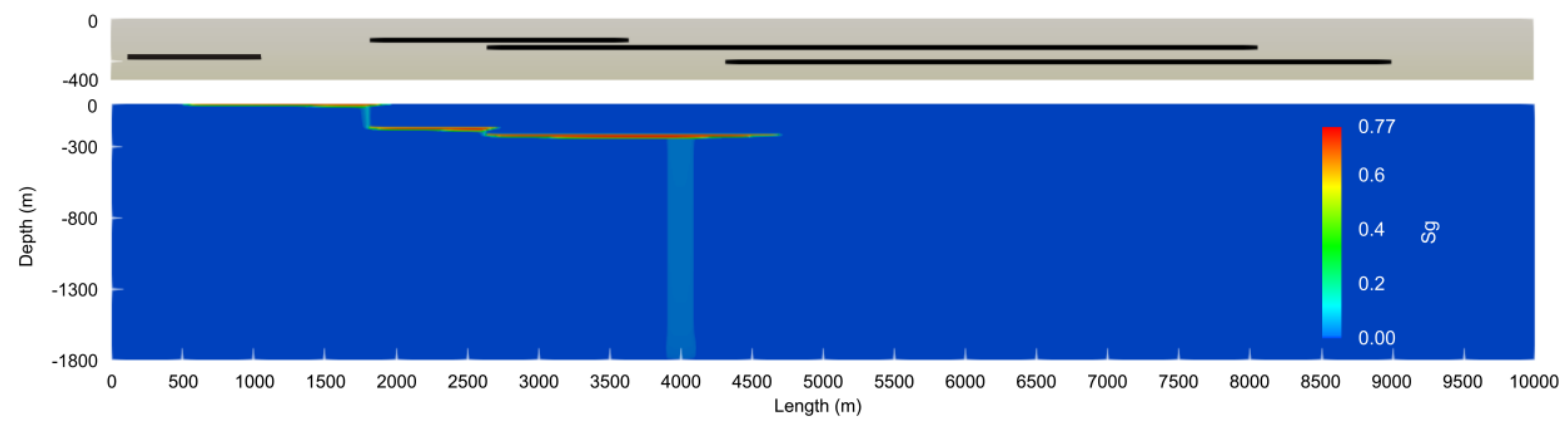

Figure 6-10. Gas phase saturation profiles after 15 years of methane leakage into the overburden. The influence of (a) clay layer integrity and different multi-layered clay systems ((b) case I, and (c) case II) are being analyzed. The top panels show the geometry of the clay layers at shallow depths.

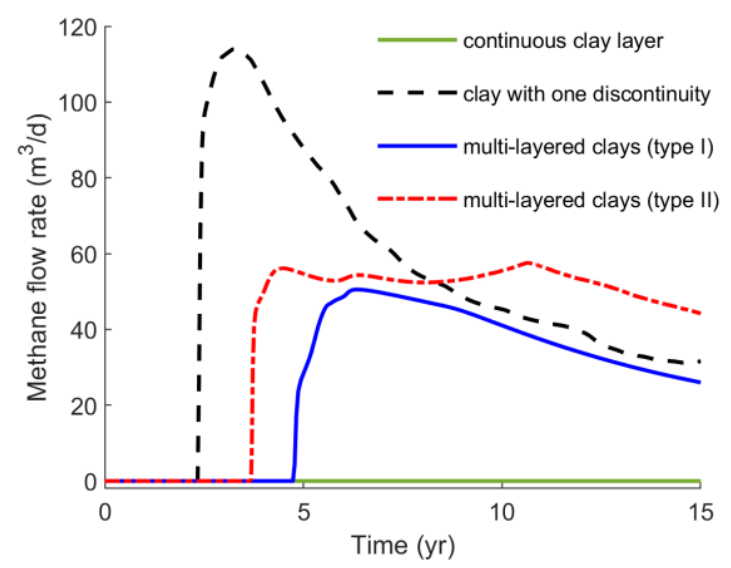

Figure 6-11. Measured methane flow into shallow groundwater for different geometries of clay layers.

\subsubsection{Comparison with observational studies}

The occurrence of thermogenic methane in groundwater may indicate that the gas has migrated upward from a hydrocarbon reservoir into the shallow subsurface. The presence of connective permeable pathways, e.g. faults, interconnected bedding planes and fractures, in the vicinity of the leaky gas well could result in an early manifestation of methane in groundwater wells (Cahill et al., 2017; Darrah et al., 2014; Gorody, 2012). For the leaked methane encountering low-permeability layers, methane preferentially flows along higher permeability sediments, either up dip (Figure 6-9) or in the direction of groundwater flow (Figure 6-10) (Steelman et al., 2017). For such conditions, methane can reach distances of hundreds of meters from the leaky well, delaying the breakthrough to the shallow aquifer system.

Elevated levels of methane concentrations have been observed in groundwater wells within approximately $1 \mathrm{~km}$ distance to hydrocarbon wells (Brantley et al., 2014; Darrah et al., 2014; 
Heilweil et al., 2015; Jackson et al., 2013; Osborn et al., 2011). Our results show that methane can be manifested even at distances of larger than $1 \mathrm{~km}$ from the leaky well because of the flow deviation along low-permeability rocks.

The temporal and spatial behavior of the methane plume in the subsurface highly depends on the geological and hydrogeological characteristics of the formations overlying the gas reservoir (Rice et al., 2018b; Steelman et al., 2017). The results indicate that methane can migrate to large distances from the source of leakage and thus the concentration of the methane in groundwater wells (if at all) can vary considerably with both time and space. Some observational studies (Li and Carlson, 2014; McMahon et al., 2017; Molofsky et al., 2013; Siegel et al., 2015) found no correlation between thermogenic methane in water samples from groundwater wells and just the distance to hydrocarbon wells corroborating our findings.

\subsection{Conclusions}

We built two-dimensional generic numerical models to assess hydrogeological conditions that could possibly lead groundwater contamination from the upward migration of methane from leaky natural gas wells. We applied miscible and immiscible multiphase flow models to evaluate the influence of methane solubility on the transport and examine the behavior of methane plume by means of each flow model. Furthermore, we conducted sensitivity analyses to investigate the relative importance of (1) hydrodynamic parameters including entry pressure and pore size distribution index in the Brooks-Corey equation, and residual water saturation, (2) methane leakage rate from the wellbore, (3) tilted formations, and (4) integrity and sequences of claystone layers. The use of relatively simple conceptual models allowed us to provide detailed evaluations on the influence of each parameter studied. Our simulations provide the following insights:

- Miscible and immiscible flow models result in a slightly different temporal and spatial behavior of the plume in the overburden due to the relatively low solubility of methane in the aqueous phase. Methane solubility has a small impact on the transport, especially at shallow depths.

- Gas leakage rate and leakage period are among the most important factors controlling the magnitude of methane migration to shallow depths. The persistent long-term 
leakage leads to the accumulation of large amounts of methane in the overburden, which could potentially be transported into the shallow aquifer system. A pulse of high methane flux is required to generate methane contamination in shallow groundwater for short times, for instance, methane arrival times at the measuring location at a distance of $2000 \mathrm{~m}$ from the leakage source were 11.3 and 5.7 years for continuous inflow rate of $120 \mathrm{~m}^{3} / \mathrm{d}$ and the base-case model, respectively. Results indicate that long-term methane leakage poses higher risks to groundwater in comparison to the rapid migration along fractures (Moortgat et al., 2018) and faults (Reagan et al., 2015) or during drilling operations (Zhang and Soeder, 2016).

- The buoyancy component of methane migration can be significant in formations/layers with a sufficient dip angle $\left(\geq 3^{\circ}\right)$ in horizontal and vertical direction. Under such conditions, the presence of low-permeability formations could further contribute to the horizontal spreading of the plume and methane can be observed in monitoring wells kilometers away from the leaky well. For example, instances of methane occurrence in groundwater reported in some of observational studies ( $\mathrm{Li}$ and Carlson, 2014; McMahon et al., 2017; Molofsky et al., 2013; Siegel et al., 2015) were not spatially correlated with locations of oil and gas wells, which could further confirm the migration of methane to different distances and directions from the gas well. Our simulations corroborate the results of previous studies that methane observation in groundwater is not concentrically aligned around the leakage source (Cahill et al., 2017; Jackson et al., 2013; Moortgat et al., 2018; Rice et al., 2018a; Steelman et al., 2017).

- In all modeled scenarios with a continuous claystone at shallow depth, the claystone constitutes an effective flow barrier. Simulations demonstrate that methane can be trapped at the base of a low-permeability unit and spread laterally until it reaches a discontinuity or a permeable vertically oriented pathway. Time to breakthrough and flow rates of methane to monitoring wells can vary strongly depending on the integrity, depth and distribution of low-permeability sediments with respect to the gas well. For example, in the scenario with a discontinuity in the center of the claystone (Figure 6-10), methane is observed in groundwater within $1 \mathrm{~km}$ radius around the well, while in scenarios with multiple claystone layers (Figure 6-10b and Figure 6-10c) methane is observed at a distance of more than $2 \mathrm{~km}$ from the gas well. Our simulations 
indicate that the complex shape of the methane plume in the subsurface, the arrival time to groundwater (in case of occurrence) and distances from a leaky gas well vary significantly based on hydrogeological characteristics of formations intercalated between aquifer and gas reservoir (Botner et al., 2018; Cahill et al., 2017; Darrah et al., 2014; Jackson et al., 2013; Osborn et al., 2011; Steelman et al., 2017). 


\section{Conclusions and Outlook}

\subsection{Conclusions}

The pollution of water resources is one of the primary concerns associated with hydraulic fracturing in unconventional gas development. Improved understanding of the fate and transport of contaminants in the subsurface helps to foster water quality monitoring programs aiming to reduce potential risks. Modelling of fluid migration from a deep gas formation into shallow groundwater is highly complex, due to complicated hydrogeological conditions, heterogeneity, multiphase system, etc. The appropriate use of conceptual models, applying an adequate degree of model simplifications that relates the nature of the data and predictions and verification of model results based on findings of observational studies, are the main challenges of numerical simulation of fluid migration.

This doctoral dissertation is built on a features, events, and processes (FEPs) database (Tatomir et al., 2015; Wiener et al., 2015) constructed within FracRisk project to evaluate groundwaterquality impacts of unconventional gas extraction. Chapter 2 provides a brief introduction to the FEP database and its applicability to direct modelling and model development for migration of fracturing and formation fluids in the subsurface from field to regional scales. Chapters 3 and 4 deal with the upward migration of fracturing fluid and brine along faults and abandoned wells during and after hydraulic fracturing. Chapter 5 deals with the development of a NAR neural network to predict leakage of fracturing fluid to the aquifer. Finally, in chapter 6, the transport of methane between a deep gas reservoir and shallow groundwater through overburden sediments is investigated. The main findings of this dissertation based upon numerical simulations can be summarized as follows: 


\subsubsection{Insights obtained from the FEPs database analysis (chapter 2)}

- The FEP analysis provides a framework for evaluating the performance and safety of fracturing operations and has the potential to be applied generically or to specific sites.

- FEP analysis facilitates the identification of scenarios describing potential situations that may lead to the contamination of groundwater, and the development of conceptual models to represent those failure scenarios. The conceptual models can be reviewed against the identified modelling requirements of each scenario and enhanced as required to ensure appropriate representation of the system.

- The structured, logical FEP approach presents the safety assessment of hydraulic fracturing in a clear and accessible manner, and facilitates the effective communication of information and opinion among all interested parties, e.g. scientists, stockholders and regulators.

\subsubsection{Insights obtained from fracturing fluid migration along a fault zone (chapter 3)}

- Most notably, fault properties and particularly fault permeability are the most influential factors controlling the flow rates of fracturing fluid reaching the aquifer. Fracturing injection pressure, overburden thickness and overburden hydraulic properties play important roles in restricting the upward flow of fluids. The rise of fracturing fluid to shallower strata is more sensitive to hydrogeological characteristics of the formations between the gas reservoir and the aquifer than the operational parameters.

- The combined influence of key parameters could hinder the upward flow of fracturing fluid. For instance, the failure scenario becomes more critical once the operation is conducted in shallow gas reservoirs (i.e. small vertical distances between the upper 
limit of hydraulic fractures and the aquifer) in the vicinity of a highly permeable pathway.

- The heterogeneity of overburden rocks tends to favor the horizontal spreading of fracturing fluid and limit the vertical propagation to the aquifer. The overburden heterogeneity could significantly reduce the potential risk to shallow groundwater.

- The modelling results corroborate findings of previous modelling studies (Birdsell et al., 2015a; Gassiat et al., 2013; Kissinger et al., 2013; Pfunt et al., 2016) including: (1) fracturing fluid does not migrate over a large vertical distance without the presence of a continuous vertical permeable pathway concocting the reservoir to the aquifer; (2) the probability of the aquifer contamination is low in a short-time period; and (3) the initial distribution of salinity in sedimentary basins could affect the upward fluid flow.

\subsubsection{Insights obtained from fracturing fluid migration along an abandoned well (chapter} 4)

- The abandoned well integrity is the most influential parameter on the migration of fracturing fluid. Properly sealed wellbores preclude the upward movement of fluids to overlying layers. The abandoned well distance to the operation could greatly affect the upward fluid flow. For instance, in the designed scenario, fracturing fluid cannot be detected in the aquifer when the distance of the well to the hydraulic fracture is 15 m.

- Well production extracts a significant amount of fracturing fluid (up to $67 \%$ of the injected volume) from the gas reservoir, thus limiting the probability of fracturing fluid flow to shallow depths. The combined effect of well production, and fracturing fluid 
dilution and mixing with formation water significantly reduces the likelihood and quantity of upward migration of fluids.

- The short-term probability of groundwater contamination is negligible, even in the presence of a leaky abandoned well. The overall contamination risk from fracturing fluid migration is low, i.e. $0.02 \%$ of fracturing fluid reached the aquifer over 30 years simulation period.

- This scenario corroborates many conclusions derived from the failure scenario investigating the leakage along a fault: (1) shut-in period has negligible influence on the upward flow of fracturing fluid; (2) hydraulic properties of the basin have more influence on the migration of fluids as compared to operational parameters; (3) the strata heterogeneity decreases the potential risk to the aquifer and (4) the probability of fracturing fluid upward movement is lower in basins with high salinity.

\subsubsection{Insights obtained from application of a NAR model to predict fracturing fluid leakage rate}

- The NAR network trained with Levenberg-Marquardt and Bayesian Regularization algorithms is able to predict fracturing fluid flow rate to the shallow aquifer with a sufficient accuracy.

- The prediction performance of NAR-BR model is slightly higher compared to NARLM model, especially when using a limited input dataset.

- NAR models can be applied as an additional effective approach for investigating the potential impacts of hydraulic fracturing on drinking water resources.

\subsubsection{Insights obtained from methane migration through overburden rocks (chapter 6)}

- Methane solubility has a small impact on the fluid transport, owing to the relatively low solubility of methane in the aqueous phase. 
- The spatial distribution of methane plume in the subsurface and the arrival time to shallow groundwater depends on the location and integrity of low permeability layers with respect to the source of leakage. Methane can be manifested at distances of larger than $1 \mathrm{~km}$ from the leaky well due to the flow deviation along low-permeability rocks and the presence of inclined features.

- The buoyancy component can be significant in formations/layers with a sufficient dip angle $\left(\geq 3^{\circ}\right)$. In such conditions, low-permeability layers could further contribute to the horizontal spreading of methane, thus the methane contamination is not concentric around the leakage source, and can vary significantly with both time and space.

- The rate and period of gas leakage are the key factors controlling the intensity level of methane migration. The persistent long-term gas inflow produces a large methane plume, which could spread vastly in different directions and travel along preferential pathways. The hydrogeological characteristics of the fracturing site, location and density of pre-existing pathways control the arrival time (if at all) and volume of methane reaching shallow groundwater.

\subsubsection{Main findings of this dissertation}

The probability of fracturing fluid and brine reaching shallow groundwater is low. A large volume of fracturing fluids is usually extracted during flowback and production periods and the remaining become diluted during the transport to shallow strata. The large vertical distance between the deep gas reservoir and shallow aquifers, low-permeability overburden layers and lack of a sufficient driving force, more often preclude the vertical extension of fracturing fluid. A limited volume of fracturing fluid can reach overlying aquifers if preferential pathways, e.g. faults and abandoned wells, present in the vicinity of fracturing 
operations. This holds true especially in shallow reservoirs or those with high permeable overburden layers. The influence of geological and hydrogeological characteristics of basins in the upward migration of fracturing fluid is substantial as compared to operational factors, especially in the long-term period.

The probability of methane reaching groundwater is much higher in comparison with fracturing fluid and deep brine, due to the strong buoyancy controlling the upward flow of free phase gas. Methane solubility in the aqueous phase is low, thus has a minimal influence on the transport. The presence of low permeability layers deviates methane flow, leading to the horizontal spread of the plume over large distances from the leaky natural gas well. The integrity and location of low permeability layers with respect to the gas well control the complex distribution of methane in the overburden, the arrival time (in case of occurrence) and accumulative concentration of methane reaching groundwater. Tilted features such as bedding planes and fractures further contribute to the lateral spreading of the plume, thus methane contamination is not concentric around the gas well and can differ significantly with both time and space. The finding shows that the design of long-term regular groundwater monitoring programs is necessary to ensure water quality remains intact.

\subsection{Outlook}

This dissertation has some shortcomings mainly resulting from the lack of information, and uncertainty in the parameter values. The demand for future development based on the preliminary findings of this dissertation can be summarized as follows:

- For the sakes of simplification, this study ignores heterogeneity in most of the modeled scenarios. The geological heterogeneity occurs at various scales and affects the extent 
of the contaminant plume and its arrival time to the aquifer. Implementing heterogeneous models by honoring real geological data is a complementary to the current work.

- The process of fluid migration to shallow groundwater is complex, thus, model validation and verification are challenging as analytical solutions and field measurement data are not available or not sufficient for providing confidence in the mathematical and numerical models. The benchmark problems provide a comparison between different modelling codes that use the same prescribed equations and input parameters. Benchmarking can help to explore the accuracy and reliability of numerical models. Future research could define benchmark problems in the frame of hydraulic fracturing, e.g., investigation of fracturing and formation fluids leakage through abandoned/active wellbores.

- This study can be further extend it by taking into account reactions, adsorption, and biological processes. Roy et al. (2016) concluded natural biodegradation could significantly mitigate methane concentrations in groundwater. The concentration of contaminants in groundwater can be attenuated over long time scales and needs to be further evaluated.

- One of the main sources of uncertainty in numerical simulations related to hydraulic fracturing is limited publicly available databases. The uncertainty of operational (e.g. gas leakage rate and period) and hydrogeological parameters (e.g. permeability of the aquifer and overburden layers) shall be considered in future studies for building risk assessment frameworks.

- Furfure effort may evaluate the frequency and impact of large methane leaks and its persistence or dissipation in groundwater over long-term periods. 
- Future research should further focus on the development of computational intelligence models such as neural networks to assess groundwater contamination impacts from unconventional gas development. 


\section{References}

Adamowski, J., Fung Chan, H., Prasher, S.O., Ozga-Zielinski, B., Sliusarieva, A., 2012. Comparison of multiple linear and nonlinear regression, autoregressive integrated moving average, artificial neural network, and wavelet artificial neural network methods for urban water demand forecasting in Montreal, Canada. Water Resour. Res. 48, W01528.

Aller, L., 1990. Handbook of suggested practices for the design and installation of groundwater monitoring wells. Vol. 1. Environmental Monitoring Systems Laboratory, Office of Research and Development, US Environmental Protection Agency.

Annevelink, M.P.J.A., Meesters, J.A.J., Hendriks, A.J., 2016. Environmental contamination due to shale gas development. Sci. Total Environ. 550, 431-438.

Arthur, J.D., Bohm, B., Coughlin, B.J., Layne, M., 2008. Hydraulic fracturing considerations for natural gas wells of the Fayetteville Shale. ALL Consult. 10.

Ayash, S.C., Dobroskok, A.A., Sorensen, J.A., Wolfe, S.L., Steadman, E.N., Harju, J.A., 2009. Probabilistic approach to evaluating seismicity in $\mathrm{CO} 2$ storage risk assessment. Energy Procedia 1, 2487-2494.

Bai, M., Reinicke, K., 2013. Numerical simulation of CO2 leakage through abandoned wells during $\mathrm{CO} 2$ underground storage. In: Clean Energy Systems in the Subsurface: Production, Storage and Conversion. Springer Berlin Heidelberg, pp. 197-210.

Barth-Naftilan, E., Aloysius, N., Saiers, J.E., 2015. Spatial and temporal trends in freshwater appropriation for natural gas development in Pennsylvania's Marcellus Shale Play. Geophys. Res. Lett. 42, 6348-6356.

Batzle, M., Wang, Z., 1992. Seismic properties of pore fluids. Geophysics 57, 1396-1408.

Bear, J., 2013. Dynamics of fluids in porous media. Courier Corporation.

Betz, D., Führer, F., Greiner, G., Plein, E., 1987. Evolution of the Lower Saxony basin. Tectonophysics 137, 127-170.

Birdsell, D.T., Rajaram, H., Dempsey, D., Viswanathan, H.S., 2015a. Hydraulic fracturing fluid migration in the subsurface: A review and expanded modeling results. Water Resour. 
Res. 51, 7159-7188.

Birdsell, D.T., Rajaram, H., Lackey, G., 2015b. Imbibition of hydraulic fracturing fluids into partially saturated shale. Water Resour. Res. 51, 6787-6796.

Bishop, C., 1995. Neural networks for pattern recognition. Oxford University Press.

Blair, B., Hughes, J., Allshouse, W., McKenzie, L., Adgate, J., Blair, B.D., Hughes, J., Allshouse, W.B., McKenzie, L.M., Adgate, J.L., 2018. Truck and multivehicle truck accidents with injuries observed near Colorado oil and gas operation. Int. J. Environ. Res. Public Health $15,1861$.

Botner, E.C., Townsend-Small, A., Nash, D.B., Xu, X., Schimmelmann, A., Miller, J.H., 2018. Monitoring concentration and isotopic composition of methane in groundwater in the Utica Shale hydraulic fracturing region of Ohio. Environ. Monit. Assess. 190, 322.

Bowers, G.L., 2002. Detecting high overpressure. Lead. Edge 21, 174-177.

Brantley, S.L., Yoxtheimer, D., Arjmand, S., Grieve, P., Vidic, R., Pollak, J., Llewellyn, G.T., Abad, J., Simon, C., 2014. Water resource impacts during unconventional shale gas development: The Pennsylvania experience. Int. J. Coal Geol. 126, 140-156.

Brooks, A.N., Corey, A.T., 1964. Hydraulic properties of porous media. Hydrol. Pap. Fort Collins, Color. State Univ.

Brown, S.P., Krupnick, A., Walls, M.A., 2009. Natural gas: a bridge to a low-carbon future. Issue Br. 09-11.

Brownlow, J.W., James, S.C., Yelderman, J.C., 2016. Influence of hydraulic fracturing on overlying aquifers in the presence of leaky abandoned wells. Groundwater 54, 781-792.

Brownlow, J.W., Yelderman, J.C., James, S.C., 2017. Spatial risk analysis of hydraulic fracturing near abandoned and converted oil and gas wells. Groundwater 55, 268-280.

Burden, F., Winkler, D., 2008. Bayesian regularization of neural networks. In: Artificial Neural Networks. Humana Press, pp. 23-42.

Butkovskyi, A., Bruning, H., Kools, S.A.E., Rijnaarts, H.H.M., Van Wezel, A.P., 2017. Organic pollutants in shale gas flowback and produced waters: identification, potential ecological 
impact, and implications for treatment strategies. Environ. Sci. Technol. 51, 4740-4754.

Cahill, A.G., Steelman, C.M., Forde, O., Kuloyo, O., Ruff, S.E., Mayer, B., Mayer, K.U., Strous, M., Ryan, M.C., Cherry, J.A., Parker, B.L., 2017. Mobility and persistence of methane in groundwater in a controlled-release field experiment. Nat. Geosci. 10, $289-294$.

Cao, V., Schaffer, M., Taherdangkoo, R., Licha, T., 2020. Solute reactive tracers for hydrogeological applications: A short review and future prospects. Water 12, 653.

Carman, P.C., 1937. Fluid flow through granular beds. Trans. Inst. Chem. Eng. 15, 150-166.

Carroll, S., Carey, J.W., Dzombak, D., Huerta, N.J., Li, L., Richard, T., Um, W., Walsh, S.D.C., Zhang, L., 2016. Review: Role of chemistry, mechanics, and transport on well integrity in CO2 storage environments. Int. J. Greenh. Gas Control 49, 149-160.

Celia, M.A., Bachu, S., Nordbotten, J.M., Gasda, S.E., Dahle, H.K., 2005. Quantitative estimation of CO2 leakage from geological storage: Analytical models, numerical models, and data needs. In: Greenhouse Gas Control Technologies 7. Elsevier Science Ltd, pp. $663-671$.

Chaudhary, A.S., Ehlig-Economides, C.A., Wattenbarger, R.A., 2011. Shale oil production performance from a stimulated reservoir volum. In: SPE Annual Technical Conference and Exhibition, 30 October-2 November. Denver, CO, USA.

Chen, J., Al-Wadei, M.H., Kennedy, R.C.M., Terry, P.D., 2014. Hydraulic fracturing: paving the way for a sustainable future? J. Environ. Public Health 2014, 656824.

Chen, S.S., Sun, Y., Tsang, D.C.W., Graham, N.J.D., Ok, Y.S., Feng, Y., Li, X.-D., 2017. Potential impact of flowback water from hydraulic fracturing on agricultural soil quality: Metal/metalloid bioaccessibility, Microtox bioassay, and enzyme activities. Sci. Total Environ. 579, 1419-1426.

Clancy, S.A., Worrall, F., Davies, R.J., Gluyas, J.G., 2018. The potential for spills and leaks of contaminated liquids from shale gas developments. Sci. Total Environ. 626, 1463-1473.

Cohen, H.A., Parratt, T., Andrews, C.B., 2013. Potential contaminant pathways from hydraulically fractured shale to aquifers (Letter). Ground Water 51, 317-319.

Cook, T., Perrin, J., Wagener, D.V., 2018. Hydraulically fractured horizontal wells account for 
most new oil and natural gas wells. US Energy Inf. Adm. Today Energy.

Da Costa Lopes, F., Watanabe, E.H., Rolim, L.G.B., 2015. A control-oriented model of a PEM fuel cell stack based on NARX and NOE neural networks. IEEE Trans. Ind. Electron. 62, $5155-5163$.

Darrah, T.H., Jackson, R.B., Vengosh, A., Warner, N.R., Whyte, C.J., Walsh, T.B., Kondash, A.J., Poreda, R.J., 2015. The evolution of Devonian hydrocarbon gases in shallow aquifers of the northern Appalachian Basin: Insights from integrating noble gas and hydrocarbon geochemistry. Geochim. Cosmochim. Acta 170, 321-355.

Darrah, T.H., Vengosh, A., Jackson, R.B., Warner, N.R., Poreda, R.J., Designed, R.J.P., Performed, R.J.P., 2014. Noble gases identify the mechanisms of fugitive gas contamination in drinking-water wells overlying the Marcellus and Barnett Shales. Proc. Natl. Acad. Sci. 111, 14076-14081.

Daubert, T.E., Danner, R.P., 1997. Physical and thermodynamic properties of pure chemicals: data compilation. Taylor \& Francis.

Dietrich, P., Helmig, R., Sauter, M., Hötzl, H., Köngeter, J., 2005. Flow and transport in fractured porous media. Springer Science \& Business Media.

Doan, C.D., Liong, S.Y., 2004. Generalization for multilayer neural network bayesian regularization or early stopping. In: Proceedings of Asia Pacific Association of Hydrology and Water Resources 2nd Conference. pp. 5-8.

Doornenbal, H., Stevenson, A., 2010. Petroleum Geological Atlas of the Southern Permian Basin Area, EAGE Publications b.v., Houten.

Duan, Z., Mao, S., 2006. A thermodynamic model for calculating methane solubility, density and gas phase composition of methane-bearing aqueous fluids from 273 to $523 \mathrm{~K}$ and from 1 to 2000 bar. Geochim. Cosmochim. Acta 70, 3369-3386.

Duan, Z., Møller, N., Greenberg, J., Weare, J.H., 1992a. The prediction of methane solubility in natural waters to high ionic strength from 0 to $250^{\circ} \mathrm{C}$ and from 0 to 1600 bar. Geochim. Cosmochim. Acta 56, 1451-1460.

Duan, Z., Møller, N., Weare, J.H., 1992b. An equation of state for the CH4-CO2-H2O system: 
I. Pure systems from 0 to $1000^{\circ} \mathrm{C}$ and 0 to 8000 bar. Geochim. Cosmochim. Acta 56, 26052617.

Duncan, I.J., 2015. Does methane pose significant health and public safety hazards? - A review. Environ. Geosci. 22, 85-96.

Dusseault, M., Jackson, R., 2014. Seepage pathway assessment for natural gas to shallow groundwater during well stimulation, in production, and after abandonment. Environ. Geosci. 21, 107-126.

Ebigbo, A., Class, H., Helmig, R., 2007. CO2 leakage through an abandoned well: Problemoriented benchmarks. Comput. Geosci. 11, 103-115.

Economides, M.J., Nolte, K.G., 1989. Reservoir stimulation (Vol. 2). Englewood Cliffs, NJ: Prentice Hall.

Edlmann, K., McDermott, C., 2016. D2.3 Hydro-geo-chemo-mechanical facies analysis relative to gas shales's of key basins.

Edwards, R.W.J., Celia, M.A., 2018. Shale gas well, hydraulic fracturing, and formation data to support modeling of gas and water flow in shale formations. Water Resour. Res. 54, 31963206.

Edwards, R.W.J., Doster, F., Celia, M.A., Bandilla, K.W., 2017. Numerical modeling of gas and water flow in shale gas formations with a focus on the fate of hydraulic fracturing fluid. Environ. Sci. Technol. 51, 13779-13787.

Entrekin, S., Trainor, A., Saiers, J., Patterson, L., Maloney, K., Fargione, J., Kiesecker, J., BaruchMordo, S., Konschnik, K., Wiseman, H., Nicot, J.P., 2018. Water Stress from High-Volume Hydraulic Fracturing Potentially Threatens Aquatic Biodiversity and Ecosystem Services in Arkansas, United States. Environ. Sci. Technol. 52, 2349-2358.

EPA, U., 2015. Review of state and industry spill data: characterization of hydraulic fracturingrelated spills. US Environmental Protection Agency Office of Research and Development, Washington, DC.

Eshleman, K.N., Elmore, A., 2013. Recommended best management practices for Marcellus shale gas development in Maryland. Appalach. Lab. Univ. Maryl. Cent. Environ. Sci. 
Frostburg, MD 21532.

Evans, D.J., 2009. A review of underground fuel storage events and putting risk into perspective with other areas of the energy supply chain. Geol. Soc. London, Spec. Publ. $313,173-216$.

Ferguson, G., McIntosh, J.C., Grasby, S.E., Hendry, M.J., Jasechko, S., Lindsay, M.B.J., Luijendijk, E., 2018. The persistence of brines in sedimentary basins. Geophys. Res. Lett. $45,4851-4858$.

Fisher, M., Warpinski, N., 2012. Hydraulic-fracture-height growth: Real data. SPE Prod. Oper. $27,8-19$.

Flemisch, B., Darcis, M., Erbertseder, K., Faigle, B., Lauser, A., Mosthaf, K., Müthing, S., Nuske, P., Tatomir, A., Wolff, M., Helmig, R., 2011. DuMux: DUNE for multi-\{phase, component, scale, physics, ... flow and transport in porous media. Adv. Water Resour. 34, 1102-1112.

Flewelling, S.A., Sharma, M., 2014. Constraints on upward migration of hydraulic fracturing fluid and brine. Groundwater 52, 9-19.

Flewelling, S.A., Tymchak, M.P., Warpinski, N., 2013. Hydraulic fracture height limits and fault interactions in tight oil and gas formations. Geophys. Res. Lett. 40, 3602-3606.

Foresee, F., Hagan, M.T., 1997. Gauss-Newton approximation to bayesian learning. In: Proceedings of the IEEE International Conference on Neural Networks-Conference Proceedings. Houston, Texas, pp. 1930-1935.

Freeze, R.A., Cherry, J.A., 1979. Groundwater. Prentice Hall, Eaglewood Cliffs, N. J.

Freyman, M., 2014. Hydraulic fracturing \& water stress: Water demand by the numbers. Ceres $85,49-50$.

Fuller, E.N., Schettler, P.D., Giddings, J.C., 1966. New method for prediction of binary gasphase diffusion coefficients. Ind. Eng. Chem. 58, 18-27.

Gasda, S.E., Bachu, S., Celia, M.A., 2004. Spatial characterization of the location of potentially leaky wells penetrating a deep saline aquifer in a mature sedimentary basin. Environ. Geol. 46, 707-720. 
Gasda, S.E., Celia, M.A., Wang, J.Z., Duguid, A., 2013. Wellbore permeability estimates from vertical interference testing of existing wells. Energy Procedia 37, 5673-5680.

Gasda, S.E., Nordbotten, J.M., Celia, M.A., 2008. Determining effective wellbore permeability from a field pressure test: A numerical analysis of detection limits. Environ. Geol. 54, $1207-1215$.

Gass, T.E., Lehr, J.H., Heiss, H.W., 1997. Impact of abandoned wells on ground water, US Robert S. Kerr Environmental Research Laboratory.

Gassiat, C., Gleeson, T., Lefebvre, R., McKenzie, J., 2013. Hydraulic fracturing in faulted sedimentary basins: Numerical simulation of potential contamination of shallow aquifers over long time scales. Water Resour. Res. 49, 8310-8327.

Ge, J., Ghassemi, A., 2011. Permeability enhancement in shale gas reservoirs after stimulation by hydraulic fracturing. In: 45th U.S. Rock Mechanics / Geomechanics Symposium, 26-29 June. American Rock Mechanics Association, San Francisco, California.

Gläser, D., Dell'Oca, A., Tatomir, A., Bensabat, J., Class, H., Guadagnini, A., Helmig, R., McDermott, C., Riva, M., Sauter, M., 2016. An approach towards a FEP-based model for risk assessment for hydraulic fracturing operations. Energy Procedia 97, 387-394.

Gleeson, T., Befus, K.M., Jasechko, S., Luijendijk, E., Cardenas, M.B., 2016. The global volume and distribution of modern groundwater. Nat. Geosci. 9, 161-167.

Gleeson, T., Smith, L., Moosdorf, N., Hartmann, J., Dürr, H.H., Manning, A.H., van Beek, L.P.H., Jellinek, A.M., 2011. Mapping permeability over the surface of the Earth. Geophys. Res. Lett. 38, L02401.

Goodman, P.S., Galatioto, F., Thorpe, N., Namdeo, A.K., Davies, R.J., Bird, R.N., 2016. Investigating the traffic-related environmental impacts of hydraulic-fracturing (fracking) operations. Environ. Int. 89-90, 248-260.

Gorody, A.W., 2012. Factors affecting the variability of stray gas concentration and composition in groundwater. Environ. Geosci. 19, 17-31.

Guzman, S.M., Paz, J.O., Tagert, M.L.M., 2017. The use of NARX neural networks to forecast daily groundwater levels. Water Resour. Manag. 31, 1591-1603. 
Hagan, M.T., Menhaj, M.B., 1994. Training feedforward networks with the Marquardt algorithm. IEEE Trans. Neural Netwrok 5, 989-993.

Harkness, J.S., Darrah, T.H., Warner, N.R., Whyte, C.J., Moore, M.T., Millot, R., Kloppmann, W., Jackson, R.B., Vengosh, A., 2017. The geochemistry of naturally occurring methane and saline groundwater in an area of unconventional shale gas development. Geochim. Cosmochim. Acta 208, 302-334.

Heilweil, V.M., Grieve, P.L., Hynek, S.A., Brantley, S.L., Solomon, D.K., Risser, D.W., 2015. Stream measurements locate thermogenic methane fluxes in groundwater discharge in an area of shale-gas development. Environ. Sci. Technol. 49, 4057-4065.

Helmig, R., 1997. Multiphase flow and transport processes in the subsurface: a contribution to the modeling of hydrosystems. Springer-Verlag.

Hendry, M.J., Schmeling, E.E., Barbour, S.L., Huang, M., Mundle, S.O.C., 2017. Fate and transport of shale-derived, biogenic methane. Sci. Rep. 7, 1-9.

IEA (International Energy Agency), 2018. World energy outlook 2018. Executive summary.

Jackson, R.B., Vengosh, A., Darrah, T.H., Warner, N.R., Down, A., Poreda, R.J., Osborn, S.G., Zhao, K., Karr, J.D., 2013. Increased stray gas abundance in a subset of drinking water wells near Marcellus shale gas extraction. Proc. Natl. Acad. Sci. 110, 11250-11255.

Kang, M., Baik, E., Miller, A.R., Bandilla, K.W., Celia, M.A., 2015. Effective permeabilities of abandoned oil and gas wells: Analysis of data from Pennsylvania. Environ. Sci. Technol. $49,4757-4764$.

Kekacs, D., Drollette, B.D., Brooker, M., Plata, D.L., Mouser, P.J., 2015. Aerobic biodegradation of organic compounds in hydraulic fracturing fluids. Biodegradation 26, 271-287.

Kelm, C.H., Faul, R.R., 1999. Well abandonment - A “best practices” approach can reduce environmental risk. In: SPE Asia Pacific Oil and Gas Conference and Exhibition, 20-22 April. Society of Petroleum Engineers, Jakarta, Indonesia.

King, G., 2012. Hydraulic fracturing 101: what every representative, environmentalist, regulator, reporter, investor, university researcher, neighbor and engineer should know about estimating frac risk and improving frac performance in unconventional gas and oil 
wells. In: SPE Hydraulic Fracturing Technology Conference, 6-8 February. The Woodlands, Texas, USA.

King, G.E., Rainbolt, M.F., Swanson, C., 2017. Frac hit induced production losses: evaluating root causes, damage location, possible prevention methods and success of remedial treatments. In: SPE Annual Technical Conference and Exhibition, 9-11 October. Society of Petroleum Engineers, San Antonio, Texas, USA.

Kisi, O., Cigizoglu, H.K., 2007. Comparison of different ANN techniques in river flow prediction. Civ. Eng. Environ. Syst. 24, 211-231.

Kissinger, A., Helmig, R., Ebigbo, A., Class, H., Lange, T., Sauter, M., Heitfeld, M., Klünker, J., Jahnke, W., 2013. Hydraulic fracturing in unconventional gas reservoirs: Risks in the geological system, part 2: Modelling the transport of fracturing fluids, brine and methane. Environ. Earth Sci. 70, 3855-3873.

Kissinger, A., Noack, V., Knopf, S., Konrad, W., Scheer, D., Class, H., 2017. Regional-scale brine migration along vertical pathways due to $\mathrm{CO} 2$ injection--Part 2: A simulated case study in the North German Basin. Hydrol. Earth Syst. Sci. 21, 2751-2775.

Kissinger, A., Noack, V., Knopf, S., Scheer, D., Konrad, W., Class, H., 2014. Characterization of reservoir conditions for $\mathrm{CO} 2$ storage using a dimensionless Gravitational Number applied to the North German Basin. Sustain. Energy Technol. Assessments 7, 209-220.

Klazinga, D.R., Steelman, C.M., Cahill, A.G., Walton, K.M., Endres, A.L., Parker, B.L., 2019. Methane gas transport in unconfined aquifers: A numerical sensitivity study of a controlled release experiment at CFB Borden. J. Contam. Hydrol. 225, 103506.

Kobayashi, K., 2004. Optimization methods for multiphase systems in the subsurface: application to methane migration in coal mining areas.

Kondash, A.J., Lauer, N.E., Vengosh, A., 2018. The intensification of the water footprint of hydraulic fracturing. Sci. Adv. 4, eaar5982.

Kozeny, J., 1927. Uber kapillare leitung der wasser in boden. R. Acad. Sci. Vienna, Proc. Cl. I $136271-306$.

Kreitler, C.W., 1989. Hydrogeology of sedimentary basins. J. Hydrol. 106, 29-53. 
Lacombe, S., Sudicky, E.A., Frape, S.K., Unger, A.J.A., 1995. Influence of leaky boreholes on cross-formational groundwater flow and contaminant transport. Water Resour. Res. 31, 1871-1882.

Lange, T., Sauter, M., Heitfeld, M., Schetelig, K., Brosig, K., Jahnke, W., Kissinger, A., Helmig, R., Ebigbo, A., Class, H., 2013. Hydraulic fracturing in unconventional gas reservoirs: risks in the geological system part 1. Environ. Earth Sci. 70, 3839-3853.

Lavrakas, P., 2008. Encyclopedia of survey research methods. Sage Publications.

Lawal, H., Jackson, G., Abolo, N., Flores, C., 2013. A novel approach to modeling and forecasting frac hits in shale gas wells. In: EAGE Annual Conference \& Exhibition Incorporating SPE Europec, 10-13 June. Society of Petroleum Engineers, London, UK.

Lehner, B., Verdin, K., Jarvis, A., 2008. New global hydrography derived from spaceborne elevation data. Eos, Trans. Am. Geophys. Union 89, 93-94.

Lewicki, J.L., Birkholzer, J., Tsang, C.F., 2007. Natural and industrial analogues for leakage of $\mathrm{CO} 2$ from storage reservoirs: identification of features, events, and processes and lessons learned. Environ. Geol. 52, 457-467.

Li, H., Carlson, K.H., 2014. Distribution and origin of groundwater methane in the Wattenberg oil and gas field of northern Colorado. Environ. Sci. Technol. 48, 1484-1491.

Li, Q., Xing, H., Liu, J., Liu, X., 2015. A review on hydraulic fracturing of unconventional reservoir. Petroleum 1, 8-15.

Ligtenberg, J.H., 2005. Detection of fluid migration pathways in seismic data: implications for fault seal analysis. Basin Res. 17, 141-153.

López, M., Valero, S., Senabre, C., Aparicio, J., Gabaldon, A., 2012. Application of SOM neural networks to short-term load forecasting: The Spanish electricity market case study. Electr. Power Syst. Res. 91, 18-27.

Loucks, D.P., Van Beek, E., Stedinger, J.R., Dijkman, J.P., Villars, M.T., 2005. Water resources systems planning and management: an introduction to methods, models, and applications. United Nations Educational, Scientific and Cultural Organization (UNESCO), Italy. 
Luijendijk, E., Gleeson, T., 2015. How well can we predict permeability in sedimentary basins? Deriving and evaluating porosity-permeability equations for noncemented sand and clay mixtures. Geofluids 15, 67-83.

MacKay, D.J.C., 1992a. Bayesian Interpolation. Neural Comput. 4, 415-447.

MacKay, D.J.C., 1992b. A Practical Bayesian Framework for Backpropagation Networks. Neural Comput. 4, 448-472.

Magri, F., Bayer, U., Jahnke, C., Clausnitzer, V., Diersch, H.J., Fuhrman, J., Möller, P., Pekdeger, A., Tesmer, M., Voigt, H.J., 2005. Fluid-dynamics driving saline water in the North East German Basin. Int. J. Earth Sci. 94, 1056-1069.

Maier, H.R., Dandy, G.C., 1996. The Use of Artificial Neural Networks for the Prediction of Water Quality Parameters. Water Resour. Res. 32, 1013-1022.

Maier, H.R., Dandy, G.C., 2000. Neural networks for the prediction and forecasting of water resources variables: A review of modelling issues and applications. Environ. Model. Softw. 15, 101-124.

Maitland, A., 2009. How many scale points should I include for attitudinal questions. Surv. Pract. 6.

Manceau, J.C., Tremosa, J., Audigane, P., Lerouge, C., Claret, F., Lettry, Y., Fierz, T., Nussbaum, C., 2015. Well integrity assessment under temperature and pressure stresses by a 1:1 scale wellbore experiment. Water Resour. Res. 51, 6093-6109.

Marquardt, D.W., 1963. An algorithm for least-squares estimation of nonlinear parameters. J. Soc. Ind. Appl. Math. 11, 431-441.

McDermott, C.I., Lodemann, M., Ghergut, I., Tenzer, H., Sauter, M., Kolditz, O., 2006. Investigation of coupled hydraulic-geomechanical processes at the KTB site: pressuredependent characteristics of a long-term pump test and elastic interpretation using a geomechanical facies model. Geofluids 6, 67-81.

McGlade, C., Speirs, J., Sorrell, S., 2013. Unconventional gas - A review of regional and global resource estimates. Energy 55, 571-584.

McLaughlin, M.C., Borch, T., Blotevogel, J., 2016. Spills of hydraulic fracturing chemicals on 
agricultural topsoil: biodegradation, sorption, and co-contaminant interactions. Environ. Sci. Technol. 50, 6071-6078.

McMahon, P.B., Barlow, J.R., Engle, M.A., Belitz, K., Ging, P.B., Hunt, A.G., Jurgens, B.C., Kharaka, Y.K., Tollett, R.W., Kresse, T.M., 2017. Methane and benzene in drinking-water wells overlying the Eagle Ford, Fayetteville, and Haynesville Shale hydrocarbon production areas. Environ. Sci. Technol. 51, 6727-6734.

Melchers, C., 2009. Methan im südlichen Münsterland: Genese, Migration und Gefahrenpotenzial. Dissertation, Westfälische Wilhelmsuniversität Münster, p 198.

Molofsky, L.J., Connor, J.A., Wylie, A.S., Wagner, T., Farhat, S.K., 2013. Evaluation of methane sources in groundwater in northeastern Pennsylvania. Groundwater 51, 333-349.

Montague, J.A., Pinder, G.F., 2015. Potential of hydraulically induced fractures to communicate with existing wellbores. Water Resour. Res. 51, 8303-8315.

Moortgat, J., Schwartz, F.W., Darrah, T.H., 2018. Numerical modeling of methane leakage from a faulty natural gas well into fractured tight formations. Groundwater 56, 163-175.

Morris, M.D., 1991. Factorial Sampling Plans for Preliminary Computational Experiments. Technometrics 33, 161-174.

Muehlenbachs, L., Krupnick, A.J., 2013. Shale gas development linked to traffic accidents in Pennsylvania. Common Resour. 3633-3659.

Myers, T., 2012a. Potential contaminant pathways from hydraulically fractured shale to aquifers. Groundwater 50, 872-882.

Myers, T., 2012b. Author's Reply. Ground Water 50, 828-830.

Neuzil, C.E., 1994. How permeable are clays and shales? Water Resour. Res. 30, 145-150.

Nicot, J.P., Mickler, P., Larson, T., Castro, M.C., Darvari, R., Uhlman, K., Costley, R., 2017. Methane occurrences in aquifers overlying the Barnett shale play with a focus on Parker County, Texas. Groundwater 55, 469-481.

Nicot, J.P., Scanlon, B.R., 2012. Water use for shale-gas production in Texas, US. Environ. Sci. Technol. 46, 3580-3586. 
Nogues, J.P., Nordbotten, J.M., Celia, M.A., 2011. Detecting leakage of brine or CO2 through abandoned wells in a geological sequestration operation using pressure monitoring wells. Energy Procedia 4, 3620-3627.

Nordbotten, J.M., Celia, M.A., Bachu, S., 2004. Analytical solutions for leakage rates through abandoned wells. Water Resour. Res. 40, W04204.

Nordbotten, J.M., Celia, M.A., Bachu, S., Dahle, H.K., 2005. Semianalytical solution for CO2 leakage through an abandoned well. Environ. Sci. Technol. 39, 602-611.

Nowamooz, A., Lemieux, J.M., Molson, J., Therrien, R., 2015. Numerical investigation of methane and formation fluid leakage along the casing of a decommissioned shale gas well. Water Resour. Res. 51, 4592-4622.

O’Donnell, M.C., Gilfillan, S.M. V., Edlmann, K., McDermott, C.I., 2018. Wastewater from hydraulic fracturing in the UK: assessing the viability and cost of management. Environ. Sci. Water Res. Technol. 4, 325-335.

Olyaie, E., Banejad, H., Chau, K.W., Melesse, A.M., 2015. A comparison of various artificial intelligence approaches performance for estimating suspended sediment load of river systems: a case study in United States. Environ. Monit. Assess. 187, 189.

Osborn, S.G., Vengosh, A., Warner, N.R., Jackson, R.B., 2011. Methane contamination of drinking water accompanying gas-well drilling and hydraulic fracturing. Proc. Natl. Acad. Sci. 108, 8172-8176.

Osborne, M.J., Swarbrick, R.E., 1997. Mechanisms for generating overpressure in sedimentary basins: a reevaluation. Am. Assoc. Pet. Geol. Bull. 81, 1023-1041.

Paulley, A., Metcalfe, R., Limer, L., 2011. Systematic FEP and scenario analysis to provide a framework for assessing long-term performance of the Krechba CO2 storage system at In Salah. Energy Procedia 4, 4185-4192.

Pawar, R.J., Watson, T.L., Gable, C.W., 2009. Numerical simulation of CO2 leakage through abandoned wells: model for an abandoned site with observed gas migration in Alberta, Canada. Energy Procedia 1, 3625-3632.

Peters, S.E., Husson, J.M., Czaplewski, J., 2018. Macrostrat: a platform for geological data 
integration and deep-time Earth crust research. Geochemistry, Geophys. Geosystems 19, 1393-1409.

Pfunt, H., Houben, G., Himmelsbach, T., 2016. Numerical modeling of fracking fluid migration through fault zones and fractures in the North German Basin. Hydrogeol. J. 24, 13431358.

Pitzer, K.S., Peiper, J.C., Busey, R.H., 1984. Thermodynamic properties of aqueous sodium chloride solutions. J. Phys. Chem. Ref. Data 13, 1-102.

Radonjic, M., Kupresan, D., 2014. Mechanical expansion of steel tubing as a solution to leaky wellbores. JoVE (Journal Vis. Exp. 93, e52098.

Reagan, M.T., Moridis, G.J., Keen, N.D., Johnson, J.N., 2015. Numerical simulation of the environmental impact of hydraulic fracturing of tight/shale gas reservoirs on near-surface groundwater: Background, base cases, shallow reservoirs, short-term gas, and water transport. Water Resour. Res. 4, 2543-2573.

Reid, R., Prausnitz, J., Poling, B., 1987. The properties of gases and liquids. McGraw-Hill: New York.

Reig, P., Luo, T., Proctor, J., 2014. Global shale gas development: water availability and business risks. World Resour. Inst.

Ren, G., Jiang, J., Younis, R.M., 2016. Fully coupled geomechanics and reservoir simulation for naturally and hydraulically fractured reservoirs. In: 50th U.S. Rock Mechanics/Geomechanics Symposium, 26-29 June. American Rock Mechanics Association, Houston, Texas.

Rice, A.K., Lackey, G., Proctor, J., Singha, K., 2018a. Groundwater-quality hazards of methane leakage from hydrocarbon wells: A review of observational and numerical studies and four testable hypotheses. Wiley Interdiscip. Rev. Water 5, e1283.

Rice, A.K., McCray, J.E., Singha, K., 2018b. Methane leakage from hydrocarbon wellbores into overlying groundwater: Numerical investigation of the multiphase flow processes governing migration. Water Resour. Res. 54, 2959-2975.

Rosa, L., Rulli, M.C., Davis, K.F., D’odorico, P., 2018. The water-energy nexus of hydraulic 
fracturing: a global hydrologic analysis for shale oil and gas extraction. Earth's Futur. 6, $745-756$.

Roy, N., Molson, J., Lemieux, J.M., Van Stempvoort, D., Nowamooz, A., 2016. Threedimensional numerical simulations of methane gas migration from decommissioned hydrocarbon production wells into shallow aquifers. Water Resour. Res. 52, 5598-5618.

Rozell, D.J., 2014. "Constraints on Upward Migration of Hydraulic Fracturing Fluid and Brine" by SA Flewelling and M. Sharma. Groundwater 52, 491-492.

Rudolph, T., Melchers, C., Minke, A., Coldewey, W.G., 2010. Gas seepages in Germany: Revisited subsurface permeabilities in the German mining district. Am. Assoc. Pet. Geol. Bull. 94, 847-867.

Ruiz, L., Cuéllar, M., Calvo-Flores, M., Jiménez, M., 2016. An application of non-linear autoregressive neural networks to predict energy consumption in public buildings. Energies 9, 684 .

Sahoo, S., Jha, M.K., 2013. Groundwater-level prediction using multiple linear regression and artificial neural network techniques: a comparative assessment. Hydrogeol. J. 21, 18651887.

Saiers, J.E., Barth, E., 2012. Potential contaminant pathways from hydraulically fractured shale to aquifers (Comment). Ground Water 50, 826-828.

Sauter, M., Brosig, K., Lange, T., Jahnke, W., Helmig, R., Kissinger, A., Heitfeld, M., Kluenker, J., Schetelig, K., 2012. Risiken im Geologischen System bei der Fracking-Technologie. Abschötzung der Auswirkungen auf Grundwasservorkommen.

Savage, D., Maul, P., Benbow, S., Walke, R., 2004. A Generic FEP Database for the Assessment of Long-Term Performance and Safety of the Geological Storage of CO2. Quintessa Rep. QRS-1060A-1.

Schout, G., Hartog, N., Hassanizadeh, S.M., Helmig, R., Griffioen, J., 2020. Impact of groundwater flow on methane gas migration and retention in unconsolidated aquifers. J. Contam. Hydrol. 230, 103619.

Schulz, R., Agemar, T., Alten, A.J., Kühne, K., Maul, A.A., Pester, S., Wirth, W., 2007. Aufbau 
eines geothermischen Informationssystems für Deutschland. Erdöl Erdgas Kohle 123, 7681.

Schwartz, M.O., 2015. Modelling the hypothetical methane-leakage in a shale-gas project and the impact on groundwater quality. Environ. Earth Sci. 73, 4619-4632.

Sclater, J.G., Christie, P.A.F., 1980. Continental stretching: An explanation of the Post-MidCretaceous subsidence of the central North Sea Basin. J. Geophys. Res. Solid Earth 85, $3711-3739$.

Shapiro, S.A., Dinske, C., Rothert, E., 2006. Hydraulic-fracturing controlled dynamics of microseismic clouds. Geophys. Res. Lett. 33, 1-5.

Sherwood, O.A., Rogers, J.D., Lackey, G., Burke, T.L., Osborn, S.G., Ryan, J.N., 2016. Groundwater methane in relation to oil and gas development and shallow coal seams in the Denver-Julesburg Basin of Colorado. Proc. Natl. Acad. Sci. 113, 8391-8396.

Shih, J.S., Saiers, J.E., Anisfeld, S.C., Chu, Z., Muehlenbachs, L.A., Olmstead, S.M., 2015. Characterization and Analysis of Liquid Waste from Marcellus Shale Gas Development. Environ. Sci. Technol. 49, 9557-9565.

Siegel, D.I., Azzolina, N.A., Smith, B.J., Perry, A.E., Bothun, R.L., 2015. Methane concentrations in water wells unrelated to proximity to existing oil and gas wells in northeastern Pennsylvania. Environ. Sci. Technol. 49, 4106-4112.

Soltanzadeh, H., Hawkes, C., 2009. Assessing fault reactivation tendency within and surrounding porous reservoirs during fluid production or injection. Int. J. Rock Mech. Min. Sci. 46, 1-7.

Steelman, C.M., Klazinga, D.R., Cahill, A.G., Endres, A.L., Parker, B.L., 2017. Monitoring the evolution and migration of a methane gas plume in an unconfined sandy aquifer using time-lapse GPR and ERT. J. Contam. Hydrol. 205, 12-24.

Sumi, L., 2008. Shale gas: Focus on the Marcellus shale. Oil Gas Account. Proj.

Sun, Y., Wang, D., Tsang, D.C.W., Wang, L., Ok, Y.S., Feng, Y., 2019. A critical review of risks, characteristics, and treatment strategies for potentially toxic elements in wastewater from shale gas extraction. Environ. Int. 125, 452-469. 
Taherdangkoo, R., Luijendijk, E., Potter van Loon, A., Gleeson, T., 2018. How clay layers control basin-scale fluid and heat flow. In: 20th EGU General Assembly, EGU2018, 4-13 April. Vienna, Austria, p. 12350.

Taherdangkoo, R., Tatomir, A., Anighoro, T., Sauter, M., 2019. Modeling fate and transport of hydraulic fracturing fluid in the presence of abandoned wells. J. Contam. Hydrol. 221, $58-68$.

Taherdangkoo, R., Tatomir, A., Taylor, R., Sauter, M., 2017. Numerical investigations of upward migration of fracking fluid along a fault zone during and after stimulation. Energy Procedia 125, 126-135.

Tatomir, A., McDermott, C., Bensabat, J., Class, H., Edlmann, K., Taherdangkoo, R., Sauter, M., 2018. Conceptual model development using a generic Features, Events, and Processes (FEP) database for assessing the potential impact of hydraulic fracturing on groundwater aquifers. Adv. Geosci. 45, 185-192.

Tatomir, A., McDermott, C., Edlmann, K., Bensabat, J., Wiener, H., Goren, Y., 2015. D3.1 Update of FEP database (Version 2).

Tatomir, A., Sauter, M., Taherdangkoo, R., Bensabat, J., Wiener, H., McDermott, C., Edlmann, K., Gläser, D., Class, H., Helmig, R., Figueiredo, B., Hedayati, M., Niemi, A., Tsan, C.F., Carrera, J., Maier, U., 2016. D3.2 Characterization of the Key FEP risk scenarios.

Tesmer, M., Möller, P., Wieland, S., Jahnke, C., Voigt, H., Pekdeger, A., 2007. Deep reaching fluid flow in the North East German Basin: origin and processes of groundwater salinisation. Hydrogeol. J. 15, 1291-1306.

U.S. EPA, 2015. Assessment of the potential impacts of hydraulic fracturing for oil and gas on drinking water resources (external review draft). U.S. EPA: Washington, DC.

US EIA, 2011a. Review of emerging resources: U.S. shale gas and shale oil plays. U.S. Dep. of Energy, Washington, D. C.

US EIA, 2011b. World shale gas resources: an initial assessment of 14 regions outside the United States. U.S. Dep. of Energy, Washington, D. C.

Vengosh, A., Jackson, R., Warner, N., 2014. A critical review of the risks to water resources 
from unconventional shale gas development and hydraulic fracturing in the United States. Environ. Sci. Technol. 48, 8334-8348.

Walke, R., Metcalfe, R., Limer, L., Maul, P., Paulley, A., Savage, D., 2011. Experience of the application of a database of generic Features, Events and Processes (FEPs) targeted at geological storage of CO2. Energy Procedia 4, 4059-4066.

Walter, R., 2007. Geologie von mitteleuropa. Geschiebekd. Aktuell 23, S 122.

Wang, K.W., Deng, C., Li, J.P., Zhang, Y.Y., Li, X.Y., Wu, M.C., 2017. Hybrid methodology for tuberculosis incidence time-series forecasting based on ARIMA and a NAR neural network. Epidemiol. Infect. 145, 1118-1129.

Wang, L., Torres, A., Xiang, L., Fei, X., Naido, A., Wu, W., 2015. A technical review on shale gas production and unconventional reservoirs modeling. Nat. Resour. 6, 141-151.

Wang, Y., Li, X., Tang, C.A., 2016. Effect of injection rate on hydraulic fracturing in naturally fractured shale formations: a numerical study. Environ. Earth Sci. 75, 935.

Warner, N.R., Jackson, R.B., Darrah, T.H., Osborn, S.G., Down, A., Zhao, K., White, A., Vengosh, A., 2012. Geochemical evidence for possible natural migration of Marcellus Formation brine to shallow aquifers in Pennsylvania. Proc. Natl. Acad. Sci. 109, 1196111966.

Wei, S., Zuo, D., Song, J., 2012. Improving prediction accuracy of river discharge time series using a Wavelet-NAR artificial neural network. J. Hydroinformatics 14, 974-991.

Weibel, R., Kristensen, L., Olivarius, M., Leth Hjuler, M., Mathiesen, A., Nielsen, L.H., 2012. Investigating deviations from overall porosity-permeability trends. In: Proceedings 36th Workshop on Geothermal Reservoir Engineering, Stanford University, California (Vol. 16).

Wen, T., Castro, M.C., Nicot, J.P., Hall, C.M., Larson, T., Mickler, P., Darvari, R., 2016. Methane sources and migration mechanisms in shallow groundwaters in Parker and Hood Counties, Texas-A heavy noble gas analysis. Environ. Sci. Technol. 50, 12012-12021.

Weng, X., Kresse, O., Cohen, C.E., Wu, R., Gu, H., 2011. Modeling of hydraulic fracture network propagation in a naturally fractured formation. In: SPE Hydraulic Fracturing 
Technology Conference, 24-26 January. Society of Petroleum Engineers, The Woodlands, Texas, USA.

Wiener, H., Goren, Y., Bensabat, J., Tatomir, A., Edlmann, K., McDermott, C., 2015. D4.1 Ranked FEP list.

Wilson, M.P., Worrall, F., Davies, R.J., Hart, A., 2017. Shallow aquifer vulnerability from subsurface fluid injection at a proposed shale gas hydraulic fracturing site. Water Resour. Res. 53, 9922-9940.

Wolfgramm, M., Seibt, A., 2008. Zusammensetzung von Tiefenwässern in Deutschland und ihre Relevanz für geothermische Anlagen, GtV-Tagung in Karlsruhe 2008. Proceedings.

Wolfgramm, M., Thorwart, K., Rauppach, K., Brandes, J., 2011. Zusammensetzung, Herkunft und Genese geothermaler Tiefengrundwässer im Norddeutschen Becken (NDB) und deren Relevanz für die geothermische Nutzung. Z geol Wiss 339, 173-193.

Yavuz, F., van Tilburg, T., David, P., Spruijt, M., Wildenborg, T., 2009. Second Generation CO2 FEP Analysis: CASSIF - Carbon Storage Scenario Identification Framework. Energy Procedia 1, 2479-2485.

Yu, G., Aguilera, R., 2012. 3D analytical modeling of hydraulic fracturing stimulated reservoir volume. In: SPE Latin America and Caribbean Petroleum Engineering Conference, 16-18 April. Mexico City, Mexico.

Zhang, D., Yang, T., 2015. Environmental impacts of hydraulic fracturing in shale gas development in the United States. Pet. Explor. Dev. 42, 876-883.

Zhang, L., Soeder, D.J., 2016. Modeling of methane migration in shallow aquifers from shale gas well drilling. Groundwater 54, 345-353.

Zhang, Q., Stanley, S.J., 1997. Forecasting raw-water quality parameters for the north Saskatchewan river by neural network modeling. Water Res. 31, 2340-2350.

Zheltov, A., 1955. 3. Formation of vertical fractures by means of highly viscous liquid. In: 4th World Petroleum Congress, 6-15 June. World Petroleum Congress, Rome, Italy.

Zhu, Y., Xu, S., Payne, M., Martinez, A., Liu, E., Harris, C., Bandyopadhyay, K., 2012. Improved rock-physics model for shale gas reservoirs. In: SEG Annual Meeting, 4-9 November. 
Society of Exploration Geophysicists, Las Vegas, Nevada.

Ziegler, P.A., 1990. Geological atlas of western and central Europe. Geol. Soc. London. 
